\title{
Algebraic actions of discrete groups: the $p$-adic method
}

by

\author{
Serge Cantat \\ Université de Rennes 1 \\ Rennes, France
}

\author{
JUNYI XIE \\ Université de Rennes 1 \\ Rennes, France
}

\section{Contents}

1. Introduction . . . . . . . . . . . . . . . . . . . . . 239

2. Tate analytic diffeomorphisms of the $p$-adic polydisk . . . . . . . 244

3. Good models, $p$-adic integers, and invariant polydisks . . . . . . 253

4. Regular actions of $\mathrm{SL}_{n}(\mathbf{Z})$ on quasi-projective varieties . . . . . . . 258

5. Actions of $\mathrm{SL}_{n}(\mathbf{Z})$ in dimension $n-1 \ldots \ldots \ldots \ldots 26 \ldots$

6. Mapping class groups and nilpotent groups . . . . . . . . . . . 269

7. Periodic orbits and invariant polydisks . . . . . . . . . 273

8. Birational actions of lattices on quasi-projective varieties . . . . . 285

9. Appendix . . . . . . . . . . . . . . . . . . . 289

References . . . . . . . . . . . . . . . . . . . . 292

\section{Introduction}

\subsection{Automorphisms and birational transformations}

Let $X$ be a quasi-projective variety of dimension $d$, defined over the field of complex numbers. Let $\operatorname{Aut}(X)$ denote its group of (regular) automorphisms and $\operatorname{Bir}(X)$ its group of birational transformations. A good example is provided by the affine space $\mathbb{A}_{\mathbf{C}}^{d}$ of dimension $d \geqslant 2$ : Its group of automorphisms is "infinite-dimensional" and contains elements with a rich dynamical behavior (see [34], [3]); its group of birational transformations is the Cremona group $\mathrm{Cr}_{d}(\mathbf{C})$, and is known to be much larger than Aut $\left(\mathbb{A}_{\mathbf{C}}^{d}\right)$.

We present two new arguments that can be combined to study finitely generated groups acting by automorphisms or birational transformations. They lead to new constraints on groups of birational transformations, in any dimension. 
The first argument is based on $p$-adic analysis and may be viewed as an extension of two classical strategies from a linear to a non-linear context. The first strategy appeared in the proof of the theorem of Skolem, Mahler, and Lech, which says that the zeros of a linear recurrence sequence occur along a finite union of arithmetic progressions. This method plays now a central role in arithmetic dynamics (see [6], [7], [55]). The second strategy has been developed by Bass, Milnor, and Serre when they obtained rigidity results for finite-dimensional linear representations of $\mathrm{SL}_{n}(\mathbf{Z})$ as a corollary of the congruence subgroup property (see [2], [62]). Here, we combine these strategies for non-linear actions of finitely generated groups of birational transformations.

Our second argument makes use of isoperimetric inequalities from geometric group theory and of the Lang-Weil estimates from diophantine geometry. We now list the main results that follow from the combination of those arguments.

\subsection{Actions of $\mathrm{SL}_{n}(\mathrm{Z})$}

Consider the group $\mathrm{SL}_{n}(\mathbf{Z})$ of $n \times n$ matrices with integer entries and determinant 1 . Let $\Gamma$ be a finite-index subgroup of $\mathrm{SL}_{n}(\mathbf{Z})$; it acts by linear projective transformations on the projective space $\mathbb{P}_{\mathbf{C}}^{n-1}$, and the kernel of the homomorphism $\Gamma \rightarrow \mathrm{PGL}_{n}(\mathbf{C})$ contains at most two elements. The following result shows that $\Gamma$ does not act faithfully on any smaller variety.

Theorem A. Let $\Gamma$ be a finite-index subgroup of $\mathrm{SL}_{n}(\mathbf{Z})$. Let $X$ be an irreducible, complex, quasi-projective variety. If $\Gamma$ embeds into $\operatorname{Aut}(X)$, then $\operatorname{dim}_{\mathbf{C}}(X) \geqslant n-1$. If $\operatorname{dim}_{\mathbf{C}}(X)=n-1$, then there is an isomorphism $\tau: X \rightarrow \mathbb{P}_{\mathbf{C}}^{n-1}$ which conjugates the action of $\Gamma$ on $X$ to a linear projective action on $\mathbb{P}_{\mathbf{C}}^{n-1}$.

Let $\mathbf{k}$ and $\mathbf{k}^{\prime}$ be fields of characteristic zero. Every field of characteristic zero which is generated by finitely many elements embeds into C. Since finite-index subgroups of $\mathrm{SL}_{n}(\mathbf{Z})$ are finitely generated, Theorem A implies: (1) the group $\mathrm{SL}_{n}(\mathbf{Z})$ embeds into $\operatorname{Aut}\left(\mathbb{A}_{\mathbf{k}}^{d}\right)$ if and only if $d \geqslant n ;(2)$ if $\operatorname{Aut}\left(\mathbb{A}_{\mathbf{k}}^{d}\right)$ embeds into Aut $\left(\mathbb{A}_{\mathbf{k}^{\prime}}^{d^{\prime}}\right)$ (as abstract groups), then $d \leqslant d^{\prime}$. Previous proofs of assertion (2) assumed $\mathbf{k}$ to be equal to $\mathbf{C}$ (see [27], [45]).

\subsection{Lattices in simple Lie groups}

Theorem A may be extended in two directions, replacing $\mathrm{SL}_{n}(\mathbf{Z})$ by more general lattices, and looking at actions by birational transformations instead of automorphisms. Let $\mathbf{S}$ be an absolutely almost simple linear algebraic group which is defined over $\mathbf{Q}$; fix an embedding of $\mathbf{S}$ in $\mathrm{GL}_{n}$ (over $\mathbf{Q}$ ). The $\mathbf{Q}$-rank of $\mathbf{S}$ is the maximal dimension of a Zariski-closed subgroup of $\mathbf{S}$ that is diagonalizable over $\mathbf{Q}$; the $\mathbf{R}$-rank of $\mathbf{S}$ is the maximal 
dimension of a Zariski-closed subgroup that is diagonalizable over $\mathbf{R}$. The subgroup $\mathbf{S}(\mathbf{Z})$ is a lattice in $\mathbf{S}(\mathbf{R})$; it is cocompact if and only if the $\mathbf{Q}$-rank of $\mathbf{S}$ vanishes (see [8]).

TheOrem B. Let $X$ be an irreducible complex projective variety. Let $\mathrm{S} \mathrm{GL}_{n}$ be a linear algebraic group, over the field of rational numbers $\mathbf{Q}$. Assume that $\mathbf{S}$ is absolutely almost simple, and that the lattice $\mathbf{S}(\mathbf{Z})$ is not cocompact in $\mathbf{S}(\mathbf{R})$. If a finite-index subgroup of $\mathrm{S}(\mathbf{Z})$ embeds into $\operatorname{Bir}(X)$, then

$$
\operatorname{dim}_{\mathbf{C}}(X) \geqslant \operatorname{rank}_{\mathbf{R}}(S)
$$

If $\operatorname{dim}(X)=\operatorname{rank}_{\mathbf{R}}(\mathrm{S}) \geqslant 2$, then $\mathbf{S}_{\mathbf{R}}$ is $\mathbf{R}$-isogeneous to $\mathrm{SL}_{\operatorname{dim}(X)+1, \mathbf{R}}$.

As a corollary, the Cremona group $\mathrm{Cr}_{d}(\mathbf{k})$ does not embed into $\mathrm{Cr}_{d^{\prime}}\left(\mathbf{k}^{\prime}\right)$ if $\mathbf{k}$ and $\mathbf{k}^{\prime}$ have characteristic zero and $d>d^{\prime}$.

Remark 1.1. If $\operatorname{rank}_{\mathbf{R}}(S) \geqslant 2$, every lattice $\Gamma$ of $\mathbf{S}(\mathbf{R})$ is almost simple: Its normal subgroups are finite and central, or cofinite (see $\S 4.2$ and 8.1). Thus, the assumption " $\Gamma$ embeds into $\operatorname{Bir}(X)$ " can be replaced by "there is a homomorphism from $\Gamma$ to $\operatorname{Bir}(X)$ with infinite image". Using the Margulis arithmeticity theorem, one can replace $\mathrm{S}$ by any simple real Lie group $H$ with $\operatorname{rank}_{\mathbf{R}}(H) \geqslant 2$, and $\mathbf{S}(\mathbf{Z})$ by any non-uniform lattice of $H$ in the statement of Theorem B.

Remark 1.2. The statement of Theorem B concerns non-uniform lattices, because the proof makes use of the congruence subgroup property, and the congruence kernel is known to be finite for all those lattices. There are also uniform lattices for which this property is known and the same proof applies (for instance for all lattices in Qanisotropic spin groups for quadratic forms in $m \geqslant 5$ variables with real rank $\geqslant 2$; see [43], [64]).

Remark 1.3. The main theorems of [11], [19] extend Theorem B to all types of lattices (including cocompact lattices) in simple real Lie groups, but assume that the action is by regular automorphisms on a compact Kähler manifold. When $X$ is compact, $\operatorname{Aut}(X)$ is a complex Lie group: It may have infinitely many connected components, but its dimension is finite. The techniques of [11], [19] do not apply to arbitrary quasiprojective varieties (for instance to $X=\mathbb{A}_{\mathbf{C}}^{d}$ ) and to groups of birational transformations. See [17], [13], [26] for groups of birational transformations of surfaces.

Example 1.4. There is a lattice in $\mathrm{SO}_{1,9}(\mathbf{R})$ which acts faithfully on a rational surface by regular automorphisms: This is due to Coble (see $[16, \S 3.4]$ ). A similar phenomenon holds for general Enriques surfaces (see [23]). Thus, lattices in simple Lie groups of large dimension may act faithfully on small-dimensional varieties. 


\subsection{Finite fields and Hrushovski's theorem}

Let $\Gamma$ be a finite-index subgroup of $\mathbf{S}(\mathbf{Z})$, where $\mathbf{S}$ is as in Theorem B. To prove Theorems $\mathrm{A}$ and $\mathrm{B}$, we first change the field of definition, replacing $\mathbf{C}$ by the field of $p$-adic numbers $\mathbf{Q}_{p}$ for some large prime number $p$; indeed, since the ring generated by the coefficients of the formulae defining the variety $X$ and the generators of the group $\Gamma \subset \operatorname{Bir}(X)$ is finitely generated, we may embed this ring in $\mathbf{Z}_{p}$ for some large prime $p$.

Then, we prove that there exists a finite extension $K$ of $\mathbf{Q}_{p}$ and a $p$-adic polydisk in $X(K)$ which is invariant under the action of a finite-index subgroup $\Gamma^{\prime}$ of $\Gamma$, and on which $\Gamma^{\prime}$ acts by Tate analytic diffeomorphisms. Those polydisks correspond to periodic orbits for the action of $\Gamma$ on $X(\mathbf{F})$, where $\mathbf{F}$ is the residue field of the non-archimedean field $K$. Thus, an important step toward Theorem B is the existence of pairs $\left(m, \Gamma^{\prime}\right)$, where $m$ is in $X(\mathbf{F}), \Gamma^{\prime}$ is a finite-index subgroup of $\Gamma$, and all elements of $\Gamma^{\prime}$ are well defined at $m$ and fix $m$ (no element of $\Gamma^{\prime}$ has an indeterminacy at $m$ ). For cyclic groups of transformations, this follows from a theorem of Hrushovski (see [41]). Here, we combine the Lang-Weil estimates with isoperimetric inequalities from geometric group theory: The existence of the pair $\left(m, \Gamma^{\prime}\right)$ is obtained for groups with Kazhdan property $(\mathrm{T})$ in Theorems 7.10 and 7.13; the argument applies also to other types of groups (see $\$ 7.2 .5$ ).

Once such invariant polydisks are constructed, several corollaries easily follow (see $\S 7.4 .2)$. For instance, we get the following result.

THEOREM C. If a discrete group $\Lambda$ with Kazhdan property (T) acts faithfully by birational transformations on a complex projective variety $X$, the group $\Lambda$ is residually finite and contains a torsion-free, finite-index subgroup.

\subsection{The $p$-adic method}

When an invariant $p$-adic polydisk is constructed, a theorem of Bell and Poonen provides a tool to extend the action of every element $\gamma$ in our group into a Tate analytic action of the additive group $\mathbf{Z}_{p}$. When $\Gamma$ has finite index in $\mathbf{S}(\mathbf{Z})$, as in Theorem $\mathrm{B}$, and $\operatorname{rank}_{\mathbf{R}}(S) \geqslant 2$, this may be combined with the congruence subgroup property: We prove that the action of the lattice extends to an action of a finite-index subgroup of the $p$-adic group $\mathbf{S}\left(\mathbf{Z}_{p}\right)$ by Tate analytic transformations (Theorem 2.11). Thus, starting with a countable group of birational transformations, we end up with an analytic action of a $p$-adic Lie group to which Lie theory may be applied. This is how Theorems A and B are proven; our strategy applies also to actions of other discrete groups, such as the mapping class group of a closed surface of genus $g$, or the group of outer automorphisms of a free group (see $\S 6$ and $\S[1]$ ). Let us state a sample result. 
Given a group $\Gamma$, let $\mathrm{ma}(\Gamma)$ be the smallest dimension of a complex irreducible variety on which some finite-index subgroup of $\Gamma$ acts faithfully by automorphisms. Let $\operatorname{Mod}(g)$ be the mapping class group of a closed orientable surface of genus $g$. It is known that $\operatorname{ma}(\operatorname{Mod}(g)) \leqslant 6 g-6$ for all $g \geqslant 2(\operatorname{see} \S 6.1)$, and that $\operatorname{ma}(\operatorname{Mod}(1))=1$ (because a finiteindex subgroup of $\mathrm{GL}_{2}(\mathbf{Z})$ embeds into $\mathrm{PGL}_{2}(\mathbf{C})$ ).

TheOREM D. If $\operatorname{Mod}(g)$ acts faithfully on a complex variety $X$ by automorphisms, then $\operatorname{dim}(X) \geqslant 2 g-1$. Thus, $2 g-1 \leqslant \operatorname{ma}(\operatorname{Mod}(g)) \leqslant 6 g-6$ for every $g \geqslant 2$.

\subsection{Margulis super-rigidity and Zimmer program}

Let $\Gamma$ be a lattice in a simple real Lie group $S$, with $\operatorname{rank}_{\mathbf{R}}(S) \geqslant 2$. According to the Margulis super-rigidity theorem, unbounded linear representations of the discrete group $\Gamma$ "come from" linear algebraic representations of the group $S$ itself. As a byproduct, the smallest dimension of a faithful linear representation of $\Gamma$ coincides with the smallest dimension of a faithful linear representation of S (see [51]).

The Zimmer program asks for an extension of this type of rigidity results to nonlinear actions of $\Gamma$, for instance to actions of $\Gamma$ by diffeomorphisms on compact manifolds (see [67], [68], and the recent survey [32]). Theorems A and B are instances of Zimmer program in the context of algebraic geometry.

When $\Gamma=\mathrm{SL}_{n}(\mathbf{Z})$ or $\Gamma=\mathrm{Sp}_{2 n}(\mathbf{Z})$, Bass, Milnor, and Serre obtained a super-rigidity theorem from their solution of the congruence subgroup problem (see $[2, \S 16]$ and [62]). Our proofs of Theorems A and B may be considered as extensions of their argument to the context of non-linear actions by algebraic transformations.

\subsection{Notation}

To specify the field (or ring) of definition $K$ of an algebraic variety (or scheme) $X$, we use the notation $X_{K}$. If $K^{\prime}$ is an extension of $K, X\left(K^{\prime}\right)$ is the set of $K^{\prime}$-points of $X$. The group of automorphisms (resp. birational transformations) of $X$ which are defined over $K^{\prime}$ is denoted Aut $\left(X_{K^{\prime}}\right)\left(\operatorname{resp}\right.$. $\left.\operatorname{Bir}\left(X_{K^{\prime}}\right)\right)$.

\subsection{Acknowledgement}

Thanks to Yves de Cornulier, Julie Déserti, Philippe Gille, Sébastien Gouezel, Vincent Guirardel, and Peter Sarnak for interesting discussions related to this article. We thank the referees for numerous insightful remarks, and in particular for their suggestions regarding $\S 2.4, \S 6$ and $\S 7$. This work was supported by the ANR project BirPol and 
the foundation Del Duca from the French Academy of Sciences, and the Institute for Advanced Study, Princeton.

\section{Tate analytic diffeomorphisms of the $p$-adic polydisk}

In this section, we introduce the group of Tate analytic diffeomorphisms of the unit polydisk $\mathcal{U}=\mathbf{Z}_{p}^{d}$, describe its topology, and study its finite-dimensional subgroups. The main result of this section is Theorem 2.11.

\subsection{Tate analytic diffeomorphisms}

\subsubsection{The Tate algebra (see $[59, \S 6])$}

Let $p$ be a prime number. Let $K$ be a field of characteristic zero which is complete with respect to an absolute value $|\cdot|$ satisfying $|p|=1 / p$; such an absolute value is automatically ultrametric (see [44, Examples 2 and 3 in $\S I .2]$ ). Good examples to keep in mind are the fields of $p$-adic numbers $\mathbf{Q}_{p}$ and its finite extensions. Let $R$ be the valuation ring of $K$, i.e. the subset of $K$ defined by $R=\{x \in K:|x| \leqslant 1\}$; in the vector space $K^{d}$, the unit polydisk is the subset $R^{d}$.

Fix a positive integer $d$, and consider the ring $R[\mathbf{x}]=R\left[\mathbf{x}_{1}, \ldots, \mathbf{x}_{d}\right]$ of polynomial functions in $d$ variables with coefficients in $R$. For $f$ in $R[\mathbf{x}]$, define the norm $\|f\|$ to be the supremum of the absolute values of the coefficients of $f$ :

$$
\|f\|=\sup _{I}\left|a_{I}\right|
$$

where $f=\sum_{I=\left(i_{1}, \ldots, i_{d}\right)} a_{I} \mathbf{x}^{I}$. By definition, the Tate algebra $R\langle\mathbf{x}\rangle$ is the completion of $R[\mathbf{x}]$ with respect to the norm $\|\cdot\|$. The Tate algebra coincides with the set of formal power series $f=\sum_{I} a_{I} \mathbf{x}^{I}, I \in \mathbf{Z}_{+}^{d}$, converging (absolutely) on the closed unit polydisk $R^{d}$. Moreover, the absolute convergence is equivalent to $\left|a_{I}\right| \rightarrow 0$ as $\|I\| \rightarrow \infty$.

For $f$ and $g$ in $R\langle\mathbf{x}\rangle$ and $c$ in $\mathbf{R}_{+}$, the notation $f \in p^{c} R\langle\mathbf{x}\rangle$ means $\|f\| \leqslant|p|^{c}$ and

$$
f \equiv g\left(\bmod p^{c}\right)
$$

means $\|f-g\| \leqslant|p|^{c}$; we then extend such notation componentwise to $(R\langle\mathbf{x}\rangle)^{m}$ for all $m \geqslant 1$. For instance, with $d=2$, the polynomial mapping $f(\mathbf{x})=\left(\mathbf{x}_{1}+p, \mathbf{x}_{2}+p \mathbf{x}_{1} \mathbf{x}_{2}\right)$ satisfies $f \equiv \mathrm{id}(\bmod p)$, where $\mathrm{id}(\mathbf{x})=\mathbf{x}$ is the identity. 


\subsubsection{Tate diffeomorphisms}

Denote by $\mathcal{U}$ the unit polydisk of dimension $d$, that is $\mathcal{U}=R^{d}$. For $x$ and $y$ in $\mathcal{U}$, the distance $\operatorname{dist}(x, y)$ is defined by $\operatorname{dist}(x, y)=\max _{i}\left|x_{i}-y_{i}\right|$, where the $x_{i}$ 's and $y_{i}$ 's are the coordinates of $x$ and $y$ in $R^{d}$. The non-archimedean triangle inequality implies that $|h(y)| \leqslant 1$ for every $h$ in $R\langle\mathbf{x}\rangle$ and $y \in \mathcal{U}$. Consequently, every element $g$ in $R\langle\mathbf{x}\rangle^{d}$ determines a Tate analytic map $g: \mathcal{U} \rightarrow \mathcal{U}$.

If $g=\left(g_{1}, \ldots, g_{d}\right)$ is an element of $R\langle\mathbf{x}\rangle^{d}$, the norm $\|g\|$ is defined as the maximum of the norms $\left\|g_{i}\right\|$ (see equation (2.1)); one has

$$
\|g\| \leqslant 1 \quad \text { and } \quad \operatorname{dist}(g(x), g(y)) \leqslant\|g\| \operatorname{dist}(x, y),
$$

so that $g$ is 1-Lipschitz.

For indeterminates $\mathbf{x}=\left(\mathbf{x}_{1}, \ldots, \mathbf{x}_{d}\right)$ and $\mathbf{y}=\left(\mathbf{y}_{1}, \ldots, \mathbf{y}_{m}\right)$, the composition

$$
R\langle\mathbf{y}\rangle \times R\langle\mathbf{x}\rangle^{m} \longrightarrow R\langle\mathbf{x}\rangle
$$

is well defined, and hence coordinatewise we obtain

$$
R\langle\mathbf{y}\rangle^{n} \times R\langle\mathbf{x}\rangle^{m} \longrightarrow R\langle\mathbf{x}\rangle^{n}
$$

In particular, with $m=n=d$, we get a semigroup $R\langle\mathbf{x}\rangle^{d}$. The group of (Tate) analytic diffeomorphisms of $\mathcal{U}$ is the group of invertible elements in this semigroup; we denote it by $\operatorname{Diff}^{\mathrm{an}}(\mathcal{U})$. Elements of $\operatorname{Diff}^{\mathrm{an}}(\mathcal{U})$ are transformations $f: \mathcal{U} \rightarrow \mathcal{U}$ given by

$$
f(\mathbf{x})=\left(f_{1}, \ldots, f_{d}\right)(\mathbf{x}),
$$

where each $f_{i}$ is in $R\langle\mathbf{x}\rangle$ and $f$ has an inverse $f^{-1}: \mathcal{U} \rightarrow \mathcal{U}$ that is also defined by power series in the Tate algebra. The distance between two Tate analytic diffeomorphisms $f$ and $g$ is defined as $\|f-g\|$; by the following lemma, this endows $\operatorname{Diff}^{\text {an }}(\mathcal{U})$ with the structure of a topological group.

Lemma 2.1. Let $f, g$, and $h$ be elements of $R\langle\mathbf{x}\rangle^{d}$. Then,

(1) $\|g \circ f\| \leqslant\|g\|$;

(2) if $f$ is an element of $\operatorname{Diff}^{a n}(\mathcal{U})$, then $\|g \circ f\|=\|g\|$;

(3) $\|g \circ(\mathrm{id}+h)-g\| \leqslant\|h\|$;

(4) $\left\|f^{-1}-\mathrm{id}\right\|=\|f-\mathrm{id}\|$ if $f$ is a Tate analytic diffeomorphism.

Proof. Let $s \in R$ and $c>0$ satisfy $|p|^{c}=|s|=\|g\|$. Then $(1 / s) g$ is an element of $R\langle\mathbf{x}\rangle^{d}$. It follows that $(1 / s) g \circ f$ is an element of $R\langle\mathbf{x}\rangle^{d}$ too, and that $\|g \circ f\| \leqslant|p|^{c}$. This proves 
assertion (1). The second assertion follows because $g=(g \circ f) \circ f^{-1}$. To prove assertion (3), write $h=\left(h_{1}, h_{2}, \ldots, h_{d}\right)$, where each $h_{i}$ satisfies $\left\|h_{i}\right\| \leqslant\|h\|$. Then $g \circ(\mathrm{id}+h)$ takes the form

$$
g \circ(\mathrm{id}+h)=g+A_{1}(h)+\sum_{i \geqslant 2} A_{i}(h)
$$

where each $A_{i}$ is a homogeneous polynomial in $\left(\mathbf{x}_{1}, \ldots, \mathbf{x}_{d}\right)$ of degree $i$ with coefficients in $R$. Assertion (3) follows. For assertion (4), assume that $f$ is an analytic diffeomorphism and apply assertion (2): $\left\|f^{-1}-\mathrm{id}\right\|=\|\mathrm{id}-f\|$.

This lemma easily implies the following proposition (see [18] for details).

Proposition 2.2. For every real number $c>0$, the subgroup of all elements $f \in$ $\operatorname{Diff}^{\mathrm{an}}(\mathcal{U})$ with $f \equiv \mathrm{id}\left(\bmod p^{c}\right)$ is a normal subgroup of $\operatorname{Diff}^{\mathrm{an}}(\mathcal{U})$.

Lemma 2.3. Let $f$ be an element of $\operatorname{Diff}^{\mathrm{an}}(\mathcal{U})$. If $f(\mathbf{x}) \equiv \mathrm{id}\left(\bmod p^{c}\right)$, with $c \geqslant 1$, and $p^{N}$ divides $l$, then the $l$-th iterate of $f$ satisfies $f^{l}(\mathbf{x}) \equiv \mathrm{id}\left(\bmod p^{c+N}\right)$. In particular, if $f \equiv \mathrm{id}(\bmod p)$, then $f^{p^{\ell}} \equiv \mathrm{id}\left(\bmod p^{\ell}\right)$.

Proof. Write $f(\mathbf{x})=\mathbf{x}+\operatorname{sr}(\mathbf{x})$, where $r$ is in $R\langle\mathbf{x}\rangle^{d}$ and $s \in R$ satisfies $|s| \leqslant|p|^{c}$. Then,

$$
f \circ f(\mathbf{x})=\mathbf{x}+s r(\mathbf{x})+s r(\mathbf{x}+s r(\mathbf{x}))=\mathbf{x}+2 s r(\mathbf{x})+s^{2} u_{2}(\mathbf{x})
$$

for some $u_{2} \in R\langle\mathbf{x}\rangle^{d}$. After $k$ iterations, one gets

$$
f^{k}(\mathbf{x})=\mathbf{x}+k s r(\mathbf{x})+s^{2} u_{k}(\mathbf{x})
$$

with $u_{k} \in R\langle\mathbf{x}\rangle^{d}$. Taking $k=p$, we obtain

$$
f^{p}(\mathbf{x})=\mathbf{x}+p s r(\mathbf{x})+s^{2} u_{p}(\mathbf{x}) \equiv \mathbf{x}\left(\bmod p^{c+1}\right)
$$

because $c \geqslant 1$. Then, $f^{p^{2}}(\mathbf{x}) \equiv \mathbf{x}\left(\bmod p^{c+2}\right)$ and $f^{p^{N}}(\mathbf{x}) \equiv \mathbf{x}\left(\bmod p^{c+N}\right)$.

\subsection{From cyclic groups to $p$-adic flows}

\subsubsection{From cyclic groups to $R$-flows}

The following theorem is due to Bell and to Poonen (see [55], as well as [6, Lemma 4.2] and [7, Theorem 3.3]).

THEOREM 2.4. Let $f$ be an element of $R\langle\mathbf{x}\rangle^{d}$ with $f \equiv \mathrm{id}\left(\bmod p^{c}\right)$ for some real number $c>1 /(p-1)$. Then, $f$ is a Tate diffeomorphism of $\mathcal{U}=R^{d}$ and there exists a unique Tate analytic map $\Phi: \mathcal{U} \times R \rightarrow \mathcal{U}$ such that

(1) $\Phi(\mathbf{x}, n)=f^{n}(\mathbf{x})$ for all $n \in \mathbf{Z}$; 
(2) $\Phi(\mathbf{x}, t+s)=\Phi(\Phi(\mathbf{x}, s), t)$ for all $t, s \in R$;

(3) $\Phi: t \in R \mapsto \Phi(\cdot, t)$ is a continuous homomorphism from the abelian group $(R,+)$ to the group of Tate diffeomorphisms $\operatorname{Diff}^{\mathrm{an}}(\mathcal{U})$;

(4) $\Phi(\mathbf{x}, t) \equiv \mathbf{x}\left(\bmod p^{c-1 /(p-1)}\right)$ for all $t \in R$.

We shall refer to this theorem as the "Bell-Poonen theorem", or "Bell-Poonen extension theorem". An analytic map $\Phi: \mathcal{U} \times R \rightarrow \mathcal{U}$ which defines an action of the group $(R,+)$ will be called an $R$-flow, or simply a flow. See below, in $\S 2.2 .2$, how it is viewed as the flow of an analytic vector field. A flow $\Phi$ will be considered either as an analytic action $\Phi: \mathcal{U} \times R \rightarrow \mathcal{U}$ of the abelian group $(R,+)$, or as a morphism

$$
\Phi: t \in R \longmapsto \Phi_{t}=\Phi(\cdot, t) \in \operatorname{Diff}^{\mathrm{an}}(\mathcal{U}) ;
$$

we use the same vocabulary (and the same letter $\Phi$ ) for the two maps. The Bell-Poonen theorem implies that every element $f$ of $R\langle\mathbf{x}\rangle^{d}$ with $f \equiv \mathrm{id}\left(\bmod p^{2}\right)$ is included in an analytic $R$-flow.

Corollary 2.5. Let $f$ be an element of $R\langle\mathbf{x}\rangle^{d}$ with $f \equiv \mathrm{id}\left(\bmod p^{c}\right)$ for some real number $c>1 /(p-1)$. Then, $f$ is a Tate diffeomorphism of $\mathcal{U}=R^{d}$ and, if $f$ has finite order in $\operatorname{Diff}^{a}(\mathcal{U})$, then $f=\mathrm{id}$.

To prove it, assume that $f$ has order $k \geqslant 1$ and apply the Bell-Poonen theorem. For every $x \in \mathcal{U}$, the analytic curve $t \mapsto \Phi(x, t)-x$ vanishes on the infinite set $\mathbf{Z} k$; hence, it vanishes identically, $f(x)=\Phi(x, 1)=x$ for all $x \in \mathcal{U}$, and $f=\mathrm{id}$.

Remark 2.6. Theorem 2.4 is not stated as such in [55]. Poonen constructs a Tate analytic map $\Phi: \mathcal{U} \times R \rightarrow \mathcal{U}$ which satisfies property (1) for $n \geqslant 0$; his proof implies also Properties (3) and (4). We now deduce property (1) for $n \in \mathbf{Z}$. We already know that the relation $\Phi(\mathbf{x}, n+1)=f \circ \Phi(\mathbf{x}, n)$ holds for every integer $n \geqslant 0$. Thus, for every $x$ in $\mathcal{U}$, the two Tate analytic functions $t \mapsto \Phi(x, t+1)$ and $t \mapsto f \circ \Phi(x, t)$ coincide on $\mathbf{Z}_{+}$, and hence on $R$, by the isolated zero principle. This implies $\Phi(\mathbf{x}, t+1)=f \circ \Phi(\mathbf{x}, t)$ in $R\langle\mathbf{x}, t\rangle^{d}$. Take $t=-1$ to deduce that $f$ is an analytic diffeomorphism of $\mathcal{U}$ and $f^{-1}=\Phi(\cdot,-1)$. Then, by induction, one gets $\Phi(\mathbf{x}, n)=f^{n}(\mathbf{x})$ for all $n \in \mathbf{Z}$. Property (2) follows from (1) for $s$ and $t$ in $\mathbf{Z}$, and then for all values of $s$ and $t$ in $R$ by the isolated zero principle.

\subsubsection{Flows and Tate analytic vector fields}

Consider the Lie algebra $\Theta(\mathcal{U})$ of vector fields $\mathbf{X}=\sum_{i=1}^{d} u_{i}(\mathbf{x}) \partial_{i}$, where each $u_{i}$ is an element of the Tate algebra $R\langle\mathbf{x}\rangle$. The Lie bracket with a vector field $\mathbf{Y}=\sum_{i} v_{i}(\mathbf{x}) \partial_{i}$ is given by

$$
[\mathbf{X}, \mathbf{Y}]=\sum_{j=1}^{d} w_{j}(\mathbf{x}) \partial_{j}, \quad \text { with } w_{j}=\sum_{i=1}^{d}\left(u_{i} \frac{\partial v_{j}}{\partial x_{i}}-v_{i} \frac{\partial u_{j}}{\partial x_{i}}\right)
$$


Lemma 2.7. Let $\Phi: \mathcal{U} \times R \rightarrow \mathcal{U}$ be an element of $R\langle\mathbf{x}, t\rangle^{d}$ that defines an analytic flow. Then, $\mathbf{X}=\left.(\partial \Phi / \partial t)\right|_{t=0}$ is an analytic vector field. It is preserved by $\Phi_{t}$ : for all $t \in R,\left(\Phi_{t}\right)_{*} \mathbf{X}=\mathbf{X}$. Moreover, $\mathbf{X}\left(x_{0}\right)=\mathbf{0}$ if and only if $\Phi_{t}\left(x_{0}\right)=x_{0}$ for all $t \in R$.

The analyticity and $\Phi_{t}$-invariance are easily obtained. Let us show that $\mathbf{X}\left(x_{0}\right)=0$ if and only if $x_{0}$ is a fixed point of $\Phi_{t}$ for all $t$. Indeed, if $\mathbf{X}$ vanishes at $x_{0}$, then $\mathbf{X}$ vanishes along the curve $\Phi\left(x_{0}, t\right), t \in R$, because $\mathbf{X}$ is $\Phi_{t}$-invariant. Thus, $\partial_{t} \Phi\left(x_{0}, t\right)=0$ for all $t$, and the result follows.

Corollary 2.8. If $f$ is an element of $\operatorname{Diff}^{\mathrm{an}}(\mathcal{U})$ with $f \equiv \mathrm{id}\left(\bmod p^{c}\right)$ for some $c>1 /(p-1)$, then $f$ is given by the flow $\Phi_{f}$, at time $t=1$, of a unique analytic vector field $\mathbf{X}_{f}$. The zeros of $\mathbf{X}_{f}$ are the fixed points of $f$.

Two such diffeomorphisms $f$ and $g$ commute if and only if $\left[\mathbf{X}_{f}, \mathbf{X}_{g}\right]=\mathbf{0}$.

Proof. The first assertion follows directly from Lemma 2.7 and the Bell-Poonen theorem (Theorem 2.4). Let us prove the second assertion. If $\mathbf{X}_{f}$ commute with $\mathbf{X}_{g}$, then $\Phi_{f}$ and $\Phi_{g}$ commute too, meaning that $\Phi_{f}\left(\Phi_{g}(x, t), s\right)=\Phi_{g}\left(\Phi_{f}(x, s), t\right)$ for every pair $(s, t) \in R \times R$. Taking $(s, t)=(1,1)$, we obtain $f \circ g=g \circ f$. If $f$ and $g$ commute, then $\Phi_{f}\left(\Phi_{g}(x, n), m\right)=f^{m} \circ g^{n}=\Phi_{g}\left(\Phi_{f}(x, m), n\right)$ for every pair $(m, n)$ of integers, and the principle of isolated zeros implies that the flows $\Phi_{f}$ and $\Phi_{g}$ commute; hence, $\left[\mathbf{X}_{f}, \mathbf{X}_{g}\right]=\mathbf{0}$.

\subsection{A pro- $p$ structure}

Recall that a pro-p group is a topological group $G$ which is a compact Hausdorff space, with a basis of neighborhoods of the neutral element $1_{G}$ generated by subgroups having a (finite) power of $p$ as index. In such a group, the index of every open normal subgroup is a power of $p$. We refer to [28] for a good introduction to pro- $p$ groups.

In this subsection, we assume that $K$ is a finite extension of $\mathbf{Q}_{p}$. The residue field, i.e. the quotient of $R$ by its maximal ideal $\mathbf{m}_{K}=\{x \in K:|x|<1\}$, is a finite field of characteristic $p$. It has $q$ elements, with $q$ being a power of $p$, and the number of elements of the ring $R / \mathbf{m}_{K}^{k}$ is a power of $p$ for every $k$. We also fix an element $\pi$ that generates the ideal $\mathbf{m}_{K}$.

\subsubsection{Action modulo $\mathrm{m}_{K}^{k}$}

Recall that $\mathcal{U}$ denotes the polydisk $R^{d}$. Let $f$ be an element of $\operatorname{Diff}^{\text {an }}(\mathcal{U})$. Its reduction modulo $\mathbf{m}_{K}^{k}$ is a polynomial transformation with coefficients $\bar{a}_{I}$ in the finite ring $R / \mathbf{m}_{K}^{k}$; it determines a permutation of the finite set $\left(R / \mathbf{m}_{K}^{k}\right)^{d}$. Thus, for each $k \geqslant 1$, reduction 
modulo $\mathbf{m}_{K}^{k}$ provides a homomorphism $\theta_{k}: \operatorname{Diff}^{\mathrm{an}}(\mathcal{U}) \rightarrow \operatorname{Perm}\left(\left(R / \mathbf{m}_{K}^{k}\right)^{d}\right)$ into the group of permutations of the finite set $\left(R / \mathbf{m}_{K}^{k}\right)^{d}$.

Another way to look at the same action is as follows. Each element of $\operatorname{Diff}^{\text {an }}(\mathcal{U})$ acts isometrically on $\mathcal{U}$ with respect to the distance $\operatorname{dist}(x, y)$ (see $\S 2.1 .2$ ); in particular, for every radius $r$, $\operatorname{Diff}^{\text {an }}(\mathcal{U})$ acts by permutations on the set of balls of $\mathcal{U}$ of radius $r$. Since the set of balls of radius $|\pi|^{-k}$ is in bijection with the set $\left(R / \mathbf{m}_{K}^{k}\right)^{d}$, the action of $\operatorname{Diff}^{\text {an }}(\mathcal{U})$ on this set of balls may be identified with its action on $\left(R / \mathbf{m}_{K}^{k}\right)^{d}$ after reduction modulo $\mathbf{m}_{K}^{k}$.

As a consequence, we have that an element $f$ of $\operatorname{Diff}^{\mathrm{an}}(\mathcal{U})$ is the identity if and only if $\operatorname{dist}(f(x), x) \leqslant|\pi|^{k}$ for all $x$ and all $k$, and this is true if and only if its image in the group of permutations of $\left(R / \mathbf{m}_{K}^{k}\right)^{d}$ is trivial for all $k$.

\subsubsection{A pro- $p$ completion}

Given a positive integer $r$, define $\operatorname{Diff}^{\mathrm{an}}(\mathcal{U})_{r}$ as the subgroup of $\operatorname{Diff}^{\mathrm{an}}(\mathcal{U})$ whose elements are equal to the identity modulo $p^{r}$. For $r=1$, we set

$$
D=\operatorname{Diff}^{\mathrm{an}}(\mathcal{U})_{1}=\left\{f \in \operatorname{Diff}^{\mathrm{an}}(\mathcal{U}): f \equiv \mathrm{id}(\bmod p)\right\}
$$

By definition, $f$ is an element of $D$ if it can be written $f=\mathrm{id}+p h$, where $h$ is in $R\langle\mathbf{x}\rangle^{d}$. Thus, $D$ acts trivially on $(R / p R)^{d}$ (here, $p R=\mathbf{m}_{K}^{\ell}$ with $\left|\pi^{\ell}\right|=1 / p$ for some $\ell$ ).

Now we show that the image $\theta_{\ell m}(D)$ in $\operatorname{Perm}\left(\left(R / p^{m} R\right)^{d}\right)$ is a finite $p$-group. Indeed, by Lemma 2.3 , for any $f \in D$, we have $f^{p^{m-1}} \equiv \mathrm{id}\left(\bmod p^{m}\right)$. Thus, $f^{p^{m-1}}$ acts trivially on $\left(R / p^{m} R\right)^{d}$. It follows that the order of every element in $\theta_{\ell m}(D)$ is a power of $p$. Since $\theta_{\ell m}(D)$ is a finite group, Sylow's theorem implies that $\theta_{\ell m}(D)$ is a $p$-group.

We endow the finite groups $\operatorname{Perm}\left(\left(R / p^{m} R\right)^{d}\right)$ with the discrete topology, and we denote by $\widehat{D}$ the inverse limit of the $p$-groups $\theta_{\ell m}(D) \subset \operatorname{Perm}\left(\left(R / p^{m} R\right)^{d}\right) ; \widehat{D}$ is a pro$p$ group: It is the closure of the image of $D$ in $\prod_{m} \operatorname{Perm}\left(\left(R / p^{m} R\right)^{d}\right)$ by the diagonal embedding $\left(\theta_{\ell m}\right)_{m \geqslant 1}$. We denote by $\mathcal{T}$ the topology of $\widehat{D}$ (resp. the induced topology on $D)$; the kernels of the homomorphisms $\theta_{\ell m}$ form a basis of neighborhoods of the identity for this topology.

Since the action on $\left(R / p^{m} R\right)^{d}$ is the action on the set of balls of radius $p^{-m}$ in $\mathcal{U}$, the Tate topology is finer than the topology $\mathcal{T}$ : The identity map $f \mapsto f$ is a continuous homomorphism with respect to the Tate topology on the source, and the topology $\mathcal{T}$ on the target; we shall denote this continuous injective homomorphism by

$$
D \ni f \longmapsto \hat{f} \in \widehat{D}
$$


Remark 2.9. Fix a prime $p$ and consider the field $K=\mathbf{Q}_{p}$, with valuation $\operatorname{ring} R=\mathbf{Z}_{p}$. Assume that the dimension $d$ is 2 . The sequence of polynomial automorphisms of the affine plane defined by $h_{n}(x, y)=\left(x, y+p\left(x+x^{2}+x^{3}+\ldots+x^{n}\right)\right)$ determines a sequence of elements of $D$. No subsequence of $\left\{h_{n}\right\}_{n}$ converges in the Tate topology, but in the compact group $\widehat{D}$ one can extract a converging subsequence. A better example is provided by the sequence $g_{n}(x, y)=\left(x, y+s_{n}(x)\right)$ with $s_{n}(x)=x^{n}\left(x^{p^{n}-p^{n-1}}-1\right)$. This sequence converges towards the identity in $\widehat{D}$, because $s_{n}$ vanishes on $\mathbf{Z} / p^{n} \mathbf{Z}$, but does not converge in $D$ for the Tate topology.

\subsection{Extension theorem}

\subsubsection{Analytic groups (see [47, §IV], or [28], [60] and [10, Chapter III])}

Let $G$ be a topological group. We say that $G$ is a $p$-adic analytic group if there is a structure of $p$-adic analytic manifold on $G$ which is compatible with the topology of $G$ and the group structure. The group law $G \times G \ni(x, y) \mapsto x y^{-1}$ is $p$-adic analytic (see [28, Chapter 8]). If such a structure exists, it is unique (see [28, Chapter 9]). The dimension $\operatorname{dim}(G)$ is the dimension of $G$ as a $p$-adic manifold.

If $G$ is a compact, $p$-adic analytic group, then $G$ contains a finite-index, open, normal subgroup $G_{0}$ which is a (uniform) pro- $p$ group. Moreover, $G_{0}$ embeds continuously in $\mathrm{GL}_{d}\left(\mathbf{Z}_{p}\right)$ for some $d$ (see [28, Chapters 7 and 8$]$ ).

Let $g$ be an element of the pro- $p$ group $G$. The homomorphism $\varphi: m \in \mathbf{Z} \mapsto g^{m} \in G$ extends automatically to the pro- $p$ completion of $\mathbf{Z}$, i.e. to a continuous homomorphism of pro- $p$ groups

$$
\bar{\varphi}: \mathbf{Z}_{p} \longrightarrow G .
$$

For simplicity, we denote $\bar{\varphi}(t)$ by $g^{t}$ for $t$ in $\mathbf{Z}_{p}$ (see [28, Proposition 1.28], for embeddings of $\mathbf{Z}_{p}$ into pro- $p$ groups).

Lemma 2.10. Let $G$ be a p-adic analytic pro-p group of dimension $s=\operatorname{dim}(G)$. Let $\Gamma$ be a dense subgroup of $G$. There exist an integer $r \geqslant s$ and elements $\gamma_{1}, \ldots, \gamma_{r}$ in $\Gamma$ such that the map $\pi: \mathbf{Z}_{p}^{r} \rightarrow G, \pi\left(t_{1}, \ldots, t_{r}\right)=\left(\gamma_{1}\right)^{t_{1}} \ldots\left(\gamma_{r}\right)^{t_{r}}$, satisfies the following properties:

(1) $\pi$ is a surjective, $p$-adic analytic map;

(2) the restriction of $\pi$ to $\left\{\left(t_{i}\right)_{i}: t_{j}=0\right.$ if $\left.j>s\right\}$ is a local diffeomorphism onto its image;

(3) as $l$ runs over the set of positive integers, the sets $\pi\left(\left(p^{l} \mathbf{Z}_{p}\right)^{r}\right)$ form a basis of neighborhoods of the neutral element in $G$.

Proof. Let $\mathfrak{g}$ be the Lie algebra of $G$; as a finite-dimensional $\mathbf{Q}_{p}$-vector space, $\mathfrak{g}$ coincides with the tangent space of $G$ at the neutral element $1_{G}$. There are finite- 
index open subgroups $H$ of $G$ for which the exponential map defines a $p$-adic analytic diffeomorphism from a neighborhood of the origin in $\mathfrak{g}\left(\mathbf{Z}_{p}\right)$ onto the group $H$ itself (see the notion of standard subgroups in [10], [28]). Let $H$ be such a subgroup.

Since $\Gamma$ is dense in $G$, its intersection with $H$ is dense in $H$. Each $\alpha_{i} \in \Gamma \cap H$ corresponds to a tangent vector $\nu_{i} \in \mathfrak{g}$ such that $\exp \left(t \nu_{i}\right)=\alpha_{i}^{t}$ for $t \in \mathbf{Z}_{p}$. Since $\Gamma$ is dense in $H$, the subspace of $\mathfrak{g}$ generated by all the $\nu_{i}$ is equal to $\mathfrak{g}$. Thus, one can find elements $\alpha_{1}, \ldots, \alpha_{s}$ of $\Gamma$, with $s=\operatorname{dim}(G)$, such that the $\nu_{i}$ generate $\mathfrak{g}$. Then, the map $\pi: \mathbf{Z}_{p}^{d} \rightarrow H$ defined by

$$
\pi\left(t_{1}, \ldots, t_{s}\right)=\exp \left(t_{1} \nu_{1}\right) \ldots \exp \left(t_{s} \nu_{s}\right)=\alpha_{1}^{t_{1}} \ldots \alpha_{s}^{t_{s}}
$$

is analytic and, by the $p$-adic inverse function theorem, it determines a local analytic diffeomorphism from a neighborhood of zero in $\mathfrak{g}$ to a neighborhood $V$ of $1_{G}$. The group $G$ can then be covered by a finite number of translates $h_{j} V, j=1, \ldots, s^{\prime}$. Since $\Gamma$ is dense, one can find elements $\beta_{j}$ in $\Gamma$ with $\beta_{j}^{-1} h_{j} \in V$. The lemma follows if one sets $r=s+s^{\prime}$, $\gamma_{i}=\alpha_{i}$ for $1 \leqslant i \leqslant s$, and $\gamma_{i}=\beta_{i-s}$ for $s+1 \leqslant i \leqslant r$.

\subsubsection{Actions by Tate analytic diffeomorphisms.}

Let $G$ be a compact $p$-adic analytic group. Let $\Gamma$ be a finitely generated subgroup of $G$. We say that $G$ is a virtual pro-p completion of $\Gamma$ if there exists a finite-index subgroup $\Gamma_{0}$ of $\Gamma$ such that (1) the closure of $\Gamma_{0}$ in $G$ is an open pro- $p$ subgroup $G_{0}$ of $G$, and (2) $G_{0}$ coincides with the pro- $p$ completion of $\Gamma_{0}$. Note that, by compactness of $G$, the group $G_{0}$ has finite index in $G$. A good example to keep in mind is $\Gamma=\mathrm{SL}_{n}(\mathbf{Z})$ in $G=\mathrm{SL}_{n}\left(\mathbf{Z}_{p}\right)$ (see $§ 4.2 .3$ below).

We now study homomorphisms from $\Gamma$ to the group $\operatorname{Diff}^{a n}(\mathcal{U})$. Thus, in this paragraph, the same prime number $p$ plays two roles, since it appears in the definition of the pro- $p$ structure of $G$, and of the Tate topology on $\operatorname{Diff}^{\text {an }}(\mathcal{U})$.

TheOREM 2.11. Let $p$ be an odd prime, and let $G$ be a compact, $p$-adic analytic group. Let $\Gamma$ be a finitely generated subgroup of $G$. Assume that $G$ is a virtual pro- $p$ completion of $\Gamma$.

Let $\Phi: \Gamma \rightarrow \operatorname{Diff}^{\text {an }}(\mathcal{U})_{1}$ be a homomorphism into the group of Tate analytic diffeomorphisms of $\mathcal{U}$ which are equal to the identity modulo $p$. Then, there exists a finite-index subgroup $\Gamma_{0}$ of $\Gamma$ for which $\left.\Phi\right|_{\Gamma_{0}}$ extends to the closure $G_{0}=\bar{\Gamma}_{0} \subset G$ as a continuous homomorphism

$$
\bar{\Phi}: G_{0} \longrightarrow \operatorname{Diff}^{\text {an }}(\mathcal{U})_{1}
$$

such that the action $G_{0} \times \mathcal{U} \rightarrow \mathcal{U}$ given by $(g, x) \mapsto \bar{\Phi}(g)(x)$ is analytic. 
Denote $\operatorname{Diff}^{\text {an }}(\mathcal{U})_{1}$ by $D$, as in equation (2.4). Recall from $\S 2.3 .2$ that $D$ embeds continuously into the pro- $p$ group $\widehat{D}$. Let $\Gamma_{0}$ be a finite-index subgroup of $\Gamma$ whose closure $G_{0}$ in $G$ is the pro- $p$ completion of $\Gamma_{0}$. We obtain:

(1) The homomorphism $\Gamma_{0} \rightarrow \widehat{D}$ extends uniquely into a continuous homomorphism $\hat{\Phi}$ from $G_{0}=\bar{\Gamma}_{0}$ to $\widehat{D}$.

Then, the following property is automatically satisfied:

(2) Let $f$ be an element of $D$. By the Bell-Poonen extension theorem (Theorem 2.4), the homomorphism $t \in \mathbf{Z} \mapsto f^{t}$ extends to a continuous morphism $\mathbf{Z}_{p} \rightarrow D$ via a Tate analytic flow. If $\hat{f}$ denotes the image of $f$ in $\widehat{D}$, then $n \mapsto(\hat{f})^{n}$ is a homomorphism from $\mathbf{Z}$ to the pro- $p$ group $\widehat{D}$; as such, it extends canonically to the pro- $p$ completion $\mathbf{Z}_{p}$, giving rise to a homomorphism $t \in \mathbf{Z}_{p} \mapsto(\hat{f})^{t} \in \widehat{D}$. These two extensions are compatible: $\widehat{\left(f^{t}\right)}=(\hat{f})^{t}$ for all $t$ in $\mathbf{Z}_{p}$.

Thus, given any 1-parameter subgroup $\mathbf{Z}$ of $\Gamma$, we already know how to extend $\Phi: \mathbf{Z} \subset$ $\Gamma \rightarrow D$ into $\bar{\Phi}: \mathbf{Z}_{p} \subset G_{0} \rightarrow D$ in a way that is compatible with the extension $\hat{\Phi}: G_{0} \rightarrow \widehat{D}$.

For simplicity, we now denote $\Gamma_{0}$ by $\Gamma$ and $G_{0}$ and $G$.

Lemma 2.12. Let $\left(\alpha_{n}\right)_{n}$ be a sequence of elements of $\Gamma$ that converges towards $1_{G}$ in $G$. Then $\left(\Phi\left(\alpha_{n}\right)\right)_{n}$ converges towards the identity in $\operatorname{Diff}^{\text {an }}(\mathcal{U})$.

Proof. Write $\alpha_{n}=\pi\left(t_{1}(n), \ldots, t_{r}(n)\right)=\left(\gamma_{1}\right)^{t_{1}(n)} \ldots\left(\gamma_{r}\right)^{t_{r}(n)}$, where $\pi$ and the $\gamma_{i}$ are given by Lemma 2.10 and the $t_{i}(n)$ are in $\mathbf{Z}_{p}$. Since $\alpha_{n}$ converges towards $1_{G}$, we may assume, by Lemma 2.10, that each $\left(t_{i}(n)\right)$ converges towards zero in $\mathbf{Z}_{p}$ as $n$ goes to $\infty$. By the Bell-Poonen theorem (Theorem 2.4), each $f_{i}:=\Phi\left(\gamma_{i}\right)$ gives rise to a flow $t \mapsto f_{i}^{t}$, $t \in \mathbf{Z}_{p}$; moreover, $\left\|f_{i}^{t}-\mathrm{id}\right\| \leqslant p^{m}$ if $|t|<p^{m}$ (apply Lemma 2.3 and the last assertion in the Bell-Poonen theorem). Thus, the lemma follows from Lemma 2.1 and the equality

$$
\Phi\left(\alpha_{n}\right)=f_{1}^{t_{1}(n)} \ldots f_{r}^{t_{r}(n)}
$$

To prove this equality, one only needs to check it in the group $\widehat{D}$, because $D$ embeds into $\widehat{D}$. But, in $\widehat{D}$, the equality holds trivially because the homomorphism $\Gamma_{0} \rightarrow \widehat{D}$ extends to $G_{0}$ continuously (apply properties (1) and (2) above).

LEMMA 2.13. If $\left(\alpha_{m}\right)_{m \geqslant 1}$ is a sequence of elements of $\Gamma$ that converges towards an element $\alpha_{\infty}$ of $G$, then $\left(\Phi\left(\alpha_{m}\right)\right)_{m}$ converges to an element of $\operatorname{Diff}^{\mathrm{an}}(\mathcal{U})$ which depends only on $\alpha_{\infty}$.

Proof. Since $\left(\alpha_{m}\right)_{m}$ converges, $\alpha_{m} \circ \alpha_{m^{\prime}}^{-1}$ converges towards the neutral element $1_{G}$ as $m$ and $m^{\prime}$ go to $\infty$. Consequently, Lemma 2.12 shows that the sequence $\left(\Phi\left(\alpha_{m}\right)\right)_{m}$ is a Cauchy sequence in $\operatorname{Diff}^{a n}(\mathcal{U})$, and hence a convergent sequence. $\left({ }^{1}\right)$ The limit depends

$\left.{ }^{1}\right)$ Write $\Phi\left(\alpha_{m} \circ \alpha_{m^{\prime}}^{-1}\right)=\mathrm{id}+\varepsilon_{m, m^{\prime}}$, where $\varepsilon_{m, m^{\prime}}$ is equivalent to the constant map zero in $R\langle\mathbf{x}\rangle^{d}$ modulo $|p|^{k\left(m, m^{\prime}\right)}$, with $k\left(m, m^{\prime}\right)$ that goes to $\infty$ as $m$ and $m^{\prime}$ do. Then, apply Lemma 2.1. 
only on $\alpha_{\infty}$, not on the sequence $\left(\alpha_{m}\right)_{m}$ (if another sequence $\left(\alpha_{m}^{\prime}\right)_{m}$ converges toward $\alpha_{\infty}$, consider the sequence $\left.\alpha_{1}, \alpha_{1}^{\prime}, \alpha_{2}, \alpha_{2}^{\prime}, \ldots\right)$.

We can now prove Theorem 2.11. Lemmas 2.12 and 2.13 show that $\Phi$ extends, in a unique way, to a continuous homomorphism $\bar{\Phi}: G \rightarrow D$. Moreover, this extension coincides with Bell-Poonen extensions $\mathbf{Z}_{p} \rightarrow D$ along 1-parameter subgroups of $G$ generated by elements of $\Gamma$. According to Lemma 2.10, one can find $s$ elements $\gamma_{1}, \ldots, \gamma_{s}$ of $\Gamma$, with $s=\operatorname{dim}(G)$, such that the map

$$
\left(t_{1}, \ldots, t_{s}\right) \longmapsto \pi\left(t_{1}, \ldots, t_{d}\right)=\gamma_{1}^{t_{1}} \ldots \gamma_{s}^{t_{s}}
$$

determines an analytic diffeomorphism from a neighborhood of zero in $\mathbf{Z}_{p}^{s}$ to a neighborhood of the identity in $G$. By the Bell-Poonen theorem, the map

$$
\mathbf{Z}_{p}^{s} \times \mathcal{U} \ni\left(t_{1}, \ldots, t_{s}, x\right) \longmapsto \Phi\left(\gamma_{1}\right)^{t_{1}} \circ \ldots \circ \Phi\left(\gamma_{s}\right)^{t_{s}}(x)
$$

is analytic. Thus, the action of $G$ on $\mathcal{U}$ determined by $\bar{\Phi}$ is analytic. This concludes the proof of Theorem 2.11 .

\section{Good models, $p$-adic integers, and invariant polydisks}

Start with an irreducible complex variety $X$ of dimension $d$, a finitely generated group $\Gamma$, and a homomorphism $\varrho: \Gamma \rightarrow \operatorname{Bir}(X)$. First, we explain how to replace the field $\mathbf{C}$, or any algebraically closed field $\mathbf{k}$ of characteristic zero, by the ring of $p$-adic integers $\mathbf{Z}_{p}$, for some prime $p$, the variety $X$ by a variety $X_{\mathbf{Z}_{p}}$ which is defined over $\mathbf{Z}_{p}$, and the homomorphism $\varrho$ by a homomorphism into $\operatorname{Bir}\left(X_{\mathbf{Z}_{p}}\right)$.

In a second step, we look for a polydisk $\mathcal{U} \simeq \mathbf{Z}_{p}^{d}$ in $X_{\mathbf{Z}_{p}}\left(\mathbf{Q}_{p}\right)$ which is invariant under the action of $\Gamma$ in order to apply the Bell-Poonen extension theorem (Theorem 2.4) on $\mathcal{U}$. This is easy when $\varrho(\Gamma)$ is contained in Aut $(X)$, but much harder when $\Gamma$ acts by birational transformations; $\S 7$ addresses this problem.

\subsection{From complex to $p$-adic coefficients: good models}

Let $\mathbf{k}$ be an algebraically closed field of characteristic zero. Let $X=X_{\mathbf{k}}$ be a quasi-projective variety defined over $\mathbf{k}$, for instance $X=\mathbb{A}^{d}$, the affine space. Let $\Gamma$ be a subgroup of $\operatorname{Bir}\left(X_{\mathbf{k}}\right)$ with a finite, symmetric set of generators $S=\left\{\gamma_{1}, \ldots, \gamma_{s}\right\}$. 


\subsubsection{From complex to $p$-adic coefficients}

Fix an embedding of $X$ into a projective space $\mathbb{P}_{\mathbf{k}}^{N}$ and write

$$
X=\mathcal{Z}(\mathfrak{a}) \backslash \mathcal{Z}(\mathfrak{b})
$$

where $\mathfrak{a}$ and $\mathfrak{b}$ are two homogeneous ideals in $\mathbf{k}\left[\mathbf{x}_{0}, \ldots, \mathbf{x}_{N}\right]$ and $\mathcal{Z}(\mathfrak{a})$ denotes the zero-set of the ideal $\mathfrak{a}$. Choose generators $\left(F_{i}\right)_{i=1}^{a}$ and $\left(G_{j}\right)_{j=1}^{b}$ for $\mathfrak{a}$ and $\mathfrak{b}$, respectively.

Let $C$ be a finitely generated $\mathbf{Q}$-algebra containing the set $B$ of all coefficients of the $F_{i}$, the $G_{j}$, and the polynomial formulas defining the generators $\gamma_{k} \in S$; more precisely, each $\gamma_{k}$ is defined by ratios of regular functions on affine open subsets $\mathcal{V}_{l}=X \backslash W_{l}$, and one includes the coefficients of the formulas for these regular functions and for the defining equations of the Zariski closed subsets $W_{l}$. One can view $X$ and $\Gamma$ as defined over $\operatorname{Spec}(C)$.

Lemma 3.1. (See [48, $\S 4$ and $\S 5]$ and [6, Lemma 3.1]) Let $L$ be a finitely generated extension of $\mathbf{Q}$ and $B$ be a finite subset of $L$. The set of primes $p$ for which there exists an embedding of $L$ into $\mathbf{Q}_{p}$ that maps $B$ into $\mathbf{Z}_{p}$ has positive density among the set of all primes.

By positive density, we mean that there exist $\varepsilon>0$ and $N_{0}>0$ such that, among the first $N$ primes, the proportion of primes $p$ that satisfy the statement is bounded from below by $\varepsilon$ if $N \geqslant N_{0}$.

Apply this lemma to the fraction field $L=\operatorname{Frac}(C)$ and the set $B$ of coefficients. This provides an odd prime $p$ and an embedding $\iota: L \rightarrow \mathbf{Q}_{p}$ with $\iota(B) \subset \mathbf{Z}_{p}$. Applying $\iota$ to the coefficients of the formulas that define $X$ and the elements of $\Gamma$, we obtain what will be called a "model of the pair $(X, \Gamma)$ over $\mathbf{Z}_{p}$ "; in particular, $\Gamma$ embeds into $\operatorname{Bir}\left(X_{\mathbf{Z}_{p}}\right)$, or in $\operatorname{Aut}\left(X_{\mathbf{Z}_{p}}\right)$ if $\Gamma$ is initially a subgroup of $\operatorname{Aut}\left(X_{\mathbf{k}}\right)$. The following paragraphs clarify this idea.

\subsubsection{Good models}

Let $R$ be an integral domain. Let $X_{R}$ and $Y_{R}$ be separated and reduced schemes of finite type defined over $R$. Assume, moreover, that the morphism $X_{R} \rightarrow \operatorname{Spec}(R)$ is dominant on every irreducible component of $X_{R}$. Let $\mathcal{U}$ and $\mathcal{V}$ be two dense open subsets of $X_{R}$. Two morphisms of $R$-schemes $f: \mathcal{U} \rightarrow Y_{R}$ and $g: \mathcal{V} \rightarrow Y_{R}$ are equivalent if they coincide on some dense open subset $W$ of $U \cap V$; rational maps $f: X_{R^{--} \rightarrow Y_{R}}$ are equivalence classes for this relation $([38, \S 7.1])$. For any rational map $f$, there is a maximal open subset $\operatorname{Dom}(f) \subset X_{R}$ on which $f$ induces a morphism: if a morphism $\mathcal{V} \rightarrow Y_{R}$ is in the equivalence 
class of $f$, then $\mathcal{V}$ is contained in $\operatorname{Dom}(f)$. This open subset $\operatorname{Dom}(f)$ is the domain of definition of $f([38, \S 7.2])$; its complement is the indeterminacy locus $\operatorname{Ind}(f)$.

A rational map $f$ is birational if there is a rational map $g: Y_{R^{--} \rightarrow X_{R}}$ such that $g \circ f=\mathrm{ld}$ and $f \circ g=\mathrm{ld}$. The group of birational transformations $f: X_{R^{--} \rightarrow X_{R}}$ is denoted $\operatorname{Bir}\left(X_{R}\right)$; the group of regular automorphisms is denoted $\operatorname{Aut}\left(X_{R}\right)$. Consider a birational map $f: X \rightarrow Y$ and denote by $g$ the inverse map $f^{-1}$. The domains of definition $\operatorname{Dom}(f)$ and $\operatorname{Dom}(g)$ are dense open subsets of $X$ and $Y$, respectively, for the Zariski topology. Then, set $U_{R, f}=\left(\left.f\right|_{\operatorname{Dom}(f)}\right)^{-1}(\operatorname{Dom}(g))$. As $f$ is birational, $U_{R, f}$ is open and dense in $X$. The restriction of $f$ to this open subset is an open immersion of $U_{R, f}$ into $Y$; indeed, $f\left(U_{R, f}\right)$ is the open set $\left(\left.g\right|_{\operatorname{Dom}(g)}\right)^{-1}\left(U_{R, f}\right)$ and $g$ is a morphism on $f\left(U_{R, f}\right)$ such that $g \circ f=\mathrm{ld}$. Moreover, $U_{R, f}$ is the largest open subset of $X$ on which $f$ is locally, for the Zariski topology, an open immersion. In what follows, we denote by $B_{R, f}$ the complement of $U_{R, f}$ in $X_{R}$ : this nowhere dense Zariski closed subset is the set of bad points; on its complement, $f$ is an open immersion.

For $y \in \operatorname{Spec}(R)$, denote by $X_{y}$ the reduced fiber of $X_{R}$ above $y$. If $X_{y} \cap B_{R, f}$ is nowhere dense in $X_{y}$, then $f$ induces a birational transformation $f_{y}: X_{y^{--} \rightarrow X_{y}}$; we have $\operatorname{Ind}\left(f_{y}\right) \subset X_{y} \cap B_{R, f}$, and this inclusion may be strict.

If $\eta$ is the generic point of $\operatorname{Spec}(R)$, we can always restrict $f$ to $X_{\eta}$. This map $f \mapsto f_{\eta}$ determines an isomorphism of groups $i$ : $\operatorname{Bir}\left(X_{R}\right) \rightarrow \operatorname{Bir}\left(X_{\eta}\right)$. More precisely, the following holds: let $K$ be the fraction field of the integral domain $R$; let $X_{R}$ be a separated and reduced scheme over $R$; assume that $X_{R}$ is of finite type over $R$, and that the morphism $X_{R} \rightarrow \operatorname{Spec}(R)$ is dominant on every irreducible component of $X_{R}$; then, the map $i$ : $\operatorname{Bir}\left(X_{R}\right) \rightarrow \operatorname{Bir}\left(X_{K}\right)$ is bijective. Indeed, $i$ is injective because $X_{R}$ is of finite type over $R$ and the map $X_{R} \rightarrow \operatorname{Spec}(R)$ is dominant on every irreducible component of $X_{R}$. It is surjective, because $f_{\eta}$ can be defined on a dense affine open subset

$$
U=\operatorname{Spec}\left(R\left[x_{1}, \ldots, x_{m}\right] / I\right)
$$

of $X_{R}$ by polynomial functions $G_{i}$ with coefficients in $K$. There is an element $d$ of $R$ such that the functions $d G_{i}$ have coefficients in $R$; then, $f_{\eta}$ extends as a morphism on $\left.\operatorname{Spec}\left(R\left[1 / d, x_{1}, \ldots, x_{m}\right] / I\right)\right)$. (See $[18, \S 9.1]$ for details.)

Let $\mathbf{k}$ be an algebraically closed field of characteristic zero. Let $X_{\mathbf{k}}$ be an irreducible variety which is defined over $\mathbf{k}$. Let $\Gamma$ be a subgroup of $\operatorname{Bir}\left(X_{\mathbf{k}}\right)\left(\operatorname{resp}\right.$. Aut $\left.\left(X_{\mathbf{k}}\right)\right)$. Let $R$ be a subring of $\mathbf{k}$. We say that the pair $(X, \Gamma)$ is defined over $R$ if there is a separated, reduced, irreducible scheme $X_{R}$ over $R$ for which the structure morphism $X_{R} \rightarrow \operatorname{Spec}(R)$ is dominant, and an embedding $\Gamma \rightarrow \operatorname{Bir}\left(X_{R}\right)$ (resp. in Aut $\left(X_{R}\right)$ ) such that both $X_{\mathbf{k}}$ and $\Gamma$ are obtained from $X_{R}$ by base change: $X_{\mathbf{k}}=X_{R} \times_{\operatorname{Spec}(R)} \operatorname{Spec}(\mathbf{k})$ and similarly for all elements $f \in \Gamma$. 
Let $p$ be a prime number. A model for the pair $(X, \Gamma)$ over the ring $\mathbf{Z}_{p}$ is given by the following data. First, a ring $R \subset \mathbf{k}$ on which $X$ and $\Gamma$ are defined, and an embedding $\iota: R \rightarrow \mathbf{Z}_{p}$. Then, an irreducible scheme $X_{\mathbf{Z}_{p}}$ over $\mathbf{Z}_{p}$ and an embedding $\varrho: \Gamma \rightarrow \operatorname{Bir}\left(X_{\mathbf{Z}_{p}}\right)$ (resp. in Aut $\left(X_{\mathbf{Z}_{p}}\right)$ ) such that

(i) $X_{\mathbf{Z}_{p}} \simeq X_{R} \times_{\operatorname{Spec}(R)} \operatorname{Spec}\left(\mathbf{Z}_{p}\right)$ is the base change of $X_{R}$ and $\varrho(f)$ is the base change of $f \in \operatorname{Bir}\left(X_{R}\right)$ for every $f$ in $\Gamma$.

A good model for the pair $(X, \Gamma)$ over the ring $\mathbf{Z}_{p}$ is a model such that

(ii) the special fiber $X_{\mathbf{F}_{p}}$ of $X_{\mathbf{Z}_{p}} \rightarrow \operatorname{Spec}\left(\mathbf{Z}_{p}\right)$ is absolutely reduced and irreducible and its dimension is

$$
\operatorname{dim}_{\mathbf{F}_{p}}\left(X_{\mathbf{F}_{p}}\right)=\operatorname{dim}_{\mathbf{Q}_{p}}\left(X_{\mathbf{Z}_{p}} \times_{\operatorname{Spec}(R)} \operatorname{Spec}\left(\mathbf{Q}_{p}\right)\right) ;
$$

(iii) for all $f \in \Gamma$, the special fiber $X_{\mathbf{F}_{p}}$ is not contained in $B_{\mathbf{Z}_{p}, \varrho(f)}$.

If $K$ is a finite extension of $\mathbf{Q}_{p}$ and $\mathcal{O}_{K}$ is its valuation ring, one can also introduce the notion of good models over $\mathcal{O}_{K}$. The following is proven in the appendix.

Proposition 3.2. Let $X$ be an irreducible complex projective variety, and $\Gamma$ be a finitely generated subgroup of $\operatorname{Bir}(X)$ (resp. of Aut $(X))$. Then, there exist infinitely many primes $p \geqslant 3$ such that the pair $(X, \Gamma)$ has a good model over $\mathbf{Z}_{p}$.

\subsection{From birational transformations to local analytic diffeomorphisms}

\subsubsection{Automorphisms and invariant polydisks}

Now, for simplicity, assume that $X$ is the affine space $\mathbb{A}^{d}$. Let $p$ be an odd prime number, and let $\Gamma$ be a subgroup of $\operatorname{Aut}\left(\mathbb{A}_{\mathbf{Z}_{p}}^{d}\right)$; all elements of $\Gamma$ are polynomial automorphisms of the affine space defined by formulas with coefficients in $\mathbf{Z}_{p}$. Reduction modulo $p$ provides a homomorphism from $\Gamma$ to the group $\operatorname{Aut}\left(\mathbb{A}_{\mathbf{F}_{p}}^{d}\right)$ : every automorphism $f \in \Gamma$ determines an automorphism $\bar{f}$ of the affine space with coefficients in $\mathbf{F}_{p}$. One can also reduce modulo $p^{2}, p^{3}, \ldots$.

If $R_{0}$ is a finite ring, then $\mathbb{A}^{d}\left(R_{0}\right)$ and $\mathrm{GL}_{d}\left(R_{0}\right)$ are both finite. Therefore, the automorphisms $f \in \Gamma$ with $f(m)=m\left(\bmod p^{2}\right)$ and $d f_{m}=\operatorname{Id}(\bmod p)$ for all points $m$ in $\mathbb{A}^{d}\left(\mathbf{Z}_{p}\right)$ form a finite-index subgroup $\Gamma_{0}$ of $\Gamma$. Every element of $\Gamma_{0}$ can be written

$$
f(\mathbf{x})=p^{2} A_{0}+\left(\mathrm{Id}+p B_{1}\right)(\mathbf{x})+\sum_{k \geqslant 2} A_{k}(\mathbf{x}),
$$

where $A_{0}$ is a point with coordinates in $\mathbf{Z}_{p}, B_{1}$ is a $d \times d$ matrix with coefficients in $\mathbf{Z}_{p}$, and $\sum_{k \geqslant 2} A_{k}(\mathbf{x})$ is a finite sum of higher-degree homogeneous terms with coefficients in $\mathbf{Z}_{p}$. Rescaling, one gets

$$
p^{-1} f(p \mathbf{x})=p A_{0}+\left(\operatorname{Id}+p B_{1}\right)(\mathbf{x})+\sum_{k \geqslant 2} p^{k-1} A_{k}(\mathbf{x}) .
$$


Thus, the Bell-Poonen extension theorem (Theorem 2.4) applies to $p^{-1} f(p \mathbf{x})$, because $p \geqslant 3$.

A similar argument applies to automorphism groups $\Gamma$ of any quasi-projective variety $X$ of dimension $d$. One first replaces $\mathbf{Q}_{p}$ by a finite extension $K$ to assure the existence of at least one point $m$ in $X\left(R / \mathbf{m}_{K}\right)$ (with $R$ the valuation ring of $K$ ). Then, the stabilizer of $m$ modulo $\mathbf{m}_{K}$ is a finite-index subgroup, because $X\left(R / \mathbf{m}_{K}\right)$ is a finite set; this group fixes a polydisk in $X(K)$ and the Bell-Poonen theorem can be applied to a smaller, finite-index subgroup. This provides the following statement, the proof of which is given in [7] (Propositions 4.4 and 2.2), when the group $\Gamma$ is cyclic. Propositions 3.2 and 3.4 are inspired by [7] and also imply this statement.

Proposition 3.3. (see [7]) Let $X_{\mathbf{Z}_{p}}$ be a quasi-projective variety defined over $\mathbf{Z}_{p}$, and let $\Gamma$ be a subgroup of $\operatorname{Aut}\left(X_{\mathbf{Z}_{p}}\right)$. Then, there exist a finite extension $K$ of $\mathbf{Q}_{p}$, a finite-index subgroup $\Gamma_{0}$ of $\Gamma$, and an analytic diffeomorphism $\varphi$ from the unit polydisk $\mathcal{U}=R^{d} \subset K^{d}$ to an open subset $\mathcal{V}$ of $X(K)$ such that $\mathcal{V}$ is $\Gamma_{0}$-invariant and the action of $\Gamma_{0}$ on $\mathcal{V}$ is conjugate, via $\varphi$, to a subgroup of $\operatorname{Diff}^{\mathrm{an}}(\mathcal{U})_{1}$.

Combining this result with Proposition 3.2, we get: If a finitely generated group $\Gamma$ admits a faithful action by automorphisms on some irreducible $d$-dimensional complex variety, there are a finite-index subgroup $\Gamma_{0}$ in $\Gamma$, a prime $p$, and a finite extension $K$ of $\mathbf{Q}_{p}$ such that $\Gamma_{0}$ admits a faithful action by Tate analytic diffeomorphisms on a polydisk $\mathcal{U} \subset K^{d}$. This will be used as a first step in the proofs of Theorems A and D.

\subsubsection{Birational transformations and invariant polydisks}

Let us now deal with invariant polydisks for groups of birational transformations. Let $X_{\mathbf{Z}_{p}}$ be a projective variety defined over $\mathbf{Z}_{p}$ and let $\Gamma$ be a subgroup of $\operatorname{Bir}\left(X_{\mathbf{Z}_{p}}\right)$ with a finite symmetric set of generators $S$. Let $X_{\mathbf{F}_{p}}$ be the special fiber of $X_{\mathbf{Z}_{p}}$. We assume that the special fiber is not contained in $B_{\mathbf{Z}_{p}, s}$ for any $s \in S$; this implies that $X_{\mathbf{F}_{p}}$ is not contained in $B_{\mathbf{Z}_{p}, g}$ for every $g \in \Gamma$. By restriction, we obtain a homomorphism $\Gamma \rightarrow \operatorname{Bir}\left(X_{\mathbf{F}_{p}}\right)$. These assumptions are satisfied by good models.

Let $K$ be a finite extension of $\mathbf{Q}_{p}, O_{K}$ be the valuation ring of $K$, and $\mathbf{F}$ be the residue field of $O_{K}$; by definition, $\mathbf{F}=O_{K} / \mathbf{m}_{K}$, where $\mathbf{m}_{K}$ is the maximal ideal of $O_{K}$. Denote by $|\cdot|_{p}$ the $p$-adic norm on $K$, normalized by $|p|_{p}=1 / p$. Set

$$
X_{O_{K}}=X_{\mathbf{Z}_{p}} \times_{\operatorname{Spec}\left(\mathbf{Z}_{p}\right)} \operatorname{Spec}\left(O_{K}\right)
$$

The generic fiber

$$
X_{\mathbf{Z}_{p}} \times \operatorname{Spec}\left(\mathbf{Z}_{p}\right) \operatorname{Spec}(K)
$$


is denoted by $X_{K}$, and the special fiber is

$$
X_{\mathbf{F}}=X_{\mathbf{Z}_{p}} \times_{\operatorname{Spec}\left(\mathbf{Z}_{p}\right)} \operatorname{Spec}(\mathbf{F}) .
$$

Denote by $r: X_{K}(K) \rightarrow X_{\mathbf{F}}(\mathbf{F})=X(\mathbf{F})$ the reduction map. Let $x$ be a smooth point in $X(\mathbf{F})$ and $\mathcal{V}$ be the open subset of $X_{K}(K)$ consisting of points $z$ satisfying $r(z)=x$.

Proposition 3.4. (See also [7, Proposition 2.2]) There exists an analytic diffeomorphism $\varphi$ from the unit polydisk $\mathcal{U}=O_{K}^{d}$ to the open subset $\mathcal{V}$ of $X_{K}(K)$ such that, for every $f \in \operatorname{Bir}\left(X_{O_{K}}\right)$ with $x \notin B_{O_{K}, f}$ and $f(x)=x$, the set $\mathcal{V}$ is $f$-invariant and the action of $f$ on $\mathcal{V}$ is conjugate, via $\varphi$, to a Tate analytic diffeomorphism on $\mathcal{U}$. Thus, if $\Gamma \subset \operatorname{Bir}\left(X_{\mathbf{Z}_{p}}\right)$ satisfies

(i) $x$ is not contained in any of the sets $B_{O_{K}, f}($ for $f \in \Gamma)$,

(ii) $f(x)=x$ (for every $f \in \Gamma)$,

then $\mathcal{V}$ is $\Gamma$-invariant and $\varphi$ conjugates the action of $\Gamma$ on $\mathcal{V}$ to a group of analytic diffeormorphisms of the polydisk $\mathcal{U}$.

Thus, once a good model has been constructed, the existence of an invariant polydisk on which the action is analytic is equivalent to the existence of a smooth fixed point $x \in X_{\mathbf{F}}(\mathbf{F})$ in the complement of the bad loci $B_{O_{K}, f}, f$ in $\Gamma$. Periodic orbits correspond to polydisks which are invariant by finite-index subgroups. This will be used to prove Theorems B and C.

We shall prove this proposition in the appendix. Note that Propositions 3.4 and 3.2 , together with rescaling argument of $§ 3.2 .1$, provide a proof of Proposition 3.3.

\section{Regular actions of $\mathrm{SL}_{n}(\mathrm{Z})$ on quasi-projective varieties}

In this section, we prove the first assertion of Theorem A together with one of its corollaries. Thus, our goal is the following statement.

TheOREM 4.1. Let $n \geqslant 2$ be an integer. Let $\Gamma$ be a finite-index subgroup of $\mathrm{SL}_{n}(\mathbf{Z})$. If $\Gamma$ embeds into the group of automorphisms of a complex quasi-projective variety $X$, then $\operatorname{dim}(X) \geqslant n-1$; if $X$ is a complex affine space, then $\operatorname{dim}(X) \geqslant n$.

\subsection{Dimension 1}

When $\operatorname{dim}_{\mathbf{C}}(X)=1$, the group of automorphisms of $X$ is isomorphic to $\mathrm{PGL}_{2}(\mathbf{C})$, if $X$ is the projective line, and is virtually solvable, otherwise. On the other hand, every finiteindex subgroup of $\mathrm{SL}_{n}(\mathbf{Z})$ contains a non-abelian free group if $n \geqslant 2$ (see [39, Chapter 1]). Theorems A and 4.1 follow from these remarks when $n=2$ or $\operatorname{dim}(X)=1$. In what follows, we assume $\operatorname{dim}_{\mathbf{C}}(X) \geqslant 2$ and $n \geqslant 3$. 


\subsection{Congruence subgroups of $\mathrm{SL}_{n}(\mathrm{Z})$; see [2], [62]}

\subsubsection{Normal subgroups}

Let $\Gamma$ be a finite-index subgroup of $\mathrm{SL}_{n}(\mathbf{Z})$. For $n \geqslant 3$, the group $\Gamma$ is a lattice in the higher-rank almost simple Lie group $\mathrm{SL}_{n}(\mathbf{R})$. For such a lattice, every normal subgroup is either finite and central, or cofinite. In particular, the derived subgroup $[\Gamma, \Gamma]$ has finite index in $\Gamma$.

\subsubsection{Strong approximation}

For any $n \geqslant 2$ and $m \geqslant 1$, denote by $\Gamma_{m}$ and $\Gamma_{m}^{*}$ the following subgroups of $\operatorname{SL}_{n}(\mathbf{Z})$ :

$$
\begin{aligned}
& \Gamma_{m}=\left\{B \in \mathrm{SL}_{n}(\mathbf{Z}): B \equiv \mathrm{Id}(\bmod m)\right\} \\
& \Gamma_{m}^{*}=\left\{B \in \mathrm{SL}_{n}(\mathbf{Z}): \text { there exists } a \in \mathbf{Z} \text { such that } B \equiv a \operatorname{Id}(\bmod m)\right\} .
\end{aligned}
$$

By definition, $\Gamma_{m}$ is the principal congruence subgroup of level $m$.

Let $p$ be a prime number. The closure of $\Gamma_{m}$ in $\mathrm{SL}_{n}\left(\mathbf{Z}_{p}\right)$ is the finite-index, open subgroup of matrices which are equal to Id modulo $m$; thus, if $m=p^{u} r$ with $r \wedge p=1$, the closure of $\Gamma_{m}$ in $\mathrm{SL}_{n}\left(\mathbf{Q}_{p}\right)$ coincides with the open subgroup of matrices $M \in \mathrm{SL}_{n}\left(\mathbf{Z}_{p}\right)$ which are equal to Id modulo $p^{u}$.

The strong approximation theorem states that the image of $\mathrm{SL}_{n}(\mathbf{Z})$ is dense in the product $\prod_{p} \mathrm{SL}_{n}\left(\mathbf{Z}_{p}\right)$ (product over all prime numbers). If $\Gamma$ has finite index in $\mathrm{SL}_{n}(\mathbf{Z})$, its closure in $\prod_{p} \mathrm{SL}_{n}\left(\mathbf{Z}_{p}\right)$ is a finite-index subgroup; it contains almost all $\mathrm{SL}_{n}\left(\mathbf{Z}_{p}\right)$.

\subsubsection{Congruence subgroup property}

A deep property that we shall use is the congruence subgroup property, which holds for $n \geqslant 3$. It asserts that every finite-index subgroup $\Gamma$ of $\mathrm{SL}_{n}(\mathbf{Z})$ contains a principal congruence subgroup $\Gamma_{m}$; if $\Gamma$ is normal, there exists a unique integer $m$ with $\Gamma_{m} \subset \Gamma \subset \Gamma_{m}^{*}$. We shall come back to this property in $\S 8.1$ for more general algebraic groups (the congruence subgroup property is not known in full generality for cocompact lattices).

Another way to state the congruence subgroup and strong approximation properties is to say that the profinite completion of $\mathrm{SL}_{n}(\mathbf{Z})$ coincides with the product $\prod_{p} \mathrm{SL}_{n}\left(\mathbf{Z}_{p}\right)$. If $\Gamma$ has finite index in $\mathrm{SL}_{n}(\mathbf{Z})$, its profinite completion is a product $\prod_{q} G_{q} \subset \prod_{q} \mathrm{SL}_{n}\left(\mathbf{Z}_{q}\right)$, where each $G_{q}$ has finite index in $\mathrm{SL}_{n}\left(\mathbf{Z}_{q}\right)$, and $G_{q}=\mathrm{SL}_{n}\left(\mathbf{Z}_{q}\right)$ for almost all primes $q$.

Remark 4.2. Fix $n \geqslant 3$. For every prime number $q$, the group $\mathrm{SL}_{n}\left(\mathbf{Z}_{q}\right)$ is a perfect group, because it is generated by the elementary matrices $e_{i j}(r), r \in \mathbf{Z}_{q}$, and every elementary matrix is a commutator. Thus, every homomorphism from $\mathrm{SL}_{n}\left(\mathbf{Z}_{q}\right)$ to a $p$-group 
is trivial, because every $p$-group is nilpotent. Thus, the pro- $p$ completion of $\mathrm{SL}_{n}\left(\mathbf{Z}_{q}\right)$ is trivial.

Before stating the following lemma, recall that the concept of virtual pro- $p$ completion is introduced in $§ 2.4 .2$.

LEMMA 4.3. Let $n$ be an integer $\geqslant 3$. Let $\Gamma$ be a finite-index subgroup of $\mathrm{SL}_{n}(\mathbf{Z})$. Let $\Gamma_{m}$ be a principal congruence subgroup contained in $\Gamma$. If $p$ divides the integer $m$, the pro-p completion of $\Gamma_{m}$ coincides with its closure in $\mathrm{SL}_{n}\left(\mathbf{Z}_{p}\right)$. Therefore, $\mathrm{SL}_{n}\left(\mathbf{Z}_{p}\right)$ is a virtual pro-p completion of its subgroup $\Gamma$.

Proof. Fix a positive integer $m$ such that $\Gamma$ contains $\Gamma_{m}$ and $p$ divides $m$. The profinite completion of $\Gamma_{m}$ coincides with the product $\prod_{q} G_{q}$, where $G_{q}$ is the closure of $\Gamma_{m}$ in $\mathrm{SL}_{n}\left(\mathbf{Z}_{q}\right)$. If $m=p^{u} r$ with $p \wedge r=1$, then $G_{p}$ is the open, pro- $p$ subgroup of $\operatorname{SL}_{n}\left(\mathbf{Z}_{p}\right)$ defined by $G_{p}=\left\{B \in \mathrm{SL}_{n}\left(\mathbf{Z}_{p}\right): B \equiv \operatorname{Id}\left(\bmod p^{u}\right)\right\}$. If $q$ does not divide $m, G_{q}$ is equal to $\mathrm{SL}_{n}\left(\mathbf{Z}_{q}\right)$. If $q \neq p$ and $q$ divides $m$, then the group $G_{q}$ is an open subgroup of the pro- $q$ group $\left\{B \in \mathrm{SL}_{n}\left(\mathbf{Z}_{q}\right): B \equiv \operatorname{Id}(\bmod q)\right\}$. Thus, if $q \neq p$, then the pro- $p$ completion of $G_{q}$ is trivial; and the pro- $p$ completion of $\Gamma_{m}$ coincides with its closure $G_{p}$ in $\mathrm{SL}_{n}\left(\mathbf{Z}_{p}\right)$.

\subsection{Extension, algebraic groups, and Lie algebras}

Given an analytic diffeomorphism $f$ of the unit polydisk $\mathcal{U}$, its jacobian determinant is an analytic function which is defined by $\operatorname{Jac}(f)(\mathbf{x})=\operatorname{det}\left(d f_{\mathbf{x}}\right)$, where $d f_{\mathbf{x}}$ denotes the differential of $f$ at $\mathbf{x}$. One says that the jacobian determinant of $f$ is identically equal to 1 if $\operatorname{Jac}(f)$ is the constant function 1. In the following theorem, $p$ is an odd prime, and $K$ and $R$ are as in $\S 2.1 .1$.

THEOREM 4.4. Let $n \geqslant 3$ be an integer. Let $\Gamma$ be a finite-index subgroup of $\mathrm{SL}_{n}(\mathbf{Z})$. Let $\mathcal{U}$ be the unit polydisk $R^{d}$, for some $d \geqslant 1$. Let $\Phi: \Gamma \rightarrow \operatorname{Diff}^{\text {an }}(\mathcal{U})$ be a homomorphism such that $f(\mathbf{x}) \equiv \mathbf{x}(\bmod p)$ for all $f$ in $\Phi(\Gamma)$. If the image of $\Phi$ is infinite, then $n-1 \leqslant d$. If, moreover, the jacobian determinant is identically equal to 1 for all $f$ in $\Phi(\Gamma)$, then $n \leqslant d$.

Remark 4.5. All proper subalgebras of $\mathfrak{s l}_{n}\left(\mathbf{Q}_{p}\right)$ have codimension $\geqslant n-1$, and there are two conjugacy classes of algebraic subgroups of codimension $n-1$ in $\mathrm{SL}_{n, \mathbf{Q}_{p}}$ for $n \geqslant 3$. The stabilizer of a point in the projective space $\mathbb{P}^{n-1}\left(\mathbf{Q}_{p}\right)$, and the stabilizer of a hyperplane in that space (see [9, Chapter 5$]$ and $\S 8.2$ below). These conjugacy classes are exchanged by the outer automorphism $\theta: A \mapsto{ }^{t} A^{-1}$. When $n=2, \theta$ is an inner automorphism and there is only one conjugacy class. 
Proof. According to Lemma 4.3 and Theorem 2.11, $\mathrm{SL}_{n}\left(\mathbf{Z}_{p}\right)$ is a virtual pro- $p$ completion of $\Gamma$, and there is a principal congruence subgroup $\Gamma_{m} \subset \Gamma$ such that $\Phi$ extends as an analytic homomorphism $\bar{\Phi}: G \rightarrow \operatorname{Diff}^{\text {an }}(\mathcal{U})_{1}$ from the group $\Gamma_{m}$ to its pro- $p$ completion $G=\bar{\Gamma}_{m} \subset \mathrm{SL}_{n}\left(\mathbf{Z}_{p}\right)$. The differential $d \bar{\Phi}_{\text {Id }}$ provides a homomorphism of Lie algebras

$$
d \bar{\Phi}_{\mathrm{Id}}: \mathfrak{s l}_{n}\left(\mathbf{Q}_{p}\right) \longrightarrow \Theta(\mathcal{U}),
$$

where $\Theta(\mathcal{U})$ is the algebra of analytic vector fields on $\mathcal{U}$. If the image of $\Phi$ is infinite, its kernel is a finite central subgroup of $\Gamma$ (see $\S 4.2)$; hence, there are infinite-order elements in $\Phi(\Gamma)$. The vector field corresponding to such an element does not vanish identically; thus, $d \bar{\Phi}_{\text {Id }}$ is a non-trivial homomorphism. Since $\mathfrak{s l}_{n}\left(\mathbf{Q}_{p}\right)$ is a simple Lie algebra, $d \bar{\Phi}_{\text {Id }}$ is an embedding. Pick $w$ in $\mathfrak{s l}_{n}\left(\mathbf{Q}_{p}\right) \backslash\{0\}$. Since $d \bar{\Phi}_{\text {Id }}$ is an embedding, there is a point $o$ in $\mathcal{U}$ such that $d \bar{\Phi}_{\operatorname{Id}}(w)(o) \neq 0$. The subset of elements $v \in \mathfrak{s l}_{n}\left(\mathbf{Q}_{p}\right)$ such that $d \bar{\Phi}_{\operatorname{Id}}(v)(o)=0$ constitutes a proper subalgebra $\mathfrak{p}_{\Phi}$ of $\mathfrak{s l}_{n}\left(\mathbf{Q}_{p}\right)$ of codimension at most $d$. Thus, $d \geqslant n-1$ by Remark 4.5.

Let us now assume that $d=n-1$. Consider the parabolic subgroup $P_{0}$ of $\mathrm{SL}_{n}$ which is defined as the stabilizer of the point $m_{0}=[1: 0: 0 \ldots: 0]$ in the projective space $\mathbb{P}^{n-1}$. Assume, first, that $\mathfrak{p}_{\Phi}$ coincides with the Lie algebra $\mathfrak{p}_{0}$ of $P_{0}$. The quotient of $\mathfrak{s l}_{n}$ by $\mathfrak{p}_{0}$ can be identified with the tangent space $T_{m_{0}} \mathbb{P}^{n-1}$ of $\mathbb{P}^{n-1}$ at $m_{0}$, and to the tangent space of $\mathcal{U}$ at the fixed point $o$. The group $P_{0}$ contains the diagonal matrices with diagonal coefficients $a_{11}=a$ and $a_{i i}=b$ for $2 \leqslant i \leqslant n$, where $a$ and $b$ satisfy the relation $a b^{n-1}=1$, and those diagonal matrices act by multiplication by $b / a$ on $T_{m_{0}} \mathbb{P}^{n-1}$. Thus, there are elements $g$ in $G$ fixing the point $o$ in $\mathcal{U}$ and acting by non-trivial scalar multiplications on the tangent space $T_{0} \mathcal{U}$; such elements have jacobian determinant $\neq 1$. Since $\Gamma$ is dense in $G$, and both $\Phi$ and $\operatorname{Jac}$ are continuous, there are elements $f$ in $\Gamma$ with $\operatorname{Jac}(f) \neq 1$. This concludes the proof of the theorem when $\mathfrak{p}_{\Phi}=\mathfrak{p}_{0}$, or more generally when $\mathfrak{p}_{\Phi}$ is conjugate to $\mathfrak{p}_{0}$. If $\mathfrak{p}_{\Phi}$ is not conjugate to $\mathfrak{p}_{0}$, we replace $\Phi$ by $\Phi \circ \theta$ and apply Remark 4.5 to conclude (note that the outer automorphism $\theta$ preserves $\Gamma_{m}$ and induces an analytic automorphism of $G$ ).

\subsection{Embeddings of $\mathrm{SL}_{n}(\mathrm{Z})$ in $\operatorname{Aut}(X)$ or $\operatorname{Aut}\left(\mathbb{A}_{\mathrm{C}}^{d}\right)$}

We may now prove Theorem 4.1. According to $\S 4.1$, we assume $n \geqslant 3$. Let $d$ be the dimension of $X$ and $\Psi: \Gamma \rightarrow \operatorname{Aut}(X)$ be a homomorphism with infinite image.

According to $\S 3.1$ and Proposition 3.3, one can find a prime $p \geqslant 3$, a model of $(X, \Gamma)$ over a finite extension $K$ of $\mathbf{Q}_{p}$, a finite-index subgroup $\Gamma^{\prime}$ of $\Gamma$, and a polydisk $\mathcal{U} \simeq R^{d}$ in $X(K)$ such that $\mathcal{U}$ is invariant under the action of $\Gamma^{\prime}$ and the action of $\Gamma^{\prime}$ on $\mathcal{U}$ is given by a homomorphism $\Phi: \Gamma^{\prime} \rightarrow \operatorname{Diff}^{a n}(\mathcal{U})_{1}$. Theorem 4.4 implies $\operatorname{dim}(X) \geqslant n-1$. 
Assume now that $X$ is the affine space $\mathbb{A}_{\mathbf{C}}^{d}$. If $f$ is an automorphism of $\mathbb{A}_{\mathbf{C}}^{d}$, its jacobian determinant $\operatorname{Jac}(f)$ is constant because $\operatorname{Jac}(f)$ is a polynomial function on $\mathbb{A}^{d}(\mathbf{C})$ that does not vanish. Thus, $\operatorname{Jac}(\cdot)$ provides a homomorphism from $\Gamma$ to $\left(\mathbf{C}^{*}, \cdot\right)$; since the derived group $[\Gamma, \Gamma]$ has finite index in $\Gamma$ (see $\S 4.2 .1$ ), one may assume that $\operatorname{Jac}(\Phi(\gamma))=1$ for all $\gamma \in \Gamma^{\prime}$. Then, Theorem 4.4 implies that $d \geqslant n$.

\section{Actions of $\mathrm{SL}_{n}(\mathrm{Z})$ in dimension $n-1$}

In this paragraph, we pursue the study of algebraic actions of finite-index subgroups of $\mathrm{SL}_{n}(\mathbf{Z})$ on quasi-projective varieties $X$ of dimension $d$, and complete the proof of Theorem A. The notation and main properties are the same as in $\S 4$, but with two differences: we study both regular and birational actions, and we add a constraint on the dimension of $X$, which corresponds to the limit case in the inequality $d \geqslant n-1$ of Theorem 4.1. Thus,

(i) $\Gamma$ is a finite-index subgroup of $\mathrm{SL}_{n}(\mathbf{Z})$;

(ii) $X_{\mathbf{C}}$ is a complex, irreducible, quasi-projective variety of dimension $d=n-1$;

(iii) $\Gamma$ embeds into $\operatorname{Aut}\left(X_{\mathbf{C}}\right)$ (resp. in $\operatorname{Bir}\left(X_{\mathbf{C}}\right)$ );

(iv) there is a finite extension $K$ of $\mathbf{Q}_{p}$, and a model of $(X, \Gamma)$ over the valuation ring $R$ of $K$, together with a polydisk $\mathcal{U}$ in $X(K)$ which is $\Gamma$ invariant, and on which $\Gamma$ acts by analytic diffeomorphisms, as in Proposition 3.3: this gives a homomorphism $\Phi: \Gamma \rightarrow \operatorname{Diff}^{\text {an }}(\mathcal{U})_{1}$.

THEOREM 5.1. Under the above four hypotheses (i)-(iv), there exists an isomorphism $\tau: X \rightarrow \mathbb{P}_{\mathbf{C}}^{d}$ (resp. a birational map $\tau: X-\rightarrow \mathbb{P}_{\mathbf{C}}^{d}$ ), from $X$ to the projective space of dimension $d=n-1$ and a homomorphism $\varrho: \Gamma \rightarrow \mathrm{PGL}_{n}(\mathbf{C})$ such that $\tau \circ \gamma=\varrho(\gamma) \circ \tau$ for every $\gamma$ in $\Gamma$.

Theorem A follows from Theorem 4.1, Proposition 3.3, and Theorem 5.1.

Remark 5.2. When $\Gamma$ acts by birational transformations on $X$, the existence of a $\Gamma$ invariant polydisk $\mathcal{U}$ in $X(K)$ on which $\Gamma$ acts by analytic diffeomorphisms (in particular, $\mathcal{U}$ does not contain any indeterminacy point of $\Gamma$ ) may look as a strong hypothesis. We shall obtain such polydisks in $\S 7.3$

\subsection{Extension}

We apply Lemma 4.3 and Theorem 2.11 to the analytic action of $\Gamma$ on $\mathcal{U}$. Thus, there exists a principal congruence subgroup $\Gamma_{m} \subset \Gamma$ such that the homomorphism $\Phi$ from $\Gamma_{m}$ 
to $\operatorname{Diff}^{\text {an }}(\mathcal{U})_{1}$ extends as an analytic homomorphism $\bar{\Phi}$ from the $p$-adic analytic group $G=\bar{\Gamma}_{m} \subset \mathrm{SL}_{n}\left(\mathbf{Z}_{p}\right)$ to $\operatorname{Diff}^{\text {an }}(\mathcal{U})_{1}$.

\subsection{Stabilizer of the origin in $\mathcal{U}$}

Let $P_{0}$ be the subgroup of $\mathrm{SL}_{n}\left(\mathbf{Q}_{p}\right)$ which fixes the point $m_{0}=[1: 0: \ldots: 0]$ in the projective space $\mathbb{P}^{n-1}\left(\mathbf{Q}_{p}\right)$; it is a maximal parabolic subgroup of $\mathrm{SL}_{n}\left(\mathbf{Q}_{p}\right)$. We denote by $\mathfrak{p}_{0}$ its Lie algebra, as in the proof of Theorem 4.4.

Lemma 5.3. There are an element $A$ in $\mathrm{SL}_{n}(\mathbf{Z})$ and a point $o^{\prime}$ in $\mathcal{U}$ with the following property. For the homomorphism $\bar{\Phi} \circ c_{A}: G \rightarrow \operatorname{Diff}^{a n}(\mathcal{U})_{1}$, where $c_{A}$ is either the conjugacy $c_{A}(M)=A M A^{-1}$, or its composition with the outer automorphism $\theta: M \mapsto{ }^{t} M^{-1}$, the stabilizer $P^{\prime} \subset G$ of the point $o^{\prime}$ coincides with $P_{0} \cap G$.

Let $P \subset G$ be the stabilizer of the origin $o \in \mathcal{U}$. Since $d=n-1$, the Lie algebra $\mathfrak{p}$ of $P$ has codimension $n-1$ in $\mathfrak{g}=\mathfrak{s l}_{n}\left(\mathbf{Q}_{p}\right)$, and is therefore maximal. Let $\widetilde{P}$ be the Zariski closure of $P$ in $\mathrm{SL}_{n}\left(\mathbf{Q}_{p}\right)$. Then, $\widetilde{P} \cap G$ coincides with $P$, and $\widetilde{P}$ is conjugate to $P_{0}$ or to $\theta\left(P_{0}\right)$ in $\mathrm{SL}_{n}\left(\mathbf{Q}_{p}\right)$ (see Remark 4.5). For simplicity, we assume that $\widetilde{P}$ is conjugate to $P_{0}$; if $\widetilde{P}$ is conjugate to $\theta\left(P_{0}\right)$, one only needs to replace the action of $\mathrm{SL}_{n}\left(\mathbf{Q}_{p}\right)$ on the projective space by the dual action on the space of hyperplanes in $\mathbb{P}^{n-1}\left(\mathbf{Q}_{p}\right)$, or to compose $\bar{\Phi}$ with $\theta$.

To prove the lemma, we make the following remarks.

(1) There is a point $[a]$ in $\mathbb{P}^{n-1}\left(\mathbf{Q}_{p}\right)$ such that $\widetilde{P}$ is the stabilizer of $[a]$ in $\operatorname{SL}_{n}\left(\mathbf{Q}_{p}\right)$. One can write $[a]=\left[a_{1}: \ldots: a_{n}\right]$ with $a_{i}$ in $\mathbf{Z}_{p}$ for all $1 \leqslant i \leqslant n$ and at least one $\left|a_{i}\right|$ equal to 1 .

(2) There is a matrix $B$ in $G$ such that $[B(a)]$ is in $\mathbb{P}^{n-1}(\mathbf{Z})$. Indeed, $G$ is the congruence subgroup of $\mathrm{SL}_{n}\left(\mathbf{Z}_{p}\right)$ defined as the group of matrices $M$ with $M \equiv \mathrm{Id}(\bmod m)$; if one picks an element $\left[a^{\prime}\right]=\left[a_{1}^{\prime}: \ldots: a_{n}^{\prime}\right]$ of $\mathbb{P}^{n-1}(\mathbf{Z})$ with entries $a_{i}^{\prime} \equiv a_{i}$ modulo a large power of $m$, then there is an element $B$ of $G$ that maps $[a]$ to $\left[a^{\prime}\right]$. The stabilizer of the point $o^{\prime}:=\Phi(B)(0)$ in the group $G$ is equal to $B P B^{-1}$ and coincides with the stabilizer of a point $\left[a^{\prime}\right] \in \mathbb{P}^{n-1}(\mathbf{Z})$.

(3) Then, there exists $A$ in $\mathrm{SL}_{n}(\mathbf{Z})$ such that $A\left[a^{\prime}\right]=[1: 0: \ldots: 0]$. Composing $\Phi$ with the conjugation $c_{A}$, the stabilizer of $o^{\prime}$ is now equal to $P_{0} \cap G$.

(4) Being a principal congruence subgroup, $\Gamma_{m}$ is normal in $\mathrm{SL}_{n}(\mathbf{Z})$; it is therefore invariant under the conjugacy $c_{A}: M \mapsto A M A^{-1}$ (and under the automorphism $\theta$ ). Thus, the homomorphism $\Phi \circ c_{A}\left(\right.$ resp. $\left.\bar{\Phi} \circ c_{A}\right)$ determines a new homomorphism from $\Gamma_{m}$ 
(resp. $G$ ) to $\operatorname{Diff}^{\text {an }}(\mathcal{U})_{1}$ which preserves the polydisk $\mathcal{U}$ and for which the stabilizer of $o^{\prime}$ concides with $P_{0} \cap \Gamma_{m}$ (resp. with $P_{0} \cap G$ ).

Let us now apply Lemma 5.3 to rigidify slightly the situation. We conjugate the action of $\Gamma_{m}$ on $\mathcal{U}$ by the translation $x \mapsto x+o^{\prime}$; then we compose the embedding of $\Gamma_{m}$ in Aut $(X)$ by the automorphism $c_{A}$ of $\Gamma_{m}$ given by Lemma 5.3 to assume that the stabilizer of the origin in $G$ is the intersection of $G$ with the parabolic subgroup $P_{0}$. Thus, the embedding $\Gamma_{m} \rightarrow \operatorname{Aut}(X)$ and the coordinates of $\mathcal{U}$ have been modified.

\subsection{Local normal form}

Consider the subgroup $T$ of $G$ consisting of all matrices

$$
\left(\begin{array}{cc}
1 & 0 \\
\mathbf{t} & \operatorname{ld}_{n-1}
\end{array}\right),
$$

where $\operatorname{ld}_{n-1}$ is the identity matrix of size $(n-1) \times(n-1)$ and $\mathbf{t}$ is a "vertical" vector of size $n-1$ with entries $t_{2}, \ldots, t_{n}$ in $\mathbf{Z}_{p}$ that are equal to zero modulo $m$. By construction, $T$ is contained in $G=\bar{\Gamma}_{m}$. The intersection $T \cap P_{0}$ is the trivial subgroup $\left\{\operatorname{ld}_{n}\right\}$.

The group $T$ is an abelian subgroup of $G$ of dimension $d=n-1$ that acts locally freely near the origin of $\mathcal{U}$ (if not, this would contradict the maximality of $P_{0}$ ). There are local coordinates $\mathbf{z}=\left(z_{2}, \ldots, z_{n}\right)$ on $\mathcal{U}$ near the origin $o$ such that $T$ acts by

$$
\Phi(\mathbf{t})(\mathbf{z})=\left(z_{2}+t_{2}, \ldots, z_{n}+t_{n}\right)
$$

in these coordinates, the action of the group $G$ is locally conjugate to the linear projective action of $G$ around the point $m_{0}=[1: 0: \ldots: 0]$ in $\mathbb{P}^{n-1}(K)$ (see the proof of Theorem 4.4). Note that the local coordinate $z_{i}$ may be transcendental; it is not obvious, a priori, that $z_{i}$ extends as an algebraic (or rational) function on the quasi-projective variety $X$. We shall prove that this is indeed the case in the next subsection (see Lemma 5.5).

\subsection{Invariant (algebraic) functions}

Our goal, in this subsection, is to prove Lemma 5.5. Consider the 1-parameter unipotent subgroup $E_{12}$ of $P$ whose elements have the form

$$
e_{12}(s)=\left(\begin{array}{cc}
1 & \mathbf{s} \\
0 & \operatorname{Id}_{n-1}
\end{array}\right),
$$

with $\mathbf{s}=(s, 0, \ldots, 0), s$ in $\mathbf{Z}_{p}$, and $s \equiv 0$ modulo $m$. Let $\alpha_{12}=e_{12}(s), s \in \mathbf{Z} \backslash\{0\}$, be a nontrivial element of $E_{12} \cap \Gamma_{m}$. By construction, the analytic diffeomorphism $\Phi\left(\alpha_{12}\right)$ of $\mathcal{U}$ 
transforms the local coordinate $z_{2}$ into $z_{2} /\left(1+s z_{2}\right)$, and the set $\left\{z_{2}=0\right\}$ is, locally, the set of fixed points of $\Phi\left(\alpha_{12}\right)$. Since $\Phi\left(\alpha_{12}\right)$ is the restriction to $\mathcal{U}$ of a birational transformation of $X$, the hypersurface $\left\{z_{2}=0\right\}$ is the intersection of an algebraic hypersurface of $X$ with a neighborhood of zero in $\mathcal{U}$.

Let $\alpha_{21}=e_{21}(t)$ be a non-trivial element of $T \cap \Gamma_{m}$ corresponding to the vector $\mathbf{t}=$ $(t, 0, \ldots, 0)$ (with $t \neq 0$ and $t \equiv 0$ modulo $m$ ). Then $\Phi\left(\alpha_{21}\right)^{\ell}$ acts on $\mathcal{U}$ and transports the hypersurface $\left\{z_{2}=0\right\}$ to the hypersurface $\left\{z_{2}=t \ell\right\}$. Since $\left\{z_{2}=0\right\}$ is algebraic and $\Phi\left(\alpha_{21}\right)$ is in $\operatorname{Aut}(X)$ (resp. in $\operatorname{Bir}(X)$ when the action is by birational transformations), the hypersurfaces $\left\{z_{2}=t \ell\right\}$ are all algebraic.

Denote by $T_{2}$ the subgroup of $T$ whose elements are defined by vectors of type $\mathbf{t}=\left(0, t_{3}, \ldots, t_{n}\right)$. The action of $\bar{\Phi}\left(T_{2}\right)$ on $\mathcal{U}$ preserves the local coordinate $z_{2}$ and is locally free on each level set $\left\{z_{2}=c^{s t}\right\}$. Thus, every non-trivial element of $T_{2} \cap \Gamma_{m}$ fixes infinitely many algebraic hypersurfaces in $X$, whose local equations are $z_{2}=\ell t, \ell \in \mathbf{Z}$; moreover, the orbits of $T_{2} \cap \Gamma$ are Zariski dense in these hypersurfaces.

Lemma 5.4. Let $X$ be an irreducible quasi-projective variety, defined over an algebraically closed field $\bar{K}$ of characteristic zero. Let $A$ be a group of birational transformations of $X$. If A preserves infinitely many hypersurfaces of $X$, then $A$ preserves a non-constant rational function $\varphi \in \bar{K}(X)$, meaning that $\varphi \circ a=\varphi$ for every $a \in A$.

This lemma corresponds to Theorem B of [12]: Theorem B is stated for a single transformation $g$ but applies to groups of birational transformations, as one easily checks. Let us apply it to the group $T_{2}$. From the Stein factorization theorem, we may assume that the general fibers of the function $\tau_{2}:=\varphi$ are irreducible hypersurfaces of $X$. Since the action of $T_{2}$ on the hypersurfaces $\left\{z_{2}=t \ell\right\}$ is locally free, these hypersurfaces coincide locally with the fibers of $\tau_{2}$. Thus, there is a complete curve $Y_{\bar{K}}$ and a rational function $\tau_{2}: X_{\bar{K}}^{--\rightarrow Y_{\bar{K}}}$, both defined over the algebraic closure of $K$, such that

- $\tau_{2}$ is invariant under the action of $T_{2} \cap \Gamma$, meaning that $\tau_{2} \circ \beta=\tau_{2}$ for every $\beta \in T_{2} \cap \Gamma$;

- the general hypersurface $\left\{\tau_{2}=c^{s t}\right\}$ is irreducible;

- the local analytic coordinate $z_{2}$ is, locally, a function of $\tau_{2}$ : there is an analytic 1-variable function $\phi_{2}$ such that $z_{2}=\phi_{2} \circ \tau_{2}$ on $\mathcal{U}$.

The transformation $\Phi\left(\alpha_{12}\right)$ transforms $z_{2}$ into $z_{2} /\left(1+s z_{2}\right)$ for some $s \neq 0$. Thus, it permutes the level sets of the algebraic function $\tau_{2}$. We deduce that the birational transformation $\alpha_{12}$ of $X$ induces an infinite order automorphism of $Y_{\bar{K}}$ fixing the point $\tau_{2}\left(\left\{z_{2}=0\right\}\right)$. This implies that $Y_{\bar{K}}$ is a projective line: there is an isomorphism from $Y_{\bar{K}}$ to $\mathbb{P}_{\bar{K}}^{1}$ that maps the point $\tau_{2}\left(\left\{t_{2}=0\right\}\right)$ to the point $[0: 1]$. We now fix an affine coordinate $z$ on $\mathbb{P}_{\bar{K}}^{1}$ for which this point is $z=0$.

The iterates $\Phi\left(\alpha_{12}\right)^{\ell}$ of $\Phi\left(\alpha_{12}\right)$ transform the coordinate $z_{2}$ into $z_{2} /\left(1+\ell s z_{2}\right)$. Thus, if 
$\ell=p^{n}$, one sees that the sequences of hypersurfaces $\Phi\left(\alpha_{12}^{+\ell}\right)\left(\left\{z_{2}=c\right\}\right)$ and $\Phi\left(\alpha_{12}^{-\ell}\right)\left(\left\{z_{2}=c\right\}\right)$ converge to the fixed hypersurface $\left\{z_{2}=0\right\}$ as $n$ goes to $\infty$, for every $c \in K$ with small absolute value. This implies that the automorphism of $\mathbb{P}_{\bar{K}}$ induced by $\alpha_{12}$ is a parabolic transformation, acting by

$$
z \longmapsto \frac{z}{1+s^{\prime} z}
$$

for some $s^{\prime}$. Changing the affine coordinate $z$ of $\mathbb{P}_{\bar{K}}^{1}$ into $\varepsilon z$ with $\varepsilon=s^{\prime} / s$ (hence the function $\tau_{2}$ into $\varepsilon \tau_{2}$ and $\phi_{2}(x)$ into $\phi_{2}(x / \varepsilon)$ ), one may assume that $s^{\prime}=s$. Then, both $\tau_{2}$ and $z_{2}$ satisfy the same transformation rule under $\Phi\left(\alpha_{12}\right)$ :

$$
\tau_{2} \circ \Phi\left(\alpha_{12}\right)=\frac{\tau_{2}}{1+s \tau_{2}} \quad \text { and } \quad z_{2} \circ \Phi\left(\alpha_{12}\right)=\frac{z_{2}}{1+s z_{2}} .
$$

We deduce that the function $\phi_{2}$ commutes with the linear projective transformation $z \mapsto z /(1+s z)$ :

$$
\phi_{2}\left(\frac{z}{1+\ell s z}\right)=\frac{\phi_{2}(z)}{1+\ell s \phi_{2}(z)} \quad \text { for all } \ell \in \mathbf{Z} .
$$

By construction, $\phi_{2}$ is analytic (in a neighborhood of zero) and maps zero to zero. Changing $\phi_{2}(z)$ into $\phi_{2}(z /(1+u z))$ for a well-chosen $u \neq 0$, one may assume that $\phi_{2}\left(x_{0}\right)=x_{0}$ for some $x_{0} \neq 0$. If one applies the functional equation (5.3) with $\ell \equiv 0$ modulo sufficiently large powers of $p$, then the sequence $x_{\ell}=x_{0} /\left(1+\ell s x_{0}\right)$ stays in the domain of definition of $\phi_{2}$ and $\phi_{2}\left(x_{\ell}\right)=x_{\ell}$ for all $\ell$; thus, $\phi_{2}$ is the identity: $\phi_{2}(z)=z$. In particular, the local coordinate $z_{2}$ extends to a global rational function $\tau_{2}$ on $X$.

If one applies the same strategy for $i=2,3, \ldots n$, one gets $d=n-1$ rational functions $\tau_{i}$ on $X$. These functions are local coordinates near the origin of $\mathcal{U}$. And, from $\S 5.3$, we know that these coordinates provide a local conjugacy from the action of $G$ on $\mathcal{U}$ to the linear projective action of $G$ near $[1: 0: \ldots: 0]$ in $\mathbb{P}_{K}^{n-1}$. This concludes the proof of the following lemma.

LEMmA 5.5. Each local analytic function $z_{i}, i=2, \ldots, n$, extends to a global rational function $\tau_{i} \rightarrow X_{\bar{K}} \rightarrow \bar{K}$. Altogether, they define a rational map

$$
\begin{aligned}
\tau: X_{\bar{K}} \rightarrow \mathbb{P}_{\bar{K}}^{n-1}, \\
x \longmapsto\left[1: \tau_{2}(x): \ldots: \tau_{n}(x)\right] .
\end{aligned}
$$

This rational map $\tau$ is dominant. It is equivariant with respect to the action of $\Gamma_{m}$ on $X$ and the action of $\Gamma_{m} \subset \mathrm{SL}_{n}(\mathbf{Z})$ on $\mathbb{P}_{\bar{K}}^{n-1}$ by linear projective transformations.

\subsection{Conclusion, in the case of regular actions}

We now assume that $\Gamma$ acts by automorphisms on the quasi-projective variety $X$; the case of birational transformations is dealt with in the next subsection. 


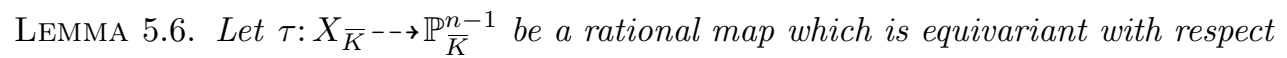
to an action of $\Gamma_{m}$ on $X_{\bar{K}}$ by automorphisms and the linear projective action of $\Gamma_{m} \subset$ $\mathrm{SL}_{n}(K)$ on $\mathbb{P}_{\bar{K}}^{n-1}$. Then, $X_{\bar{K}}$ is a projective variety, and the $\Gamma_{m}$-equivariant rational map $\tau$ is an isomorphism.

Proof. Assume that $X_{\bar{K}}$ is normal and fix a compactification $\bar{X}_{\bar{K}}$ of $X_{\bar{K}}$. Via its embedding into Aut $\left(X_{K}\right)$, the group $\Gamma_{m}$ acts by automorphisms on $X_{\bar{K}}$ and by birational transformations on $\bar{X}_{\bar{K}}$.

The image of $\Gamma_{m}$ in $\mathrm{PGL}_{n}(\bar{K})=\operatorname{Aut}\left(\mathbb{P}_{\bar{K}}^{n-1}\right)$ is a Zariski-dense subgroup $\Gamma_{m}^{\prime}$ (this is a simple instance of the Borel density theorem).

Let $\operatorname{Ind}(\tau)$ be the indeterminacy set of $\tau$. Its intersection with $X_{\bar{K}}$ is a $\Gamma_{m}$-invariant algebraic subset, because $\Gamma_{m}$ acts by automorphisms on both $X$ and $\mathbb{P}_{\bar{K}}^{n-1}$. Its total transform under $\tau$ is a $\Gamma_{m}^{\prime}$-invariant locally closed subset of $\mathbb{P}_{\bar{K}}^{n-1}$. But all such subsets are either empty or equal to $\mathbb{P}_{\bar{K}}^{n-1}$, because $\Gamma_{m}^{\prime}$ is Zariski-dense in $\operatorname{PGL}_{n}(\bar{K})$. Thus, $\operatorname{Ind}(\tau)$ does not intersect $X_{\bar{K}}$.

The image of $X_{\bar{K}}$ by $\tau$ is a constructible $\Gamma_{m}^{\prime}$-invariant subset of $\mathbb{P}_{\bar{K}}^{n-1}$; as such, it must be equal to $\mathbb{P}_{\bar{K}}^{n-1}$, because $\Gamma_{m}^{\prime}$ is Zariski-dense in $\operatorname{PGL}_{n}(\bar{K})$. Similarly, the total transform of the boundary $\bar{X}_{\bar{K}} \backslash X_{\bar{K}}$ is empty. Thus, $X_{\bar{K}}$ is complete, and $\tau$ determines a morphism from $X_{\bar{K}}$ to $\mathbb{P}_{\bar{K}}^{n-1}$. The critical locus of $\tau$ is a $\Gamma_{m}^{\prime}$-invariant subset of $\mathbb{P}_{\bar{K}}^{n-1}$ of positive codimension: it is therefore empty, and $\tau$ is an isomorphism because $\mathbb{P}_{\bar{K}}^{n-1}$ is simply connected.

If $X_{\bar{K}}$ is not normal, replace it by its normalization $\tilde{X}_{\bar{K}}$, and lift the action of $\Gamma_{m}$ on $X_{\bar{K}}$ to an action by automorphisms on $\widetilde{X}_{\bar{K}}$. We deduce that $\widetilde{X}_{\bar{K}}$ is isomorphic to the projective space and the action of $\Gamma_{m}$ on $\widetilde{X}_{\bar{K}}$ does not preserve any non-empty Zariski closed subset; thus, the normalization $\widetilde{X}_{\bar{K}} \rightarrow X_{\bar{K}}$ is an isomorphism. This proves the lemma.

Apply this lemma to the rational map $\tau$ given by Lemma 5.5. Since $X_{\bar{K}}$ is isomorphic to the projective space $\mathbb{P}_{\bar{K}}^{n-1}$, the complex variety $X_{\mathbf{C}}$ is also isomorphic to $\mathbb{P}_{\mathbf{C}}^{n-1}$. Since the action of $\Gamma$ on $X$ is an action by automorphisms, it is given by an embedding of $\Gamma$ into $\mathrm{PGL}_{n}(\mathbf{C})$. This concludes the proof of Theorem 5.1, and of Theorem A.

\subsection{Conclusion, in the case of birational actions}

Let us now assume that $X$ is projective and $\Gamma$ acts by birational transformations on $X$.

Lemma 5.7. The equivariant rational mapping $\tau: X_{\bar{K}}^{--\rightarrow} \mathbb{P}_{\bar{K}}^{n-1}$ is birational.

Proof. By construction, $\tau$ is rational and dominant; changing $X$ in a birationally equivalent variety, we assume that $X$ is normal and $\tau$ is a regular morphism. The 
elements $\gamma$ of $\Gamma_{m}$ satisfy

$$
\tau \circ \gamma_{X}=\gamma_{\mathbb{P}^{n-1}} \circ \tau
$$

where $\gamma_{X}$ denotes the birational action of $\gamma$ on $X_{\bar{K}}$ and $\gamma_{\mathbb{P}^{n-1}}$ corresponds to the linear projective action on $\mathbb{P}_{\bar{K}}^{n-1}$.

We may assume that $X$ is normal. Embed $X$ in some projective space $\mathbb{P}^{N}$, and consider the linear system of hyperplane sections $H$ of $X$. Fix an element $\gamma$ of $\Gamma_{m}$, and intersect $X$ with $n-1$ hyperplanes to get an irreducible curve $C \subset X$ that does not intersect the indeterminacy set of $\gamma$. The image of $C$ by $\gamma_{X}$ is an irreducible curve $\left(\gamma_{X}\right)_{*} C$, which satisfies $\tau_{*}\left(\left(\gamma_{X}\right)_{*}(C)\right)=\left(\gamma_{\mathbb{P}^{n-1}}\right)_{*} \tau_{*}(C)$. The degree of the curve $\left(\gamma_{\mathbb{P}^{n-1}}\right)_{*} \tau_{*}(C)$ is equal to the degree of $\tau_{*}(C)$, because $\gamma_{\mathbb{P}^{n-1}}$ is a regular automorphism of the projective space; in particular, it does not depend on $\gamma$. This implies that the degree of the curve $\left(\gamma_{X}\right)_{*} C$ in $X \subset \mathbb{P}^{N}$ is bounded by an integer $D(\tau)$ that does not depend on $\gamma$. As a consequence, the degrees of the formulas defining the elements $\gamma_{X}$ of $\Gamma_{m}$ in $\operatorname{Bir}\left(X_{\bar{K}}\right)$ are uniformly bounded. The following result shows that $\Gamma_{m}$ is "regularizable" (see [66] and the references in [15]).

Theorem 5.8. (Weil regularization theorem) Let $M$ be a projective variety, defined over an algebraically closed field. Let $\Lambda$ be a subgroup of $\operatorname{Bir}(M)$. If there is a uniform upper bound on the degrees of the elements of $\Lambda$, then there exist a birational map $\varepsilon: M-\rightarrow$ $M^{\prime}$ and a finite-index subgroup $\Lambda^{\prime}$ of $\Lambda$ such that $\varepsilon \circ \Lambda \circ \varepsilon^{-1}$ is a subgroup of $\operatorname{Aut}(M)$ and $\varepsilon \circ \Lambda^{\prime} \circ \varepsilon^{-1}$ is a subgroup of the connected component of the identity $\operatorname{Aut}(M)^{0}$ in $\operatorname{Aut}(M)$.

In our context, this result shows that, after conjugacy by a birational map $\varepsilon: X--\rightarrow$ $X^{\prime}, \Gamma_{m}$ becomes a group of automorphisms of $X^{\prime}$. Lemma 5.6 shows that the rational map $\tau \circ \varepsilon^{-1}: X_{\bar{K}}^{\prime} \rightarrow \mathbb{P}_{\bar{K}}^{n-1}$ is an isomorphism which conjugates the action of $\Gamma_{m}$ on $X_{\bar{K}}^{\prime}$ to the linear projective action on $\mathbb{P}_{\bar{K}}^{n-1}$. In particular, $\tau$ is a birational map.

Thus, $\tau$ is a birational conjugacy between the action of $\Gamma_{m}$ on $X$ and the action of $\Gamma_{m}$ by linear projective transformations on the projective space.

Lemma 5.9. The action of $\tau \circ \Gamma \circ \tau^{-1}$ on $\mathbb{P}_{\bar{K}}^{n-1}$ is an action by automorphisms.

Proof. The group $\Gamma_{m}$ is a normal, finite-index subgroup of $\Gamma$. Its image $\Gamma_{m}^{\prime}$ in $\mathrm{PGL}_{n}(\bar{K})$ is Zariski dense. Let $\gamma$ be an element of $\Gamma$, and let $\gamma_{\mathbb{P}^{n-1}}$ denote the birational transformation $\tau \circ \gamma_{X} \circ \tau^{-1}$; we have $\gamma_{\mathbb{P}^{n-1}} \Gamma_{m}^{\prime}=\Gamma_{m}^{\prime} \gamma_{\mathbb{P}^{n-1}}$. Since $\Gamma_{m}^{\prime}$ acts by automorphisms on $\mathbb{P}_{\bar{K}}^{n-1}$, it fixes the indeterminacy set of $\gamma_{\mathbb{P}^{n-1}}$, and this indeterminacy set must be empty, because $\Gamma_{m}^{\prime}$ is Zariski dense in $\mathrm{PGL}_{n}(\bar{K})$. This shows that $\gamma_{\mathbb{P}^{n-1}}$ has no indeterminacy point and that $\tau \circ \Gamma \circ \tau^{-1} \subset \mathrm{PGL}_{n}(\bar{K})$.

The existence of such a conjugacy $\tau: X_{\bar{K}}^{--\rightarrow} \mathbb{P}_{\bar{K}}^{n-1}$ implies also the existence of a 
conjugacy $X_{\mathbf{C}^{--} \rightarrow} \mathbb{P}_{\mathbf{C}}^{n-1}$ over the field of complex numbers. This concludes the proof of Theorem 5.1.

\section{Mapping class groups and nilpotent groups}

To describe another application of the $p$-adic method, we study the actions by automorphisms of the mapping class groups $\operatorname{Mod}(g)$ and of nilpotent groups.

\subsection{Mapping class groups}

Recall from $\S 1.5$ that $\mathrm{ma}(\Gamma)$ is the smallest dimension of a complex irreducible variety $X$ on which some finite-index subgroup of $\Gamma$ acts faithfully by automorphisms.

Remark 6.1. Assume that $\Gamma_{0}$ is a finite-index subgroup of $\Gamma$, and that $\Gamma_{0}$ acts faithfully on a complex irreducible variety $X$. Let us show that $\Gamma$ acts faithfully on the disjoint union of $n$ copies of $X$. Indeed, $\Gamma$ acts faithfully on the quotient $\Gamma \times_{\Gamma_{0}} X$ of $\Gamma \times X$ by the action of $\Gamma_{0}$ defined by $h \cdot(g, x)=\left(g h^{-1}, h x\right)$; and $\Gamma \times_{\Gamma_{0}} X$ is a disjoint union of $\left[\Gamma: \Gamma_{0}\right]$ copies of $X$.

Thus, $\mathrm{ma}(\Gamma)$ is bounded from below by the smallest dimension of a complex variety $X$ on which $\Gamma$ acts faithfully by regular automorphisms (the dimension of $X$ is the largest dimension of its irreducible components). The following example shows that this inequality may be strict.

Example 6.2. Consider the direct product $H$ of two non-abelian free groups $\mathbb{F}_{\ell}$ and $\mathbb{F}_{\ell^{\prime}}$. Since $\mathrm{PGL}_{2}(\mathbf{C})$ contains a free group, $H$ acts faithfully on the disjoint union of two projective lines. But $H$ does not act faithfully by automorphisms on an irreducible curve.

Theorem 6.3. (Theorem D) If $\operatorname{Mod}(g)$ acts faithfully on a (not necessarily irreducible) complex variety $X$ by automorphisms, then $\operatorname{dim}(X) \geqslant 2 g-1$. The minimal dimension $\operatorname{ma}(\operatorname{Mod}(g))$ satisfies $2 g-1 \leqslant \operatorname{ma}(\operatorname{Mod}(g)) \leqslant 6 g-6$ for all $g \geqslant 2$.

Remark 6.4. We shall need the following fact because $X$ is not assumed to be irreducible: In $\operatorname{Mod}(g)$, the intersection of two infinite normal subgroups is infinite. Indeed, if $N$ is an infinite normal subgroup of $\operatorname{Mod}(g)$, then $N$ contains two pseudo-Anosov elements $a$ and $b$ that generate a free group (see [42, Theorem 2]). If $M$ is another normal subgroup containing a pseudo-Anosov element $h$, then $[w, h]=w h w^{-1} h^{-1}$ is an element of $N \cap M$ for every $w \in N$. By a theorem of McCarthy [52], the centralizer of a pseudo-Anosov element is virtually cyclic. Thus, $N \cap M$ is infinite. 


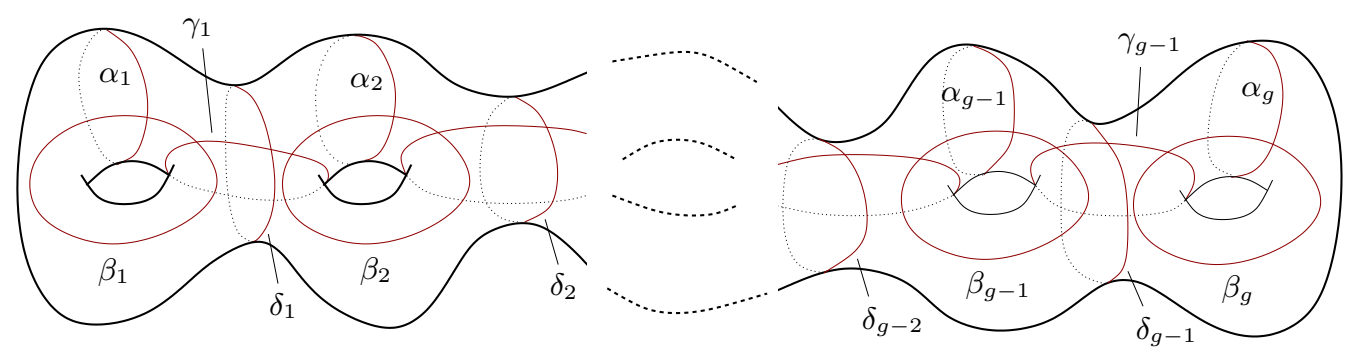

Figure 1. Simple closed loops on the surface of genus $g$.

Proof. The upper bound $\operatorname{ma}(\operatorname{Mod}(g)) \leqslant 6 g-6$ is well known; it comes from the action of $\operatorname{Mod}(g)$ on the character variety parameterizing conjugacy classes of representations of the fundamental group of the surface of genus $g$ in $\mathrm{SL}_{2}$, an affine variety of dimension $6 g-6$. This action is faithful for $g \geqslant 3$ (see [50, Theorem 9.15] and [1, Theorem 4.2]). For $g=2$, the kernel of the action of $\operatorname{Mod}(2)$ on the character variety is the order-2 subgroup generated by the hyperelliptic involution. From [1, Theorem 4.3], or [31, Theorem 6.8], $\operatorname{Mod}(g)$ is virtually torsion-free. Consequently, $\operatorname{Mod}(2)$ contains a finite-index torsion-free subgroup; this subgroup acts faithfully on the character variety.

We now fix a finite-index subgroup $\Gamma$ of $\operatorname{Mod}(g)$, and we assume that $\Gamma$ acts faithfully on a complex quasi-projective variety $X$ of dimension $d$ (note that $X$ is not assumed to be irreducible). Our goal is to obtain the lower bound $d \geqslant 2 g-1$. We identify $\Gamma$ with its image in $\operatorname{Aut}(X)$. Since $\operatorname{Mod}(g)$ is virtually torsion-free, there is a finite-index, torsionfree subgroup in $\Gamma$; we now replace $\Gamma$ by such a group.

The group $\Gamma$ permutes the irreducible components of $X$; let $\Gamma^{\prime}$ be the kernel of this action by permutations. Denote by $X_{i}$ the irreducible components of $X$, and by $\Gamma_{i}^{\prime}$ the kernel of the action of $\Gamma^{\prime}$ on $X_{i}$. The intersection of the $\Gamma_{i}^{\prime}$ is trivial. As $\Gamma$ is torsion-free, either $\Gamma_{i}^{\prime}$ is trivial, or $\Gamma_{i}^{\prime}$ is infinite. Since the intersection of two infinite normal subgroups of $\operatorname{Mod}(g)$ is infinite (Remark 6.4), at least one of the $\Gamma_{i}^{\prime}$ is trivial, and the action of $\Gamma^{\prime}$ on $X_{i}$ is faithful. We replace $\Gamma$ by its finite-index subgroup $\Gamma^{\prime}$ and the variety $X$ by such a component $X_{i}$. Thus, in what follows, $\Gamma$ acts faithfully on the irreducible variety $X$.

We need to show that $d=\operatorname{dim}(X) \geqslant 2 g-1$. Apply Proposition 3.2 to obtain a prime number $p \geqslant 3$ and a good model of the pair $(X, \Gamma)$ over $\mathbf{Z}_{p}$. Then, apply Proposition 3.3. We obtain a finite extension $K$ of $\mathbf{Q}_{p}$, an analytic polydisk $\mathcal{U} \subset X(K)$, and a finite-index subgroup $\Gamma^{\prime \prime}$ of $\Gamma$ that preserves $\mathcal{U}$ : The action of $\Gamma^{\prime \prime}$ on $\mathcal{U}$ is given by an embedding $\Gamma^{\prime \prime}$ into $\operatorname{Diff}^{\text {an }}(\mathcal{U})_{1}$, the group of Tate analytic diffeomorphisms which are equal to Id modulo $p$ (see $\S 2.3 .2$ ). Again, we replace $\Gamma$ by this finite-index subgroup $\Gamma^{\prime \prime}$. Then, the conclusion is a consequence of the following lemma. 
Lemma 6.5. Let $p$ be an odd prime and $K$ be a finite extension of $\mathbf{Q}_{p}$. If a finiteindex subgroup $\Gamma$ of $\operatorname{Mod}(g)$ embeds in $\operatorname{Diff}^{\mathrm{an}}(\mathcal{U})_{1}$, where $\mathcal{U} \simeq \mathcal{O}_{K}^{d}$ is an analytic polydisk of dimension $d$, then $d \geqslant 2 g-1$.

Proof. Elements of $\Gamma$ give Tate analytic diffeomorphisms of $\mathcal{U}$ which are equal to the identity modulo $p$. Since $p \geqslant 3$, we can apply the Bell-Poonen theorem to every element of $\Gamma$ (take $c=1$ in Theorem 2.4). In particular, each element $\gamma \in \Gamma$ determines a Tate analytic vector field $\mathbf{X}_{\gamma}$ on $\mathcal{U}$; and, if $\gamma$ and $\gamma^{\prime}$ commute, then so do the corresponding vector fields (Corollary 2.8).

Denote by $T_{\alpha_{i}}$ and $T_{\beta_{i}}, i=1, \ldots, g$, the Dehn twists along the simple closed loops which are depicted in Figure 1. There exists an integer $m \geqslant 1$ such that the twists $T_{\alpha_{i}}^{m}$ and $T_{\beta_{i}}^{m}$ are all in $\Gamma$. Observe that the $g$ twists $T_{\alpha_{i}}^{m}$ commute. For $x \in \mathcal{U}$, denote by $s(x)$ the dimension of the $K$-vector space spanned by the tangent vectors $\mathbf{X}_{T_{\alpha_{i}}^{m}}(x), 1 \leqslant i \leqslant g$; let $s$ be the maximum of $s(x)$, for $x$ in $\mathcal{U}$.

There exists a smaller polydisk $\mathcal{V} \subset \mathcal{U}$ and a subset $S$ of $\{1, \ldots, g\}$ such that $|S|=s$ and the $\mathbf{X}_{T_{\alpha_{j}}^{m}}(x), j \in S$, are linearly independent at every point $x$ of $\mathcal{V}$. Denote by $\mathbf{X}_{j}$ the vector field $\mathbf{X}_{T_{\alpha_{j}}^{m}}$ for $j$ in $S$. Each $\mathbf{X}_{T_{\alpha_{i}}^{m}}, 1 \leqslant i \leqslant g$, can be written in a unique way as a sum

$$
\mathbf{X}_{T_{\alpha_{i}}^{m}}=\sum_{j \in S} F_{i, j} \mathbf{X}_{j}
$$

where the $F_{i, j}$ 's are analytic functions on $\mathcal{V}$. Since $\left[\mathbf{X}_{T_{\alpha_{l}}^{m}}, \mathbf{X}_{T_{\alpha_{j}}^{m}}\right]=0$ for every pair of indices $l \in\{1, \ldots, g\}$ and $j \in S$, we obtain

$$
\mathbf{X}_{k} F_{i, j}=0
$$

for all $i \in\{1, \ldots, g\}$ and $j, k \in S$.

Suppose that $S \neq\{1, \ldots, g\}$, and pick an index $r$ in $\{1, \ldots, g\} \backslash S$. Observe that $T_{\beta_{r}}^{m}$ does not commute with $T_{\alpha_{r}}^{m}$ but commutes with the other $T_{\alpha_{i}}^{m}$; hence $\left[\mathbf{X}_{T_{\beta_{r}}^{m}}, \mathbf{X}_{j}\right]=0$ for every $j \in S$. Assume by contradiction that, for every $x$ in $\mathcal{V}, \mathbf{X}_{T_{\beta_{r}}^{m}}(x)$ is a linear combination of the $\mathbf{X}_{j}(x), j \in S$, and write $\mathbf{X}_{T_{\beta_{r}}^{m}}=\sum_{j \in S} G_{j} \mathbf{X}_{j}$, where the $G_{j}$ 's are analytic functions on $\mathcal{U}$. The commutation rules imply $\mathbf{X}_{i} G_{j}=0$ for all indices $i$ and $j$ in $S$; thus, equations (6.1) and (6.2) lead to

- $\mathbf{X}_{T_{\alpha_{l}}^{m}} G_{j}=0$ for all indices $l \in\{1, \ldots g\}$;

- $\left[\mathbf{X}_{T_{\alpha_{r}}^{m}}, \mathbf{X}_{T_{\beta_{r}}^{m}}\right]=0$.

Thus, by Corollary 2.8, $T_{\beta_{r}}^{m}$ commutes with $T_{\alpha_{r}}^{m}$, a contradiction. This means that there exists a smaller polydisk $\mathcal{W} \subset \mathcal{V} \subset \mathcal{U}$ on which the vector fields $\left\{\mathbf{X}_{T_{\beta_{r}}^{m}}\right\} \cup\left\{\mathbf{X}_{j}: j \in S\right\}$ are everywhere linearly independent. Now, we add $r$ to $S$ and set $\mathbf{X}_{r}:=\mathbf{X}_{T_{\beta_{r}}^{m}}$.

Use that the $T_{\beta_{i}}^{m}$ commute, and commute with the $T_{\alpha_{j}}^{m}$ for $i \neq j$, and repeat this argument to end up with a set $\mathbf{X}_{i}, i \in S=\{1, \ldots, g\}$, of vector fields which are linearly 
independent on a smaller polydisk; these vector fields correspond to elements of type $T_{\alpha_{j}}^{m}$ or $T_{\beta_{i}}^{m}$, for a disjoint set of curves $\alpha_{j}$ and $\beta_{i}$; we denote by $\Lambda$ this set of curves. Each index $s \in S=\{1, \ldots, g\}$ corresponds to a unique curve $\alpha_{s}$ or $\beta_{s}$ in $\Lambda$; the vector field $\mathbf{X}_{s}$ is determined by $T_{\alpha_{s}}^{m}$ or $T_{\beta_{s}}^{m}$.

In what follows, we fix an element $\Phi$ of the mapping class group which maps this non-separating set of disjoint curves $\Lambda$ to $\alpha_{1}, \alpha_{2}, \ldots, \alpha_{g}$. Then, denote by $\gamma_{i}^{\prime}$ and $\delta_{i}^{\prime}$ the images of the curves $\gamma_{i}$ and $\delta_{i}$, respectively, by $\Phi^{-1}$ (see Figure 1); these curves are disjoint from the $g$ curves of $\Lambda$.

Consider the curves $\gamma_{1}^{\prime}$ and $\delta_{1}^{\prime}$, and fix an integer $m>0$ such that the Dehn twists $T_{\gamma_{1}^{\prime}}^{m}$ and $T_{\delta_{1}^{\prime}}^{m}$ generate a free subgroup of $\Gamma$ (see [31, Theorem 3.14]). These twists commute with the $T_{\nu}^{m}$ for all curves $\nu \in \Lambda$. If, on some polydisk $\mathcal{P} \subset \mathcal{U}$, the vector fields $\mathbf{Y}_{1}$ and $\mathbf{Z}_{1}$ corresponding to $T_{\gamma_{1}^{\prime}}^{m}$ and $T_{\delta_{1}^{\prime}}^{m}$, respectively, are combinations $\mathbf{Y}_{1}=\sum_{j} H_{j} \mathbf{X}_{i}$ and $\mathbf{Z}_{1}=\sum_{j} H_{j}^{\prime} \mathbf{X}_{i}$, then $T_{\gamma_{1}^{\prime}}^{m}$ and $T_{\delta_{1}^{\prime}}^{m}$ commute on that polydisk, and then they commute everywhere, a contradiction. Thus, one can add a vector field $\mathbf{Y}_{1}$ (or $\mathbf{Z}_{1}$ ) to our list of generically independent vector fields. Playing the same game with the curves $\gamma_{k}^{\prime}$ and $\delta_{k}^{\prime}$ for $2 \leqslant k \leqslant g-1$, we end up with $2 g-1$ vectors fields, and deduce that $\operatorname{dim}(\mathcal{U}) \geqslant 2 g-1$.

The group $\operatorname{Out}\left(\mathbb{F}_{2 n+s-1}\right)$ contains a copy of $\operatorname{Mod}(n, s)$, the mapping class group of the surface of genus $n$ with $s \geqslant 1$ punctures. The proof of Theorem D also shows that

$$
2 n-1 \leqslant \operatorname{ma}(\operatorname{Mod}(n, 1)) \leqslant \operatorname{ma}\left(\operatorname{Out}\left(\mathbb{F}_{2 n}\right)\right)
$$

(with $s=1$ ) and that

$$
2 n-1 \leqslant \operatorname{ma}(\operatorname{Mod}(n, 2)) \leqslant \operatorname{ma}\left(\operatorname{Out}\left(\mathbb{F}_{2 n+1}\right)\right)
$$

(with $s=2$ ). Thus, we obtain $m-2 \leqslant \operatorname{ma}\left(\operatorname{Out}\left(\mathbb{F}_{m}\right)\right.$ ) for all $m \geqslant 2$.

\subsection{Nilpotent groups}

Let $H$ be a group. Define $H^{(1)}=[H, H]$, the derived subgroup of $H$, generated by all commutators $a b a^{-1} b^{-1}$ with $a$ and $b$ in $H$, and then inductively $H^{(r)}=\left[H^{(r-1)}, H^{(r-1)}\right]$. The first integer $r \geqslant 1$ such that $H^{(r)}$ is trivial is called the derived length of $H$; such an $r$ exists if and only if $H$ is solvable. This integer is denoted by $\mathrm{dl}(H)$, and a similar notation is used for Lie algebras. Then, define the virtual derived length of $H$ by

$$
\operatorname{vdl}(H)=\min \left\{\operatorname{dl}\left(H^{\prime}\right): H^{\prime} \text { is a finite-index subgroup of } H\right\} .
$$

THEOREM 6.6. Let $H$ be a finitely generated nilpotent group. If $H$ acts faithfully by automorphisms on an irreducible complex quasi-projective variety $X$, then we have $\operatorname{vdl}(H) \leqslant \operatorname{dim}(X)$. Thus, $\operatorname{ma}(H) \geqslant \operatorname{vdl}(H)$. 
Let us sketch the proof. Let $d$ be the dimension of $X$. Apply Propositions 3.2 and 3.3: there is a finite-index subgroup $H_{0}$ of $H$ and a polydisk $\mathcal{U} \simeq \mathbf{Z}_{p}{ }^{d} \subset X\left(\mathbf{Z}_{p}\right)$ such that the action of $H_{0}$ on $X\left(\mathbf{Z}_{p}\right)$ preserves the polydisk $\mathcal{U}$ and determines an embedding of $H_{0}$ in $\operatorname{Diff}^{\text {an }}(\mathcal{U})_{1}$. Consider the Lie algebra $\mathfrak{h}$ which is generated by the vector fields $X_{f}$, for $f$ in $H_{0} \subset \operatorname{Diff}^{\text {an }}(\mathcal{U})_{1}$. Corollary 2.8 shows that the derived length of $\mathfrak{h}$ is equal to the derived length of $H_{0}$. To conclude, apply the following lemma, the proof of which is the same as in [15, Proposition 3.9], or [29, Theorem 1.1].

Lemma 6.7. Let $\mathfrak{h}$ be a nilpotent Lie algebra of Tate analytic vector fields on a polydisk $\mathcal{U}$. Then, $\operatorname{dl}(\mathfrak{h}) \leqslant \operatorname{dim}(\mathcal{U})$.

\section{Periodic orbits and invariant polydisks}

Our goal is to produce invariant $p$-adic polydisks for some groups of birational transformations of a projective variety defined over a finite extension $K$ of $\mathbf{Q}_{p}$. This is closely related to the existence of "good" periodic orbits for groups of birational transformations defined over finite fields; in a first time, we focus on the construction of such orbits.

\subsection{Property $\left(\tau^{\infty}\right)$ and linear isoperimetric inequalities}

In this section, we introduce property $\left(\tau^{\infty}\right)$, which may be viewed as a weak form of Kazhdan property (T) (see [40]), and we relate this property to linear isoperimetric inequalities. Then, we prove that property $\left(\tau^{\infty}\right)$ is equivalent to property $(\mathrm{FM})$, introduced in [35] and [22].

In what follows, $\Gamma$ will be a group with a finite symmetric set of generators $S$ (the symmetry means that $s \in S$ if and only if $s^{-1} \in S$ ). If $h$ is an element of $\Gamma,|h|_{S}$ denotes the length of $h$ with respect to $S$; by definition, $|h|_{S}$ is the minimum of the integers $m \geqslant 0$ such that $h$ is a product of $m$ elements of $S$.

\subsubsection{Quotients and Schreier graphs}

Given a subgroup $R$ of $\Gamma$, consider the quotient space $\Gamma / R$. The group $\Gamma$ acts on $\Gamma / R$ by left translations: given $h$ in $\Gamma$, we denote by $L_{h}$ the translation $g R \mapsto h g R$. Denote by $\ell^{2}(\Gamma / R)$ the space of $\ell^{2}$-functions on $\Gamma / R$, i.e. functions $\varphi: \Gamma / R \rightarrow \mathbf{C}$ which are square integrable:

$$
\|\varphi\|_{\ell^{2}(\Gamma / R)}^{2}:=\sum_{\omega \in \Gamma / R}|\varphi(\omega)|^{2}<\infty
$$


The action of $\Gamma$ on $\Gamma / R$ by left translations determines a unitary representation $g \mapsto L_{g^{-1}}^{*}$ of $\Gamma$ on $\ell^{2}(\Gamma / R)$, where $L_{g^{-1}}^{*} \varphi:=\varphi \circ L_{g^{-1}}$.

The Schreier graph $\mathcal{G}_{R}$ is defined as follows: The set of vertices of $\mathcal{G}_{R}$ is $\mathcal{G}_{R}^{0}=\Gamma / R$; two vertices $g_{1} R$ and $g_{2} R \in \Gamma / R$ are joined by an edge if and only if there exists $s \in S$ satisfying $g_{2} R=s g_{1} R$. When $R=\{e\}, \mathcal{G}_{R}$ is the Cayley graph $\mathcal{G}:=\mathcal{G}_{\{e\}}$ of $\Gamma$. Those graphs depend on the choice of the generating set $S$.

Remark 7.1. If the distance between $g R$ and $g^{\prime} R$ in the graph $\mathcal{G}_{R}$ is $\delta$, then

$$
\operatorname{dist}\left(L_{h}(g R), L_{h}\left(g^{\prime} R\right)\right)
$$

is at most $\delta+2|h|_{S}$. When $R$ is a normal subgroup of $\Gamma$, then $\Gamma$ also acts on the right, $g R \mapsto g R h=g h R$, and this right action is by isometries.

Let $\Omega$ be a finite subset of $\Gamma / R$. Denote by $\chi_{\Omega}: \Gamma / R \rightarrow\{0,1\}$ the characteristic function of $\Omega$, i.e. $\chi_{\Omega}(x)=1$ if and only if $x \in \Omega$. Since $\Omega$ is finite, $\chi_{\Omega}$ is square integrable. An element $x \in \Omega$ is in the boundary $\partial \Omega$ of $\Omega$ if and only if there exists an element $y$ of $(\Gamma / R) \backslash \Omega$ which is connected to $x$ by an edge of $\mathcal{G}_{R}$; in other words, $x \in \partial \Omega$ if and only if $x \in \Omega$ and there exists $s \in S$ such that $L_{s}(x) \notin \Omega$, that is, if and only if $\chi_{\Omega}(x)=1$ and there exists $s \in S$ such that $\left(L_{s}^{*} \chi_{\Omega}\right)(x)=0$. Thus, we have

$$
\left\|\chi_{\Omega}-L_{s}^{*}\left(\chi_{\Omega}\right)\right\|_{\ell^{2}(\Gamma / R)}^{2}=\sum_{x \in \Gamma / R}\left(\chi_{\Omega}(x)-\chi_{\Omega}\left(L_{s} x\right)\right)^{2} \leqslant \sum_{x \in \bigcup_{s \in S} \Omega \triangle s^{-1}(\Omega)} 1^{2},
$$

and hence

$$
\left\|\chi_{\Omega}-L_{s}^{*}\left(\chi_{\Omega}\right)\right\|_{\ell^{2}(\Gamma / R)}^{2} \leqslant 2|S||\partial \Omega| .
$$

The Cheeger constant of the Schreier graph $\mathcal{G}_{R}$ is the infimum

$$
h\left(\mathcal{G}_{R}\right)=\inf _{\Omega} \frac{|\partial \Omega|}{|\Omega|}
$$

where $\Omega$ describes the non-empty finite subsets of $\Gamma / R$ with $|\Omega| \leqslant \frac{1}{2}|\Gamma / R|$ (this constraint is void when $\Gamma / R$ is infinite).

\subsubsection{Uniform, linear isoperimetric inequalities and property $\left(\tau^{\infty}\right)$}

Let $\Gamma$ be a group with a finite, symmetric set of generators $S$. The group $\Gamma$ has property $\left(\tau^{\infty}\right)$ if there exists a constant $\varepsilon=\varepsilon(\Gamma, S)>0$ such that, for every subgroup $R \subset \Gamma$ of infinite index and every function $\xi \in \ell^{2}(\Gamma / R)$, there exists an element $s \in S$ such that $\left\|\xi-L_{s}^{*} \xi\right\| \geqslant \varepsilon\|\xi\|$. Property $\left(\tau^{\infty}\right)$ does not depend on $S$, even if the constant $\varepsilon$ does (this follows from Proposition 7.3 below); thus, we refer to property $\left(\tau^{\infty}\right)$ as a property of the (finitely generated) group $\Gamma$, and not of the pair $(\Gamma, S)$. 
Proposition 7.2. Let $\Gamma$ be a group with a finite, symmetric set of generators $S$. Then, $\Gamma$ has property $\left(\tau^{\infty}\right)$ if and only if there is a positive constant $h_{\infty}$ such that $h\left(\mathcal{G}_{R}\right) \geqslant h_{\infty}>0$ for every subgroup $R$ of $\Gamma$ of infinite index.

In fact, $h\left(\mathcal{G}_{R}\right) \geqslant \varepsilon^{2} / 2|S|$ if $\Gamma$ satisfies property $\left(\tau^{\infty}\right)$ with constant $\varepsilon$ for $S$.

Proof. Assume that $\Gamma / R$ is infinite. Consider the unitary action of $\Gamma$ on $\ell^{2}(\Gamma / R)$ by left translations. For every finite set $\Omega \subseteq \Gamma / R$, the characteristic function $\chi_{\Omega}$ is an element of $\ell^{2}(\Gamma / R)$ and property $\left(\tau^{\infty}\right)$ implies the existence of an element $s \in S$ such that

$$
\left\|\chi_{\Omega}-L_{s}^{*} \chi_{\Omega}\right\|_{\ell^{2}(\Gamma / R)} \geqslant \varepsilon\left\|\chi_{\Omega}\right\|_{\ell^{2}(\Gamma / R)}=\varepsilon|\Omega|^{1 / 2} .
$$

From inequality (7.1), we deduce that $(2|S||\partial \Omega|)^{1 / 2} \geqslant \varepsilon|\Omega|^{1 / 2}$. Hence, $h\left(\mathcal{G}_{R}\right) \geqslant h_{\infty}$ for $h_{\infty}=\varepsilon^{2} / 2|S|$.

The other implication may be obtained as in Hulanicki's characterization of amenability (see [49, Theorems 3.1.5 and 4.3.2]). We do not prove it because it is not used in this article.

\subsubsection{Other classical properties and examples}

We now compare property $\left(\tau^{\infty}\right)$ to other classical properties.

- A group $\Gamma$ has property $(\tau)$ if there exists a constant $\varepsilon>0$ such that, for every finiteindex normal subgroup $R \subset \Gamma$, and for every function $\xi: \Gamma / R \rightarrow \mathbf{C}$ which is $\ell^{2}$-orthogonal to the constant functions, there exists a generator $s \in S$ such that $\left\|\xi-L_{s}^{*} \xi\right\| \geqslant \varepsilon\|\xi\|$ (see [49]). In [5], Bekka and Olivier study property $\left(\mathrm{T}_{\ell^{p}}\right)$, for $p \neq 2$, and show that this property is equivalent to the conjunction of properties $(\tau)$ and $\left(\tau^{\infty}\right)$. In particular, property $\left(\mathrm{T}_{\ell^{p}}\right)$ implies property $\left(\tau^{\infty}\right)$.

- In [22], Cornulier introduces property (FM). Let us describe this property in the case of a discrete group $\Gamma$. A discrete $\Gamma$-set is, by definition, an action of $\Gamma$ on a discrete set $X$. A mean on $X$ (or more precisely on $\ell^{\infty}(X)$ ), is a linear functional $m: \ell^{\infty}(X) \rightarrow \mathbf{R}$ that satisfies $m(\mathbb{1})=1$ and $m(\xi) \geqslant 0$ for every bounded function $\xi: X \rightarrow \mathbf{R}_{+}$. A mean is $\Gamma$-invariant if its values on $\xi$ and $\xi \circ \gamma^{-1}$ are equal for all $\xi \in \ell^{\infty}(X)$ and $\gamma \in \Gamma$. One says that $\Gamma$ has property $(\mathrm{FM})$ if every discrete $\Gamma$-set with a $\Gamma$-invariant mean contains a finite $\Gamma$-orbit. From [22], we get the following result.

Proposition 7.3. Discrete groups with property (FM) are finitely generated. Property $(\mathrm{FM})$ is equivalent to property $\left(\tau^{\infty}\right)$.

Thus, we could have started with property (FM), without assuming $\Gamma$ to be finitely generated, and then deduce property $\left(\tau^{\infty}\right)$, which is really the definition that we use in the sequel. 
Proof. The first assertion is contained in [22, Proposition 5.6]. [22, Remark 5.16] shows that property $(\mathrm{FM})$ implies property $\left(\tau^{\infty}\right)$. The argument is the following. Assume that there is a sequence of infinite quotient spaces $Y_{n}=\Gamma / R_{n}$ and functions $\xi_{n} \in \ell^{2}\left(Y_{n}\right)$ of norm 1 such that $\left\|\xi_{n}-L_{s}^{*} \xi_{n}\right\| \leqslant 1 / n$. Consider the discrete $\Gamma$-set $X$ which is obtained as the disjoint union of the $Y_{n}$, and extend each $\xi_{n}$ as a function on $X$ by $\xi_{n}(y)=0$ if $y \in X \backslash Y_{n}$. The linear maps $m_{n}(\xi)=\sum_{x} \xi_{n}(x)^{2} \xi(x)$ define a sequence of means on $X$. By compactness of the set of means, a subsequence $\left\{m_{n_{i}}\right\}_{i}$ converges towards a mean $m_{\infty}$; by construction, $m_{\infty}$ is $\Gamma$-invariant. But $X$ does not contain any finite orbit. Thus, (FM) implies $\left(\tau^{\infty}\right)$.

In the opposite direction, assume that $\Gamma$ has property $\left(\tau^{\infty}\right)$ but does not have property $(\mathrm{FM})$. Then, there exists a discrete $\Gamma$-set $X$, which is a disjoint union of infinite orbits $Y_{i}=\Gamma / R_{i}$ and which supports an invariant mean $m$. The existence of $m$ implies that $\ell^{2}(X)$ contains a sequence of almost invariant vectors $\xi_{n}$ (see [22, Lemma 5.9] and [4, Appendix G.3]): $\xi_{n}$ has norm 1 and $\left\|\xi_{n}-L_{s}^{*} \xi_{n}\right\| \leqslant 1 / n$ for all $s$ in the generating set $S$. To obtain a contradiction, decompose $\xi_{n}$ as an orthogonal sum $\xi_{n}=\sum_{i} \xi_{n, i}$, where each $\xi_{n, i}$ is the restriction of $\xi_{n}$ to the orbit $Y_{i}$. Since $\Gamma / R_{i}$ is infinite, there exists a generator $s_{n, i}$ such that $\left\|\xi_{n, i}-L_{s_{n, i}}^{*} \xi_{n, i}\right\|^{2} \geqslant \varepsilon^{2}$ (with $\varepsilon$ the constant provided by property $\left(\tau^{\infty}\right)$ ). Hence, there is a generator $s_{n}$ such that $\left\|\xi_{n}-L_{s_{n}}^{*} \xi_{n}\right\|^{2} \geqslant \varepsilon^{2} /|S|$. We get a contradiction when $n^{2}>|S| / \varepsilon^{2}$.

- A discrete group $\Gamma$ has Kazhdan property $(\mathrm{T})$ if every action of $\Gamma$ on a Hilbert space $\mathcal{H}$ by affine isometries has a fixed point (see [40, Chapters 1 and 4]). Such a group is automatically finitely generated and, given a finite system of generators, the following equivalent definition of Kazhdan property $(\mathrm{T})$ will be more useful to us. A finitely generated group $\Gamma$ has Kazhdan property $(\mathrm{T})$ if, for any finite symmetric set of generators $S$, there exists an $\varepsilon>0$, with the following property: given any unitary representation $\varrho$ of $\Gamma$ on a Hilbert space $\mathcal{H}$, either there exists $v \in \mathcal{H} \backslash\{0\}$ such that $\varrho(\Gamma) \cdot v=v$, or, for every $v \in \mathcal{H}$, there exists $s \in S$ such that

$$
\|v-\varrho(s) \cdot v\| \geqslant \varepsilon\|v\|
$$

Such a positive number $\varepsilon$ is called a Kazhdan constant for the pair $(\Gamma, S)$. Thus, property $\left(\tau^{\infty}\right)$ turns out to be a weak form of property $(\mathrm{T})$, in which one only considers the unitary representations $\ell^{2}(Y)$, where $Y$ is a set on which $\Gamma$ acts transitively. We obtain the following result.

Proposition 7.4. If $\Gamma$ satisfies Kazhdan property $(\mathrm{T})$ with constant $\varepsilon$ for the generating set $S$, then it satisfies the uniform linear isoperimetric inequality $|\partial \Omega| \geqslant\left(\varepsilon^{2} / 2|S|\right)|\Omega|$ in all its infinite quotients $\Gamma / R$. 
Example 7.5. Let $\Gamma$ be a non-amenable group with no infinite proper subgroup. Then $\Gamma$ has property $\left(\tau^{\infty}\right)$ (see [5, Proposition 15 and Example 17], or [35, §4.C]). Let $\Gamma$ be an irreducible lattice in $\mathrm{SO}(n, 2) \times \mathrm{SO}(n+1,1)$, for some $n \geqslant 3$. Then $\Gamma$ does not have property $(\mathrm{T})$, but has property $\left(\tau^{\infty}\right)$ (see [5, Example 14] and [22, Example 1.14] for a related construction).

\subsection{Finite orbits and finite-index subgroups}

Let $X$ be an absolutely irreducible projective variety of dimension $d$ defined over a finite field $\mathbf{F}$. Let $\Gamma$ be a group with a finite symmetric set of generators $S$. Assume that the group $\Gamma$ embeds into the group $\operatorname{Bir}\left(X_{\mathbf{F}}\right)$ and identify $\Gamma$ with its image $\operatorname{in} \operatorname{Bir}\left(X_{\mathbf{F}}\right)$.

\subsubsection{The escaping set $E$}

Let $U$ be a Zariski open subset of $X$ defined over $\mathbf{F}$ such that, for every $s \in S$, the map $\left.s\right|_{U}: U \rightarrow X$ is a morphism and an open immersion. Such a set exists because $S$ is finite: for $U$, take the complement of all the proper subsets $B_{s}$, for $s$ in $S$, where $B_{s}$ is defined as in $§ 3.1 .2$.

Remark 7.6. One may want to shrink $U$ in certain situations. For instance, given an element $f$ of the group $\Gamma$, with $f \neq \mathrm{Id}$, one may remove the set of fixed points of $f$ from $X$, and take $U \subset X \backslash\{x: f(x)=x\}$. Or one can remove the singular locus of $X$ from $U$.

By construction, the codimension of the Zariski closed set $X \backslash U$ is at least 1 . Let $E \subseteq U$ be the subset of points that may escape $U$ when one applies one of the generators:

$$
E:=\bigcup_{s \in S} s^{-1}(X \backslash U)
$$

where $s^{-1}(X \backslash U)$ is the total transform of the Zariski closed set $X \backslash U$. This escaping set $E$ is a proper, Zariski-closed subset of $U$.

\subsubsection{Lang-Weil estimates (see [46])}

By Lang-Weil estimates, there exists a positive constant $c_{U}$ such that, given any finite field extension $\mathbf{F}^{\prime}$ of $\mathbf{F}$, the number of points in $U\left(\mathbf{F}^{\prime}\right)$ satisfies

$$
\left|\mathbf{F}^{\prime}\right|^{d}-c_{U}\left|\mathbf{F}^{\prime}\right|^{d-1 / 2} \leqslant\left|U\left(\mathbf{F}^{\prime}\right)\right| \leqslant\left|\mathbf{F}^{\prime}\right|^{d}+c_{U}\left|\mathbf{F}^{\prime}\right|^{d-1 / 2},
$$

where $d=\operatorname{dim} U=\operatorname{dim} X$ (the constant $c_{U}$ does not depend on $\mathbf{F}^{\prime}$ ). 
Similarly,

$$
\left|E\left(\mathbf{F}^{\prime}\right)\right| \leqslant b_{E}\left|\mathbf{F}^{\prime}\right|^{d-1}+c_{E}\left|\mathbf{F}^{\prime}\right|^{d-3 / 2},
$$

where $b_{E}$ is the number of absolutely irreducible $(d-1)$-dimensional components of $E$; the constants $b_{E}$ and $c_{E}$ depend on $E$ but not on $\mathbf{F}^{\prime}$.

\subsubsection{Regular stabilizers}

Fix a finite extension $\mathbf{F}^{\prime}$ of the field $\mathbf{F}$. Given a point $x \in U\left(\mathbf{F}^{\prime}\right)$, one associates a subgroup $R_{x}$ of $\Gamma$ which will be called the regular stabilizer of $x$. To define it, we proceed as follows. Let $\left(e, g_{1}, \ldots, g_{l}\right)$ be a path in the Cayley graph $\mathcal{G}$, and denote by $s_{i+1}$ the element of $S$ such that $g_{i+1}=s_{i+1} g_{i}, 1 \leqslant i \leqslant l-1$. The path $\left(e, g_{1}, \ldots, g_{l}\right)$ is a regular path if

(i) $s_{1}$ is well defined at $x_{0}:=x$ and maps $x_{0}$ to a point $x_{1} \in U$;

(ii) for all $i \leqslant l-1, s_{i+1}$ maps $x_{i}$ to a point $x_{i+1} \in U$ (since $x_{i}$ is in $U, s_{i+1}$ is well defined at $x_{i}$ ).

Thus, the notion of regular path depends on the starting point $x$. By definition, the regular orbit of $x$ is the set of all points $g_{l}(x)$ for all regular paths $\left(e, g_{1}, \ldots, g_{l}\right)$. The regular orbit of $x$ may intersect the escaping set $E$; when it does, we simply do not apply an element of $S$ that would make it leave $U$.

Definition 7.7. An element $g \in \Gamma$ is a regular stabilizer of $x \in U\left(\mathbf{F}^{\prime}\right)$ if there exists a regular path $\left(e, g_{1}, \ldots, g_{l}\right)$ in $\mathcal{G}$ such that (i) $g_{l}=g$ and (ii) $g_{l}(x)=x$. The set of all regular stabilizers is the regular stabilizer of $x$, and is denoted by $R_{x}$.

LEMMA 7.8. The regular stabilizer $R_{x}$ is a subgroup of $\Gamma$.

Proof. Given $g$ and $h$ in $R_{x}$, and regular paths $\left(e, g_{1}, \ldots, g_{l}\right)$ and $\left(e, h_{1}, \ldots, h_{l^{\prime}}\right)$ in $\Gamma$ satisfying properties (i) and (ii) of Definition 7.7 for $g$ and $h$, respectively, one can define a new regular path $\left(e, h_{1}, \ldots, h_{l^{\prime}}, g_{1} h_{l^{\prime}}, \ldots, g_{l} h_{l^{\prime}}\right)$ which fixes $x$; thus, $g \circ h$ is an element of $R_{x}$. Similarly, write $g_{i+1}=s_{i+1} g_{i}, s_{i+1} \in S, x_{0}=x$, and $x_{i+1}=s_{i+1}\left(x_{i}\right)$ for $0 \leqslant i \leqslant l-1$. By construction of $U$ and symmetry of $S, s_{i+1}$ is a regular automorphism from a neighborhood of $x_{i}$ to a neighborhood of $x_{i+1}$; hence, $s_{i+1}^{-1}$ is well defined at $x_{i+1}$. One can therefore reverse the regular path and get a path $\left(e, s_{l}^{-1}, s_{l-1}^{-1} \circ s_{l}^{-1}, \ldots, g^{-1}\right)$ which starts at $x_{l}$ and ends at $x_{0}$. In our case, $x_{l}=x=x_{0}$, and we conclude that $g^{-1}$ is an element of $R_{x}$.

This proof shows that we can concatenate and reverse regular paths. The evaluation map $\mathrm{ev}_{x}$ takes a regular path $\left(e, g_{1}, \ldots, g_{l}\right)$ and gives a point

$$
\mathrm{ev}_{x}\left(e, g_{1}, \ldots, g_{l}\right)=g_{l}(x)
$$


We shall say that an element $g \in \Gamma$ is very well defined at $x \in U\left(\mathbf{F}^{\prime}\right)$ if there is a regular path from $e$ to $g_{l}=g$. For such an element, the image $\mathrm{ev}_{x}\left(e, g_{1}, \ldots, g_{l}\right)=g_{l}(x)=g(x)$ does not depend on the choice of the regular path joining $e$ to $g$. As a consequence, the evaluation map is defined on the set of elements of $\Gamma$ which are very well defined at $x$, and maps it into the set $U\left(\mathbf{F}^{\prime}\right)$. The preimage of $x$ is the regular stabilizer. The image is the regular orbit of $x$.

\subsubsection{The subsets $\Omega_{x} \subseteq \Gamma / R_{x}$}

Fix a point $x \in U\left(\mathbf{F}^{\prime}\right)$. Given an element $g \in \Gamma$ which is very well defined at $x$, one gets a point $g(x) \in U$, as well as a vertex $[g]:=g R_{x}$ in the graph of cosets $\mathcal{G}_{R_{x}}$ for the regular stabilizer $R_{x}$ of $x$. We define $\Omega_{x} \subseteq \Gamma / R_{x}$ to be the set of all such vertices $[g]$. The evaluation map determines a map ev $\mathrm{ev}_{x} \Omega_{x} \rightarrow U\left(\mathbf{F}^{\prime}\right)$ (we make use of the same notation for simplicity).

Proposition 7.9. Let $\mathbf{F}^{\prime}$ be a finite extension of $\mathbf{F}$. Let $x$ be a point of $U\left(\mathbf{F}^{\prime}\right)$. The subset $\Omega_{x} \subseteq \Gamma / R_{x}$ satisfies the following properties:

(1) $\Omega_{x}$ contains $[e]$;

(2) $\Omega_{x}$ is connected: for every $[g] \in \Omega_{x}$ there is a path in $\mathcal{G}_{R_{x}}$, corresponding to a regular path $\left(e, g_{1}, \ldots, g_{l}\right)$ in $\Gamma$, which connects $[e]$ to $[g]$ in $\Omega_{x}$;

(3) the evaluation map $\mathrm{ev}_{x}:[g] \mapsto g(x)$ is well defined (because $R_{x}$ stabilizes $x$ ) and is an injective map $\mathrm{ev}_{x}: \Omega_{x} \rightarrow U\left(\mathbf{F}^{\prime}\right)$, the image of which is the regular orbit of $x$;

(4) $\Omega_{x}$ is a finite set, with $\left|\Omega_{x}\right| \leqslant\left|U\left(\mathbf{F}^{\prime}\right)\right|$.

Proof. All we have to prove is that $\mathrm{ev}_{x}$ is injective. If $g(x)=h(x)$ with two regular paths $\left(e, g_{1}, \ldots, g_{l}=g\right)$ and $\left(e, h_{1}, \ldots, h_{l^{\prime}}=h\right)$, one can reverse the path from $e$ to $h_{l^{\prime}}=h$ and get a regular path that maps $x$ to $h^{-1} \circ g(x)=x$; this means that $h^{-1} \circ g \in R_{x}$.

Thus, one gets a parametrization of the regular orbit of $x \in U\left(\mathbf{F}^{\prime}\right)$ by the set $\Omega_{x}$. An element $[g] \in \Omega_{x}$ is a boundary point of $\Omega_{x}$ in the graph $\mathcal{G}_{R_{x}}$ if and only if there is a generator $s \in S$ such that $[s g] \notin \Omega_{x}$; this means that $s$ is not a regular automorphism from a neighborhood of $g(x)$ to its image $s(x): g(x)$ escapes from $U$ when one applies $s$, and therefore $g(x) \in E\left(\mathbf{F}^{\prime}\right)$. Since the evaluation map is injective, one gets

$$
\left|\partial \Omega_{x}\right|=\left|\mathrm{ev}_{x}\left(\partial \Omega_{x}\right)\right|=\left|E_{x}\left(\mathbf{F}^{\prime}\right)\right|
$$

where $E_{x}\left(\mathbf{F}^{\prime}\right)$ is the subset of $E\left(\mathbf{F}^{\prime}\right)$ which is equal to $\mathrm{ev}_{x}\left(\partial \Omega_{x}\right)$.

Since regular orbits are disjoint, the sets $E_{x}\left(\mathbf{F}^{\prime}\right)$ and $E_{y}\left(\mathbf{F}^{\prime}\right)$ are disjoint as soon as $x$ and $y$ are not in the same regular orbit. Being finite, $U\left(\mathbf{F}^{\prime}\right)$ is a union of finitely many 
disjoint regular orbits. Fixing a set $\left\{x_{1}, \ldots, x_{m}\right\}$ of representatives of these regular orbits, we obtain

$$
U\left(\mathbf{F}^{\prime}\right)=\bigsqcup_{i=1}^{m} \operatorname{ev}_{x_{i}}\left(\Omega_{x_{i}}\right) .
$$

Let us now assume that $\Gamma$ has property $\left(\tau^{\infty}\right)$; thus, by Proposition $7.2, \Gamma$ satisfies a uniform linear isoperimetric inequality

$$
|\partial \Omega| \geqslant \frac{\varepsilon^{2}}{2|S|}|\Omega|
$$

in all its infinite Schreier graphs $\mathcal{G}_{R}$. Suppose that $R_{x}$ has infinite index in $\Gamma$ for every $x \in U\left(\mathbf{F}^{\prime}\right)$. Then,

$$
\begin{aligned}
\left|U\left(\mathbf{F}^{\prime}\right)\right| & =\sum_{i=1}^{m}\left|\mathrm{ev}_{x_{i}}\left(\Omega_{x_{i}}\right)\right|=\sum_{i=1}^{m}\left|\Omega_{x_{i}}\right| \\
& \leqslant \sum_{i=1}^{m} \frac{2|S|}{\varepsilon^{2}}\left|\partial \Omega_{x_{i}}\right|=\sum_{i=1}^{m} \frac{2|S|}{\varepsilon^{2}}\left|E_{x_{i}}\left(\mathbf{F}^{\prime}\right)\right| \leqslant \frac{2|S|}{\varepsilon^{2}}\left|E\left(\mathbf{F}^{\prime}\right)\right| .
\end{aligned}
$$

Then, the Lang-Weil estimates stated in equations (7.2) and (7.3) imply that

$$
\left|\mathbf{F}^{\prime}\right|^{d} \leqslant c_{U}\left|\mathbf{F}^{\prime}\right|^{d-1 / 2}+\frac{2|S|}{\varepsilon^{2}}\left(b_{E}\left|\mathbf{F}^{\prime}\right|^{d-1}+c_{E}\left|\mathbf{F}^{\prime}\right|^{d-3 / 2}\right) .
$$

Thus, if the degree of the extension is large enough (e.g. if $\left.\left|\mathbf{F}^{\prime}\right|^{1 / 2} \geqslant c_{U}+2|S|\left(b_{E}+c_{E}\right) / \varepsilon^{2}\right)$, one gets a contradiction. This provides a proof of the following theorem.

THEOREM 7.10. Let $X$ be an absolutely irreducible projective variety defined over a finite field $\mathbf{F}$. Let $\Gamma$ be a subgroup of $\operatorname{Bir}\left(X_{\mathbf{F}}\right)$ with property $\left(\tau^{\infty}\right)$ and $S$ be a finite symmetric set of generators of $\Gamma$. Let $U$ be a non-trivial, Zariski open subset of $X$ such that, for every $s \in S$, the map $\left.s\right|_{U}: U \rightarrow X$ is an open immersion. If $\mathbf{F}^{\prime}$ is a finite extension of $\mathbf{F}$ and $\left|\mathbf{F}^{\prime}\right|$ is large enough, there exists a point $x$ in $U\left(\mathbf{F}^{\prime}\right)$ such that the regular stabilizer $R_{x}$ of $x$ is a finite-index subgroup of $\Gamma$.

\subsubsection{Abelian groups}

Let $\alpha \geqslant 1$ be a real number. Say that a graph $\mathcal{G}$ satisfies an isoperimetric inequality of type $\alpha$ if there is a constant $c>0$ such that

$$
|\partial \Omega|^{\alpha} \geqslant c|\Omega|
$$

for every finite subset $\Omega$ of $\mathcal{G}$. Let $d \geqslant 2$ be an integer. The Cayley graph of the group $\mathbf{Z}^{d}$ satisfies an isoperimetric inequality of type $d /(d-1)$ for any finite symmetric set of 
generators; the isoperimetric inequality satisfied in Proposition 7.2 is of linear type (i.e. $\alpha=1)$. If $\mathcal{G}$ satisfies an isoperimetric inequality of type $\alpha$, for some constant $c>0$, it satisfies the isoperimetric inequality of type $\beta$ for every $\beta \geqslant \alpha$ with the same constant $c$.

Given a group $\Gamma$, with a finite symmetric set of generators $S$, denote by $B(r)$ the ball of radius $r$ in the Cayley graph $\mathcal{G}=\mathcal{G}(\Gamma, S)$. The number of vertices in $B(r)$ is denoted by $|B(r)|$. Then, define the function $\Phi_{S}$ by

$$
\Phi_{S}(t)=\min \{r: t \leqslant|B(r)|\}
$$

(as in $[24, \S 1$, p. 295]). For instance, if $\Gamma$ is a free abelian group of rank $d$, and $S$ is any finite symmetric set of generators, one can find a subset $S^{\prime}$ of $S$ such that $S^{\prime}$ forms a basis of the vector space $\Gamma \otimes_{\mathbf{z}} \mathbf{Q}$. The set $S^{\prime}$ has $d$ elements; thus, the ball of radius $r$ in $\mathcal{G}(\Gamma, S)$ contains at least $(1+2 r)^{d}$ elements. This implies that $\Phi_{S}(t) \leqslant t^{1 / d}$. Coulhon and Saloff-Coste proved in [24] that

$$
\frac{|\partial \Omega|}{|\Omega|} \geqslant \frac{1}{8|S| \Phi_{S}(2|\Omega|)}
$$

for every non-empty finite subset of a group $\Gamma$. We shall use this inequality to give a short proof of the following lemma, which provides a uniform constant $c_{S}$ for the isoperimetric inequality in quotients of abelian groups.

Lemma 7.11. Let $A$ be a free abelian group of rank $k>1$, and let $S$ be a finite symmetric set of generators of $A$. Fix an integer $l<k$; set $q=k-l$ and $c_{S}=(16|S|)^{-(q-1) / q}$. Then, given any subgroup $R$ of $A$ of rank at most $l$, and any finite subset $\Omega$ of the Cayley graph $\mathcal{G}(A / R, S)$, we have

$$
|\partial \Omega|^{q / q-1} \geqslant c_{S}|\Omega|
$$

Proof. The group $R$ is contained in a subgroup $T$ of $\mathrm{A}$ such that $A / T$ is a free abelian group of rank at least $q$. In the group $A / T$, with the set of generators given by the projection of $S$, the function $\Phi_{S}$ satisfies $\Phi_{S}(t) \leqslant t^{1 / q}$. The projection $A / R \rightarrow A / T$ maps the ball of radius $r$ in the Cayley graph $\mathcal{G}(A / R, S)$ onto the ball of the same radius in $\mathcal{G}(A / T, S)$. Thus, the function $\Phi_{S}$ for $A / R$ satisfies the same inequality $\Phi_{S}(t) \leqslant t^{1 / q}$. This implies that

$$
|\partial \Omega| \geqslant(8|S|)^{-1} 2^{-1 / q}|\Omega|^{(q-1) / q},
$$

and the result follows.

TheOREM 7.12. Let $X$ be a projective variety, defined over a finite field $\mathbf{F}$, and let $d$ be its dimension. Let $A$ be a free abelian group of rank $k<\infty$, acting by birational transformations on $X$ (defined over $\mathbf{F}$ ). Then, there exist a finite extension $\mathbf{F}^{\prime}$ of $\mathbf{F}$, a point $x$ in $X\left(\mathbf{F}^{\prime}\right)$, and a subgroup $R$ of $A$ such that the rank of $R$ is $\geqslant k-d$ and every element of $R$ is defined at the point $x$ and fixes it. 
Proof. Changing $\mathbf{F}$ in a finite extension, $X$ in one of its irreducible components, and $A$ in a finite-index subgroup, we may assume that $X$ is absolutely irreducible. Fix an algebraic closure $\overline{\mathbf{F}}$ of $\mathbf{F}$. We may assume that $d$ is positive, since otherwise $X$ is just one point. We fix a system of generators for $A$ and an open subset $U$ of $X$ such that, on $U$, every generator restricts to an open immersion $\left.s\right|_{U}: U \rightarrow X$ (see $\S 7.2 .1$ ). Assume by contradiction that the regular stabilizer $R_{x}$ of every point of $X(\overline{\mathbf{F}})$ has rank at most $l$, with $l<k-d$. Denote by $\alpha$ the ratio $q /(q-1)$ with $q=k-l>d$; we have $1<\alpha<d /(d-1)$. Let $\mathbf{F}^{\prime}$ be a finite extension of $\mathbf{F}$ and $\left\{x_{i}\right\}_{i}$ be a set of representatives of the regular orbits of $U\left(\mathbf{F}^{\prime}\right)$. From assertion (3) of Proposition 7.9, we obtain

$$
\left|\partial \Omega_{x_{i}}\right|=\left|\mathrm{ev}_{x_{i}}\left(\partial \Omega_{x_{i}}\right)\right|=\left|E_{x_{i}}\left(\mathbf{F}^{\prime}\right)\right|,
$$

and Lemma 7.11 provides a constant $c>0$ such that

$$
\left|U\left(\mathbf{F}^{\prime}\right)\right|=\sum_{i}\left|\Omega_{x_{i}}\right| \leqslant c \sum_{i}\left|\partial \Omega_{x_{i}}\right|^{\alpha}=c \sum_{i}\left|E_{x_{i}}\left(\mathbf{F}^{\prime}\right)\right|^{\alpha} \leqslant c\left(\sum_{i}\left|E_{x_{i}}\left(\mathbf{F}^{\prime}\right)\right|\right)^{\alpha} .
$$

From Lang-Weil estimates, one derives

$$
\left|\mathbf{F}^{\prime}\right|^{d} \leqslant c_{U}\left|\mathbf{F}^{\prime}\right|^{d-1 / 2}+c^{s t}\left(b_{E}\left|\mathbf{F}^{\prime}\right|^{d-1}+c_{E}\left|\mathbf{F}^{\prime}\right|^{d-3 / 2}\right)^{\alpha} .
$$

This provides a contradiction if $\left|\mathbf{F}^{\prime}\right|$ is large, because $(d-1) \alpha<d$.

\subsection{Invariant polydisks for groups with property $\left(\tau^{\infty}\right)$}

Let $X_{\mathbf{Q}_{p}}$ be an absolutely irreducible projective variety. Assume that $X$ is defined over $\mathbf{Z}_{p}$, that $\Gamma$ is a finitely generated subgroup of $\operatorname{Bir}\left(X_{\mathbf{Z}_{p}}\right)$ with a finite symmetric set of generators $S$, and that $\left(X_{\mathbf{Z}_{p}}, \Gamma\right)$ is a good model over $\mathbf{Z}_{p}$.

Theorem 7.13. Assume that $\Gamma$ has property $\left(\tau^{\infty}\right)$. There exist a finite extension $K$ of $\mathbf{Q}_{p}$, a finite-index subgroup $\Gamma_{0}$ of $\Gamma$, and a Tate analytic diffeomorphism $\varphi$ from the unit polydisk $\mathcal{U}=\left(O_{K}\right)^{d} \subset K^{d}$ to an open subset $\mathcal{V}$ of $X(K)$ such that $\mathcal{V}$ is $\Gamma_{0}$-invariant and the action of $\Gamma_{0}$ on $\mathcal{V}$ is conjugate, via $\varphi$, to a subgroup of $\operatorname{Diff}^{\mathrm{an}}(\mathcal{U})$. Moreover, one can choose this polydisk in the complement of any given proper Zariski closed subset of the generic fiber.

The following proof constructs $\Gamma_{0}$ as a regular stabilizer $R_{x}$.

Proof. Given $g \in \operatorname{Bir}\left(X_{\mathbf{Z}_{p}}\right)$, recall that $B_{\mathbf{Z}_{p}, g}$ denotes the complement of the points of $X_{\mathbf{Z}_{p}}$ around which $g$ is an open immersion (see $\left.\S 3.1 .2\right)$. Since $\left(X_{\mathbf{Z}_{p}}, \Gamma\right)$ is a good model over $\mathbf{Z}_{p}$, the singular locus of the scheme $X_{\mathbf{Z}_{p}}$ and the sets $B_{\mathbf{Z}_{p}, g}$, for $g$ in $S$, have 
codimension $\geqslant 1$ in $X_{\mathbf{Z}_{p}}$ and in the special fiber too (see (ii) and (iii) in §3.1.2). Denote by $\operatorname{Sing}\left(X_{\mathbf{Z}_{p}}\right)$ the singular locus of $X_{\mathbf{Z}_{p}}$, and set

$$
U_{\mathbf{z}_{p}}:=X_{\mathbf{Z}_{p}} \backslash\left(\operatorname{sing}\left(X_{\mathbf{Z}_{p}}\right) \cup\left(\bigcup_{s \in S} B_{\mathbf{Z}_{p}, s}\right)\right) .
$$

Let $X_{\mathbf{F}_{p}}$ be the special fiber. By assumption, $U_{\mathbf{Z}_{p}} \cap X_{\mathbf{F}_{p}}$ is a non-empty Zariski open subset of $X_{\mathbf{F}_{p}}$; let $U$ be any open subset of $U_{\mathbf{Z}_{p}} \cap X_{\mathbf{F}_{p}}$ (for instance, take for $U$ the complement of a given divisor). Observe that, for any $s \in S$, the map $\left.s\right|_{U_{\mathbf{z}_{p}}}: U_{\mathbf{z}_{p}} \rightarrow X_{\mathbf{z}_{p}}$ is an open immersion; hence, $\left.s\right|_{U}: U \rightarrow X_{\mathbf{F}_{p}}$ is also an open immersion.

By Theorem 7.10, there exist a finite field extension $\mathbf{F}^{\prime}$ of $\mathbf{F}_{p}$ and a point $x \in U\left(\mathbf{F}^{\prime}\right)$ such that the regular stabilizer $R_{x}$ of $x$ is a finite-index subgroup of $\Gamma$. Let $K$ be a finite extension of $\mathbf{Q}_{p}$ whose residue field is $\mathbf{F}^{\prime}$.

Every element $g$ of $R_{x}$ is a regular morphism on a neighborhood of $x$ and fixes $x$. Denote by $\mathcal{W}$ the set of $K$-points $y$ in $X_{K}$ whose specialization in the special fiber $X_{\mathbf{F}^{\prime}}$ coincides with $x$. By Proposition 3.4, one can find an analytic diffeomorphism $\varphi$ from the unit polydisk $\mathcal{U}=\left(O_{K}\right)^{d} \subset K^{d}$ to an open subset $\mathcal{V} \subset \mathcal{W}$ such that $\mathcal{V}$ is $R_{x}$-invariant and the action of $R_{x}$ on $\mathcal{V}$ is conjugate, via $\varphi$, to a subgroup of $\operatorname{Diff}^{\text {an }}(\mathcal{U})$.

Similarly, Theorem 7.12 provides invariant polydisks for subgroups of rank $l \geqslant k-$ $\operatorname{dim}(X)$ when $\Gamma$ is a free abelian group of rank $k$.

\subsection{Groups of birational transformations and finite-index subgroups}

\subsubsection{Groups of birational transformations}

A group $\Gamma$ is linear over the field $\mathbf{k}$ if $\Gamma$ is isomorphic to a subgroup of $\mathrm{GL}_{n}(\mathbf{k})$ for some $n \geqslant 1$ (see [39]). Similarly, a group $\Gamma$ is a group of birational transformations over the field $\mathbf{k}$ if $\Gamma$ is isomorphic to a subgroup of $\operatorname{Bir}\left(X_{\mathbf{k}}\right)$ for some algebraic variety defined over $\mathbf{k}$.

Example 7.14. Linear groups over $\mathbf{k}$ are groups of birational transformations over $\mathbf{k}$. Every finite group is a group of automorphisms of some complex irreducible curve (see $\left[36\right.$, Theorem $\left.6^{\prime}\right]$ ). The modular group $\operatorname{Mod}(g)$ of a closed, orientable surface of genus $g \geqslant 3$ and the group Out $\left(\mathbb{F}_{g}\right)$ are groups of birational transformations in dimension $\leqslant 6 g$ over $\mathbf{C}$, but Out $\left(\mathbb{F}_{g}\right)$ is not a linear group if $g \geqslant 4$ (see $\S 6$ and [50], [33]).

\subsubsection{Malcev and Selberg properties}

In characteristic zero, linear groups satisfy Malcev and Selberg properties: every finitely generated linear group is residually finite and contains a torsion-free, finite-index sub- 
group. One does not know whether groups of birational transformations share the same properties (see [14] and [21]). The following result implies Theorem $\mathrm{C}$ of the introduction.

THEOREM 7.15. Let $\Gamma$ be a discrete group with property $\left(\tau^{\infty}\right)$. If $\Gamma$ is a group of birational transformations over a field $\mathbf{k}$ of characteristic zero, then $\Gamma$ is residually finite and contains a torsion-free, finite-index subgroup.

Proof. Since $\Gamma$ has property $\left(\tau^{\infty}\right)$, it is finitely generated (see $\S 7.1$ and Proposition 7.3); fix a finite symmetric set of generators $S$ for $\Gamma$, and an embedding of $\Gamma$ in the group of birational transformations of a smooth projective variety $X$ (over an algebraically closed field $\mathbf{k}$ of characteristic zero). Pick an element $f$ in $\Gamma \backslash\{\operatorname{Id}\}$ and denote by $\operatorname{Fix}(f)$ the proper Zariski closed set of fixed points of $f$; more precisely, $\operatorname{Fix}(f) \subset X$ is defined as the Zariski closure of the subset of the domain of definition of $f$ defined by the equation $f(z)=z$. By Proposition 3.2, one can find a prime number $p \geqslant 3$, and a good model $\Gamma \subset \operatorname{Bir}\left(X_{\mathbf{Z}_{p}}\right)$ for $(X, \Gamma)$, such that the special fiber $X_{\mathbf{F}_{p}}$ of $X_{\mathbf{Z}_{p}}$ is not contained in $\operatorname{Fix}(f)$.

Choose a Zariski open subset $U$ of $X_{\mathbf{F}_{p}}$ which is contained in the complement of $\operatorname{Fix}(f)$ and of the sets $B_{\mathbf{Z}_{p}, s}$, for $s \in S$. We now apply Theorem 7.10. Since $\Gamma$ has property $\left(\tau^{\infty}\right)$, one can find an extension $\mathbf{F}^{\prime}$ of the residue field $\mathbf{F}_{p}$, and a point $x \in U\left(\mathbf{F}^{\prime}\right)$, for which the regular stabilizer $R_{x}$ has finite index in $\Gamma$. By construction, $R_{x}$ does not contain $f$. This shows that $\Gamma$ is residually finite.

Let $\mathcal{U}$ be the polydisk $\left(O_{K}\right)^{d}$. To prove the second assertion, keep the same notation and apply Theorem 7.13. This provides an $R_{x}$-invariant subset $\mathcal{V}$ and an analytic diffeomorphism $\varphi: \mathcal{U} \rightarrow \mathcal{V}$ such that, after conjugacy by $\varphi, R_{x}$ acts by Tate analytic diffeomorphisms on $\mathcal{U}$. Then, there exists a finite-index subgroup $R_{x}^{\prime}$ of $R_{x}$ such that every element $g \in R_{x}^{\prime}$ corresponds to a power series

$$
g(z)=A_{0}+A_{1}(z)+\sum_{k \geqslant 2} A_{k}(z)
$$

where each $A_{i}$ is homogeneous of degree $i, A_{0}$ is zero modulo $p^{2}$ and $A_{1}$ is the identity modulo $p$. After conjugation by $z \mapsto p z$, the Bell-Poonen theorem (Theorem 2.4) can be applied to $g$. Thus, Corollary 2.5 shows that $R_{x}^{\prime}$ is torsion-free.

\subsubsection{Central extensions and simple groups}

Fix two positive integers $q$ and $n$, with $q \neq 1,2,4$ and $n \geqslant 2$. Consider the group $\operatorname{Sp}_{2 n}(\mathbf{Z})$, and the central extension

$$
0 \longrightarrow \mathbf{Z} / q \mathbf{Z} \longrightarrow \Gamma \longrightarrow \mathrm{Sp}_{2 n}(\mathbf{Z}) \longrightarrow 1
$$


which is obtained from the universal cover

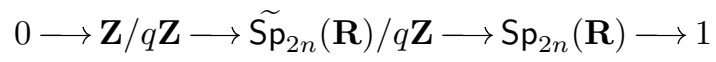

by taking the quotient with respect to the subgroup $q \mathbf{Z}$ of the center $\mathbf{Z} \subset \widetilde{\operatorname{Sp}}_{2 n}(\mathbf{R})$. Since $n \geqslant 2, \mathrm{Sp}_{2 n}(\mathbf{Z})$ has Kazhdan property $(\mathrm{T})$ (see [40]). Since $q$ does not divide 4 , the image of $4 \mathbf{Z}$ in the center $\mathbf{Z} / q \mathbf{Z}$ of $\Gamma$ is non-trivial and is contained in every finite-index subgroup of $\Gamma$ (see [25]); consequently, $\Gamma$ does not contain any torsion-free finite-index subgroup.

COROLlaRY 7.16. The group $\mathrm{Sp}_{2 n}(\mathbf{Z})$ is a group of birational transformations over the field $\mathbf{Q}$ but, if $n \geqslant 2$, there is a finite cyclic central extension $\Gamma$ of $\mathbf{S p}_{2 n}(\mathbf{Z})$ that does not act faithfully by birational transformations in characteristic zero.

In particular, the property " $\Gamma$ is a group of birational transformations" is not stable under finite central extensions. Similar examples can be derived from [53] and [56]. The following corollary shows that the simple groups constructed in [30] and [20] do not act non-trivially by birational transformations.

Corollary 7.17. If $\Gamma$ is an infinite, simple, discrete group with property $\left(\tau^{\infty}\right)$, and $X$ is a complex projective variety, every homomorphism $\Gamma \rightarrow \operatorname{Bir}(X)$ is trivial.

Proof. A non-trivial homomorphism $\Gamma \rightarrow \operatorname{Bir}(X)$ is an embedding, as $\Gamma$ is simple. If such an embedding exists, $\Gamma$ contains non-trivial finite-index subgroups, contradicting the simplicity of $\Gamma$.

\section{Birational actions of lattices on quasi-projective varieties}

In this section, we prove Theorem B, and a corollary which concerns birational actions of the lattice $\mathrm{SL}_{n}(\mathbf{Z})$ and its finite-index subgroups.

\subsection{Lattices in higher-rank Lie groups}

Let $\mathrm{S} \subset \mathrm{GL}_{m}$ be an algebraic subgroup of $\mathrm{GL}_{m}$ defined over the field of rational numbers $\mathbf{Q}$ (see [9]). We make the following assumptions:

(i) $\mathbf{S}$ is almost $\mathbf{R}$-simple (the Lie algebra $\mathfrak{g}_{\mathbf{R}}$ of $\mathbf{S}(\mathbf{R})$ is simple);

(ii) as an algebraic group, $\mathbf{S}$ is connected and simply connected (equivalently, $\mathbf{S}(\mathbf{C})$ is a simply connected manifold);

(iii) the real rank of $\mathrm{S}$ is greater than 1 (see $\S 1.3$ );

(iv) the lattice $\Gamma=S(\mathbf{Z})$ of $\mathbf{S}(\mathbf{R})$ is not cocompact (i.e. $\operatorname{rank}_{\mathbf{Q}}(\mathrm{S})>0$ ).

We refer to [58], [54, §7.4], and [57] for a good introduction to the following result, and for references to the literature and original contributions. 
THEOREM 8.1. Let $\mathrm{S}$ be an algebraic subgroup of $\mathrm{GL}_{m}$, defined over the field $\mathbf{Q}$, with the above four properties. Then $\mathrm{S}(\mathbf{Z})$ satisfies the strong approximation and congruence subgroup properties.

This means that the closure of $\mathbf{S}(\mathbf{Z})$ has finite index in $\prod_{q} \mathbf{S}\left(\mathbf{Z}_{q}\right)$, and that every finite-index subgroup of $\mathbf{S}(\mathbf{Z})$ contains a congruence subgroup $\{B \in \mathbf{S}(\mathbf{Z}): B \equiv \operatorname{Id}(\bmod m)\}$ for some integer $m$. In other words, the profinite completion of $\mathbf{S}(\mathbf{Z})$ coincides with a finite-index subgroup of $\prod_{q} \mathrm{~S}\left(\mathbf{Z}_{q}\right)$.

Lemma 8.2. Let $\mathbf{S}$ be an algebraic subgroup of $\mathrm{GL}_{m}$ defined over $\mathbf{Q}$, that satisfies the above four properties. If $\Gamma$ is a finite-index subgroup of $\mathbf{S}(\mathbf{Z})$, then $\mathbf{S}\left(\mathbf{Z}_{p}\right)$ is a virtual pro-p completion of $\Gamma$.

Proof. (See also §4.2.3.) According to [61] (Theorems 5 and 34, and the corollary to Lemma 64) and to the above Theorem 8.1, there is a prime $q_{0}$ such that $\mathbf{S}\left(\mathbf{F}_{q}\right)$ is a perfect group and $\mathbf{S}(\mathbf{Z})$ is dense in $\mathbf{S}\left(\mathbf{Z}_{q}\right)$ for every prime $q \geqslant q_{0}$.

Let $\Gamma$ be a finite-index subgroup of $\mathbf{S}(\mathbf{Z})$. Let $m$ be a positive integer such that $p$ divides $m$, every prime $q<q_{0}$ divides $m$, and the congruence subgroup

$$
\Gamma_{m}:=\{B \in \mathrm{S}(\mathbf{Z}): B \equiv \operatorname{Id}(\bmod m)\}
$$

is contained in $\Gamma$. Let $F$ be the set of prime divisors of $m$.

Denote by $G_{q}$ the closure of $\Gamma_{m}$ in $\mathrm{S}\left(\mathbf{Z}_{q}\right)$; the profinite completion of $\Gamma_{m}$ is $\prod_{q} G_{q}$. The first congruence subgroup of $\mathbf{S}\left(\mathbf{Z}_{q}\right)$ is an analytic pro- $q$ group, thus, if $q \in F \backslash\{p\}$, $G_{q}$ is a pro- $q$ group and every morphism to a $p$-group is trivial. Similarly, if $q \notin F$, every morphism from $G_{q}=\mathrm{S}\left(\mathbf{Z}_{q}\right)$ to a $p$-group factors through the quotient $\mathrm{S}\left(\mathbf{F}_{q}\right)$, and is trivial because $\mathbf{S}\left(\mathbf{F}_{q}\right)$ is perfect. This shows that the pro- $p$ completion of $\Gamma_{m}$ is $G_{p}$, and that $\mathrm{S}\left(\mathbf{Z}_{p}\right)$ is a virtual pro- $p$ completion of $\Gamma$.

\subsection{Minimal homogeneous spaces (see [65, p. 187] and [63])}

Given a simple complex Lie algebra $\mathfrak{s}$, one denotes by $\delta(\mathfrak{s})$ the minimal codimension of its proper Lie subalgebras $\mathfrak{p}<\mathfrak{s}$. If $\mathrm{S}$ is a complex connected algebraic group with Lie algebra equal to $\mathfrak{s}$, then $\delta(\mathfrak{s})$ is equal to the minimal dimension $\delta(\mathrm{S})$ of a homogeneous variety $V=\mathrm{S} / P$ with $\operatorname{dim}(V)>0$. Such a maximal group $P$ is the stabilizer of a point $m \in V$; it is a parabolic subgroup of $\mathbf{S}$ (see [65, p. 187]). If $\mathfrak{s}$ (resp. S) is defined over a subfield of $\mathbf{C}$, we use the same notation $\delta(\mathfrak{s})(\operatorname{resp} . \delta(\mathrm{S}))$ to denote $\delta(\mathfrak{s} \otimes \mathbf{C})$.

This dimension $\delta(\mathrm{S})$ has been computed for all complex, simple and connected algebraic groups (see [63] for instance). The results are summarized in Table 1, from which one sees that $\delta(\mathfrak{s}) \geqslant \operatorname{rank}_{\mathbf{C}}(\mathfrak{s})$ with equality if and only if $\mathfrak{s}$ is $\mathfrak{s l}_{\delta(\mathfrak{s})+1}(\mathbf{C})$. 


\begin{tabular}{|c|c|c|c|}
\hline Lie algebra & dimension & $\begin{array}{c}\text { dimension } \\
\text { of the minimal } \\
\text { representation }\end{array}$ & $\begin{array}{c}\text { dimension } \\
\text { of the smallest } \\
\text { homogeneous space }\end{array}$ \\
\hline \hline $\mathfrak{s l}_{k}(\mathbf{C}), k \geqslant 2$ & $k^{2}-1$ & $k$ & $k-1$ \\
\hline $\mathfrak{s o}_{k}(\mathbf{C}), k \geqslant 7$ & $\frac{1}{2} k(k-1)$ & $k$ & $k-2$ \\
\hline $\mathfrak{s p}_{2 k}(\mathbf{C}), k \geqslant 2$ & $k(2 k+1)$ & $2 k$ & $2 k-1$ \\
\hline $\mathfrak{e}_{6}(\mathbf{C})$ & 78 & 27 & 14 \\
\hline $\mathfrak{e}_{7}(\mathbf{C})$ & 133 & 56 & 57 \\
\hline $\mathfrak{e}_{8}(\mathbf{C})$ & 248 & 248 & 15 \\
\hline $\mathfrak{f}_{4}(\mathbf{C})$ & 52 & 26 & 5 \\
\hline $\mathfrak{g}_{2}(\mathbf{C})$ & 14 & 7 & \\
\hline
\end{tabular}

Table 1. Minimal dimensions of faithful representations and minimal homogeneous spaces.

Remark 8.3. Here are a few comments on Table 1 . The inequality $\delta(\mathfrak{s}) \geqslant \operatorname{rank}_{\mathbf{C}}(\mathfrak{s})$ may be obtained with the following argument. Choose a maximal torus $T$ in $\mathbf{S}$. Since $\mathbf{S}$ is almost simple, $T$ acts on $V=\mathrm{S} / P$ with a finite kernel, and hence the isotropy group in $T$ of a general point of $V$ is finite; thus, $\operatorname{dim}(V) \geqslant \operatorname{dim}(T)$.

The algebra $\mathfrak{s l}_{k}(\mathbf{C})$ has two representations of minimal dimension (the standard representation on $\mathbf{C}^{k}$ and its dual); likewise, $\mathrm{SL}_{k}(\mathbf{C})$ has two minimal homogeneous spaces. (The other simple complex Lie algebras have a unique minimal representation, up to isomorphism).

The group $\mathrm{SO}_{5}(\mathbf{C})$ is isogenous to $\mathrm{Sp}_{4}(\mathbf{C})$ and acts on $\mathbb{P}^{3}$ (the space of lines in the smooth quadric $Q \subset \mathbb{P}^{4}$ is isomorphic to $\left.\mathbb{P}^{3}\right)$. Similarly, $\mathrm{SO}_{6}(\mathbf{C})$ is isogenous to $\mathrm{SL}_{4}(\mathbf{C})$ and acts on $\mathbb{P}^{3}$ too.

\subsection{Proof of Theorem $B$}

Changing $S$ into a finite cover, and $\Gamma$ into its pre-image under the covering homomorphism, we may assume that the semisimple algebraic group $S$ is simply connected. Identify $\Gamma$ with its image in $\operatorname{Bir}(X)$, and choose a good $p$-adic model for $(X, \Gamma)$, as in Proposition 3.2 .

The group $\Gamma$ is a lattice in the higher rank, almost simple Lie group $\mathbf{S}(\mathbf{R})$. As such, $\Gamma$ has Kazhdan property (T) (see [40, Chapters 2 and 3$]$ ); hence, it has property $\left(\tau^{\infty}\right)$. 
According to Theorem 7.13, there is a finite-index subgroup $\Gamma_{0}$ in $\Gamma$, a field extension $K$ of $\mathbf{Q}_{p}$ and an analytic polydisk $\mathcal{U} \subset X(K)$ which is $\Gamma_{0}$-invariant, and on which $\Gamma_{0}$ acts by Tate analytic diffeomorphisms.

We also know from Lemma 8.2 that $\mathbf{S}\left(\mathbf{Z}_{p}\right)$ is a virtual pro- $p$ completion of $\Gamma_{0}$. By Theorem 2.11, there exists a finite-index subgroup $\Gamma_{1}$ of $\Gamma_{0}$ such that the analytic action of $\Gamma_{1}$ on the polydisk $\mathcal{U}$ extends to an analytic action of its closure $G_{1}=\bar{\Gamma}_{1}$, an open subgroup of the $p$-adic group $\mathbf{S}\left(\mathbf{Z}_{p}\right)$.

Let $o$ be a point of $\mathcal{U}$ which is not fixed by $G_{1}$; the stabilizer of $o$ is a closed subgroup $P$ of $G_{1}$ : its Lie algebra determines a subalgebra of $\mathfrak{s}$ of codimension at most $\operatorname{dim}(X)$. If $\operatorname{dim}(X)<\delta(\mathrm{S})$, then $P$ is a finite-index subgroup of $G_{1}$, and the action of $\Gamma_{1}$ on $X$ factors through a finite group. Thus,

$$
\operatorname{dim}(X) \geqslant \delta(\mathrm{S}) \geqslant \operatorname{rank}_{\mathbf{R}}(\mathrm{S})
$$

If $\operatorname{dim}(X)=\operatorname{rank}_{\mathbf{R}}(\mathrm{S})$, then $\delta(\mathrm{S})=\operatorname{rank}_{\mathbf{R}}(\mathrm{S})$ and $\mathfrak{s}=\mathfrak{s l}_{n}$ with $n=\operatorname{dim}(X)+1$.

Remark 8.4. The inequality (8.1) is stronger than $\operatorname{dim}(X) \geqslant \operatorname{rank}_{\mathbf{R}}(\mathrm{S})$. For instance, if $\Gamma$ is a non-uniform lattice in $\mathrm{F}_{4}$, then $\Gamma$ does not act faithfully by birational transformations in dimension $\leqslant 14$.

Corollary 8.5. Let $\Gamma$ be a finite-index subgroup of $\mathrm{SL}_{n}(\mathbf{Z})$, with $n \geqslant 3$. If $\Gamma$ acts by birational transformations on an irreducible complex projective variety $X$, then either the image of $\Gamma$ in $\operatorname{Bir}(X)$ is finite, or $\operatorname{dim}(X) \geqslant n-1$. Moreover, if the image is infinite and $\operatorname{dim}(X)=n-1$, then $X$ is rational, and the action of $\Gamma$ on $X$ is birationally conjugate to a linear projective action of $\Gamma$ on $\mathbb{P}^{n-1}$.

Proof. Let $\Gamma_{m}$ be a principal congruence subgroup which is contained in $\Gamma$, with $m \equiv 0(\bmod 3) ;$ then $\Gamma_{m}$ is torsion-free. The kernel of the action of $\Gamma_{m}$ on $X$ is either trivial, or a finite-index subgroup, because every infinite normal subgroup of $\Gamma_{m}$ has finite index. Thus, we may now assume that $\Gamma_{m}$ acts faithfully on $X$ by birational transformations. Theorem B implies that $\operatorname{dim}(X) \geqslant n-1$. In case of equality, there is a good, $p$-adic model of $(X, \Gamma)$ such that a finite-index subgroup of $\Gamma$ preserves a $p$-adic polydisk and acts by analytic diffeomorphisms on it. Then, Theorem 5.1 shows that there is a birational, $\Gamma$-equivariant mapping $\tau: X-\rightarrow \mathbb{P}^{n-1}$, where the action of $\Gamma$ on $\mathbb{P}^{n-1}$ is by linear projective automorphisms. 


\section{Appendix}

\subsection{Proof of Proposition 3.2}

As explained in $§ 3.1$, there exists a subring $R$ of $\mathbf{k}$, which is finitely generated over $\mathbf{Z}$, such that $X$ and the birational transformations $s \in S$ are defined over $R$. This means that there exists a projective scheme $X_{R} \rightarrow \operatorname{Spec}(R)$ such that $X=X_{R} \times_{\operatorname{Spec}(R)} \operatorname{Spec}(\mathbf{k})$. Let $\pi: X_{R} \rightarrow \operatorname{Spec}(R)$ be such a model, with generic fiber $X_{K}(K$ is the fraction field of $R)$.

Lemma 9.1. There exists a non-empty, affine, open subset $U$ of $\operatorname{Spec}(R)$ such that the following conditions are satisfied:

(i) $U$ is of finite type over $\operatorname{Spec}(\mathbf{Z})$;

(ii) for every point $y \in U$, the fiber $X_{y}$ is absolutely irreducible and

$$
\operatorname{dim}_{K(y)} X_{y}=\operatorname{dim}_{K} X_{K}
$$

where $K(y)$ is the residue field at $y$;

(iii) for every $s \in S$ and every $y \in U$, the fiber $X_{y}$ is not contained in $B_{R, s}$.

Proof. (See [7, Proposition 4.3].) To prove the lemma, we shall use the following fact: For any integral affine $\operatorname{scheme} \operatorname{Spec}(A)$ of finite type over $\operatorname{Spec}(\mathbf{Z})$ and any nonempty open subset $V_{1}$ of $\operatorname{Spec}(A)$, there exists an affine open subset $V_{2}$ of $V_{1}$ which is of finite type over $\operatorname{Spec}(\mathbf{Z})$. Indeed, we may pick any non-zero element $f \in I$, where $I$ is the ideal of $A$ that defines the closed subset $\operatorname{Spec}(A) \backslash V$ and set $U:=\operatorname{Spec}(A) \backslash\{f=0\}$. Then $U=\operatorname{Spec}(A[1 / f])$ is of finite type over $\operatorname{Spec}(\mathbf{Z})$.

Since $X_{K}$ is absolutely irreducible, [37, Proposition 9.7.8] gives an affine open subset $V$ of $\operatorname{Spec}(R)$ such that $X_{y}$ is absolutely irreducible for every $y \in V$. We may suppose that $V$ is of finite type over $\operatorname{Spec}(\mathbf{Z})$. By generic flatness (see [37, Theorem 6.9.1]), we may change $V$ in a smaller subset and suppose that the restriction of $\pi$ to $\pi^{-1}(V)$ is flat.

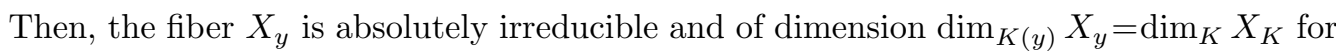
every point $y \in V$.

For $s \in S$, denote by $B_{K, s}$ the complement of the points in $X_{K}$ around which $s$ is an open immersion. Observe that $B_{K, s}$ is exactly the generic fiber of $\left.\pi\right|_{B_{R, s}}: B_{R, s} \rightarrow \operatorname{Spec}(R)$. By generic flatness, there exists a non-empty, affine, open subset $U_{s}$ of $V$ such that the restriction of $\pi$ to every irreducible component of $\left(\left.\pi\right|_{B_{R, s}}\right)^{-1}\left(U_{s}\right)$ is flat. Let $U$ be the intersection of the open subsets $U_{s}$, for $s$ in $S$; then, shrink $U$ to suppose that $U$ is of finite type over $\operatorname{Spec}(\mathbf{Z})$. Since

$$
\operatorname{dim}_{K(y)}\left(B_{R, s} \cap X_{y}\right)=\operatorname{dim}_{K}\left(B_{K, s}\right)<\operatorname{dim}_{K} X_{K}=\operatorname{dim}_{K(y)} X_{y}
$$

for every $s \in S$ and $y \in U$, the fiber $X_{y}$ is not contained in $B_{R, s}$. 
By Lemma 9.1, we may replace $\operatorname{Spec}(R)$ by $U$ and assume that

- for every $y \in \operatorname{Spec}(R)$, the fiber $X_{y}$ is absolutely irreducible;

- for every $s \in S$ and $y \in \operatorname{Spec}(R)$, the fiber $X_{y}$ is not contained in $B_{R, s}$.

Since $R$ is integral and finitely generated over $\mathbf{Z}$, by Lemma 3.1 there exist infinitely many primes $p \geqslant 3$ such that $R$ can be embedded into $\mathbf{Z}_{p}$. This induces an embedding $\operatorname{Spec}\left(\mathbf{Z}_{p}\right) \rightarrow \operatorname{Spec}(R)$. Set $X_{\mathbf{Z}_{p}}:=X_{R} \times_{\operatorname{Spec}(R)} \operatorname{Spec}\left(\mathbf{Z}_{p}\right)$. All fibers $X_{y}$, for $y \in \operatorname{Spec}(R)$, are absolutely irreducible and of dimension $d$; hence, the special fiber $X_{\mathbf{F}_{p}}$ of $X_{\mathbf{Z}_{p}} \rightarrow \operatorname{Spec}\left(\mathbf{Z}_{p}\right)$ is absolutely irreducible and of dimension $d=\operatorname{dim}(X)$. Since $B_{\mathbf{Z}_{p}, s} \cap X_{\mathbf{F}_{p}} \subset B_{R, s} \cap X_{\mathbf{F}_{p}}$ for every $s \in S$, the fiber $X_{\mathbf{F}_{p}}$ is not contained in $B_{\mathbf{Z}_{p}, s}$. Thus, $X_{\mathbf{Z}_{p}}$ provides a good model for $(X, \Gamma)$.

\subsection{From fixed points to invariant polydisks}

We now prove Proposition 3.4; the notation is from $\S 3.2 .2$. Since $X_{O_{K}}$ is projective, there exists an embedding $\psi: X_{O_{K}} \rightarrow \mathbb{P}_{O_{K}}^{N}$ defined over $O_{K}$. On the projective space $\mathbb{P}^{N}(K)$, there is a metric dist ${ }_{p}$ defined by

$$
\operatorname{dist}_{p}\left(\left[x_{0}: \ldots: x_{N}\right],\left[y_{0}: \ldots: y_{N}\right]\right)=\frac{\max _{i \neq j}\left|x_{i} y_{j}-x_{j} y_{i}\right|_{p}}{\left(\max _{i}\left|x_{i}\right|_{p}\right)\left(\max _{j}\left|y_{j}\right|_{p}\right)}
$$

for all points $\left[x_{0}: \ldots: x_{N}\right],\left[y_{0}: \ldots: y_{N}\right] \in \mathbb{P}^{N}(K)$. Via the embedding $\left.\psi\right|_{X(K)}: X_{K}(K) \rightarrow \mathbb{P}_{K}^{N}$, $\operatorname{dist}_{p}$ restricts to a metric dist $p, \psi$ on $X_{K}(K)$. This metric does not depend on the choice of the embedding $\psi$; thus, we simply write dist $_{p}$ instead of dist ${ }_{p, \psi}$.

Lemma 9.2. For $w, z \in X_{K}(K) \operatorname{dist}_{p}(w, z)<1$ if and only if the reductions $r(w)$ and $r(z)$ coincide.

Proof. Set $\psi(w)=\left[x_{0}: \ldots: x_{N}\right]$ and $\psi(z)=\left[y_{0}: \ldots: y_{N}\right]$, where the coordinates $x_{i}$ and $y_{i}$ are in $O_{K}$ and satisfy $\max _{i}\left|x_{i}\right|_{p}=\max _{i}\left|y_{i}\right|_{p}=1$. Then,

$$
\psi(r(w))=\left[\bar{x}_{0}: \ldots: \bar{x}_{N}\right] \text { and } \psi(r(z))=\left[\bar{y}_{0}: \ldots: \bar{y}_{N}\right]
$$

where $\bar{x}_{i}$ and $\bar{y}_{i}$ denote the images of $x_{i}$ and $y_{i}$ in the residue field $\mathbf{F}=O_{K} / \mathbf{m}_{K}$. By definition,

$$
\operatorname{dist}_{p}\left(\left[x_{0}: \ldots: x_{N}\right],\left[y_{0}: \ldots: y_{N}\right]\right)=\max _{i \neq j}\left|x_{i} y_{j}-x_{j} y_{i}\right|_{p}
$$

If $r(w)=r(z)$, we have $\bar{x}_{i}=\bar{y}_{i}$ for all indices $i$; thus,

$$
\left|x_{i} y_{j}-x_{j} y_{i}\right|_{p}=\left|\left(x_{i}-y_{i}\right) y_{j}-\left(x_{j}-y_{j}\right) y_{i}\right|_{p}<1
$$


and $\operatorname{dist}_{p}(w, z)<1$. Now, suppose that $r(w) \neq r(z)$. Assume, first, that there exists an index $i$, say $i=0$, with $\bar{x}_{i} \bar{y}_{i} \neq 0$. Replacing each $x_{j}$ by $x_{j} / x_{0}$ and each $y_{j}$ by $y_{j} / y_{0}$, we get $x_{0}=y_{0}=1$. Since $r(w) \neq r(z)$, there exists $j \geqslant 1$ with $\bar{x}_{j} \neq \bar{y}_{j}$. It follows that

$$
\operatorname{dist}_{p}(w, z) \geqslant\left|x_{j} y_{0}-x_{0} y_{j}\right|_{p}=\left|x_{j}-y_{j}\right|_{p}=1 \text {. }
$$

To conclude, suppose that $\bar{x}_{i} \bar{y}_{i}=0$ for all $i \in\{0, \ldots, N\}$. Pick two indices $i$ and $j$ such that $\bar{x}_{i} \neq 0$ and $\bar{y}_{j} \neq 0$; thus, $\bar{y}_{i}=0$ and $\bar{x}_{j}=0$, and $\operatorname{dist}_{p}(w, z) \geqslant\left|x_{i} y_{j}-x_{j} y_{i}\right|_{p}=1$.

Recall that $x$ is a smooth point in $X(\mathbf{F})$ and $\mathcal{V}$ is the open subset of $X_{K}(K)$ consisting of points $z \in X_{K}(K)$ satisfying $r(z)=x$. With suitable homogeneous coordinates, $x$ is the point $[1: 0: \ldots: 0] \in \mathbb{P}_{\mathbf{F}}^{N}$. Then, the open set $\mathcal{V}$ is contained in the unit polydisk

$$
B:=\left\{\left[1: z_{1}: \ldots: z_{N}\right]: z_{i} \in O_{K} \text { for all } i=1, \ldots, N\right\}
$$

Recall from $\S 2.1 .1$ that a map $\varphi$ from the unit polydisk $\mathcal{U}=O_{K}^{d} \subset K^{d}$ to $B$ is analytic if we can find elements $\varphi_{i}, 1 \leqslant i \leqslant N$, of the Tate algebra $O_{K}\left\langle\mathbf{x}_{1}, \ldots, \mathbf{x}_{d}\right\rangle$, such that

$$
\varphi\left(x_{1}, \ldots, x_{d}\right)=\left[1: \varphi_{1}\left(x_{1}, \ldots, x_{d}\right): \ldots: \varphi_{N}\left(x_{1}, \ldots, x_{d}\right)\right]
$$

Proposition 9.3. There exists a one-to-one analytic diffeomorphism $\varphi$ from the unit polydisk $\mathcal{U}=\left(O_{K}\right)^{d} \subset K^{d}$ to $\mathcal{V}$.

Proof. Consider the affine chart $\mathbb{A}_{O_{K}}^{N} \rightarrow \mathbb{P}_{O_{K}}^{N}$ defined by $z_{0} \neq 0$. Both $x$ and $B$ are contained in $\mathbb{A}_{O_{K}}^{N}$. Since $X_{O_{K}}$ is smooth at $x$, we know that there are polynomial functions $G_{j} \in O_{K}\left[z_{1}, \ldots, z_{N}\right], 1 \leqslant j \leqslant N-d$, such that

- $X$ is locally defined by the equations $G_{1}=\ldots=G_{N-d}=0$; in particular,

$$
\mathcal{V}=X_{K}(K) \cap B=\left\{z \in B: G_{i}(z)=0 \text { for all } i=1, \ldots, N-d\right\}
$$

- the rank of the matrix $\left(\partial_{z_{j}} \bar{G}_{i}(0)\right)_{i \leqslant N-d, j \leqslant N}$ is $N-d$, where $\bar{G}_{i}=G_{i}$ modulo $\mathbf{m}_{K} O_{K}\left[z_{1}, \ldots, z_{N}\right]$.

Permuting the coordinates $z_{1}, \ldots, z_{N}$, we may suppose that the determinant of the matrix $\left(\partial_{z_{j}} \bar{G}_{i}(0)\right)_{i, j \leqslant N-d}$ is different from zero in $\mathbf{F}$. Denote by $\pi: B \rightarrow\left(O_{K}\right)^{d}$ the projection $\left[1: z_{1}: \ldots: z_{N}\right] \mapsto\left(z_{1}, \ldots, z_{d}\right)$. By Hensel's lemma, there exists a unique analytic diffeomorphism $\varphi:\left(O_{K}\right)^{d} \rightarrow \mathcal{V}$ such that $G_{i}((z, \varphi(z)))=0$ for all $i \leqslant N-d$.

Let $f$ be a birational map in $\operatorname{Bir}\left(X_{O_{K}}\right)$ such that $x \notin B_{O_{K}, f}$ and $f(x)=x$. Then, $f$ fixes the set $\mathcal{V}$ of points $z \in X_{K}(K)$ such that $r(z)=x$, and the action of $f$ on $\mathcal{V}$ is conjugate, via $\varphi$, to an analytic diffeomorphism on the polydisk $\mathcal{U}$. This concludes the proof of Proposition 3.4. 


\section{References}

[1] Bass, H. \& Lubotzky, A., Automorphisms of groups and of schemes of finite type. Israel J. Math., 44 (1983), 1-22.

[2] Bass, H., Milnor, J. \& Serre, J. P., Solution of the congruence subgroup problem for $\mathrm{SL}_{n}(n \geqslant 3)$ and $\operatorname{Sp}_{2 n}(n \geqslant 2)$. Inst. Hautes Études Sci. Publ. Math., 33 (1967), 59-137.

[3] Bedford, E., Lyubich, M. \& Smillie, J., Polynomial diffeomorphisms of $\mathbf{C}^{2}$. IV. The measure of maximal entropy and laminar currents. Invent. Math., 112 (1993), 77-125.

[4] BekKa, B., De la Harpe, P. \& Valette, A., Kazhdan's property (T). New Mathematical Monographs, 11. Cambridge Univ. Press, Cambridge, 2008.

[5] Bekka, B. \& Olivier, B., On groups with property $\left(\mathrm{T}_{\ell_{p}}\right)$. J. Funct. Anal., 267 (2014), 643-659.

[6] Bell, J.P., A generalised Skolem-Mahler-Lech theorem for affine varieties. J. London Math. Soc., 73 (2006), 367-379.

[7] Bell, J.P., Ghioca, D. \& Tucker, T. J., The dynamical Mordell-Lang problem for étale maps. Amer. J. Math., 132 (2010), 1655-1675.

[8] Borel, A., Introduction aux Groupes Arithmétiques. Publications de l'Institut de Mathématique de l'Université de Strasbourg, XV. Actualités Scientifiques et Industrielles, No. 1341. Hermann, Paris, 1969.

[9] - Linear Algebraic Groups. Graduate Texts in Mathematics, 126. Springer, New York, 1991.

[10] Bourbaki, N., Lie Groups and Lie Algebras. Chapters 1-3. Elements of Mathematics (Berlin). Springer, Berlin-Heidelberg, 1998.

[11] Cantat, S., Version kählérienne d'une conjecture de Robert J. Zimmer. Ann. Sci. École Norm. Sup., 37 (2004), 759-768.

[12] - Invariant hypersurfaces in holomorphic dynamics. Math. Res. Lett., 17 (2010), 833-841.

[13] - Sur les groupes de transformations birationnelles des surfaces. Ann. of Math., 174 (2011), 299-340.

[14] - The Cremona group in two variables. European Congress of Mathematics, VI (2012), $211-225$.

[15] - Morphisms between Cremona groups, and characterization of rational varieties. Compos. Math., 150 (2014), 1107-1124.

[16] Cantat, S. \& Dolgachev, I., Rational surfaces with a large group of automorphisms. J. Amer. Math. Soc., 25 (2012), 863-905.

[17] Cantat, S. \& Lamy, S., Groupes d'automorphismes polynomiaux du plan. Geom. Dedicata, 123 (2006), 201-221.

[18] Cantat, S. \& Xie, J., Algebraic actions of discrete groups: the $p$-adic method (expanded version). Preprint, 2018. Available at http://perso.univ-rennes1.fr/serge.cantat/ publications.html.

[19] Cantat, S. \& Zeghib, A., Holomorphic actions, Kummer examples, and Zimmer program. Ann. Sci. Éc. Norm. Supér., 45 (2012), 447-489.

[20] Caprace, P. E. \& RÉmy, B., Simplicity and superrigidity of twin building lattices. Invent. Math., 176 (2009), 169-221.

[21] Cornulier, Y., Sofic profile and computability of Cremona groups. Michigan Math. J., 62 (2013), 823-841.

[22] — Irreducible lattices, invariant means, and commensurating actions. Math. Z., 279 (2015), $1-26$.

[23] Cossec, F. R. \& Dolgachev, I. V., Enriques Surfaces. I. Progress in Mathematics, 76. Birkhäuser, Boston, MA, 1989. 
[24] Coulhon, T. \& Saloff-Coste, L., Isopérimétrie pour les groupes et les variétés. Rev. Mat. Iberoamericana, 9 (1993), 293-314.

[25] Deligne, P., Extensions centrales non résiduellement finies de groupes arithmétiques. $C$. R. Acad. Sci. Paris Sér. A-B, 287 (1978), A203-A208.

[26] DÉserti, J., Groupe de Cremona et dynamique complexe: une approche de la conjecture de Zimmer. Int. Math. Res. Not., 2006 (2006), Art. ID 71701, 27 pp.

[27] — Sur le groupe des automorphismes polynomiaux du plan affine. J. Algebra, 297 (2006), $584-599$.

[28] Dixon, J.D., Du Sautoy, M.P.F., Mann, A. \& Segal, D., Analytic Pro-p Groups. Cambridge Studies in Advanced Mathematics, 61. Cambridge Univ. Press, Cambridge, 1999.

[29] Epstein, D. B. A. \& Thurston, W. P., Transformation groups and natural bundles. Proc. London Math. Soc., 38 (1979), 219-236.

[30] Ershov, M., Golod-Shafarevich groups with property $(T)$ and Kac-Moody groups. Duke Math. J., 145 (2008), 309-339.

[31] Farb, B. \& Margalit, D., A Primer on Mapping Class Groups. Princeton Mathematical Series, 49. Princeton Univ. Press, Princeton, NJ, 2012.

[32] Fisher, D., Groups acting on manifolds: around the Zimmer program, in Geometry, Rigidity, and Group Actions, Chicago Lectures in Math., pp. 72-157. Univ. Chicago Press, Chicago, IL, 2011.

[33] Formanek, E. \& Procesi, C., The automorphism group of a free group is not linear. J. Algebra, 149 (1992), 494-499.

[34] Friedland, S. \& Milnor, J., Dynamical properties of plane polynomial automorphisms. Ergodic Theory Dynam. Systems, 9 (1989), 67-99.

[35] Glasner, Y. \& Monod, N., Amenable actions, free products and a fixed point property. Bull. Lond. Math. Soc., 39 (2007), 138-150.

[36] Greenberg, L., Maximal groups and signatures, in Discontinuous Groups and Riemann Surfaces (Univ. Maryland, College Park, MD, 1973), Ann. of Math. Studies, 79, pp. 207-226. Princeton Univ. Press, Princeton, NJ, 1974.

[37] Grothendieck, A., Éléments de géométrie algébrique. IV. Étude locale des schémas et des morphismes de schémas IV. Inst. Hautes Études Sci. Publ. Math., 32 (1967), 361.

[38] Grothendieck, A. \& Dieudonné, J.A., Eléments de Géométrie Algébrique. I. Grundlehren der Mathematischen Wissenschaften, 166. Springer, Berlin-Heidelberg, 1971.

[39] de la Harpe, P., Topics in Geometric Group Theory. Chicago Lectures in Mathematics. Univ. of Chicago Press, Chicago, IL, 2000.

[40] de la Harpe, P. \& Valette, A., La propriété $(T)$ de Kazhdan pour les groupes localement compacts. Astérisque, 175 (1989), 158 pp.

[41] Hrushovski, E., The elementary theory of the Frobenius automorphism. Preprint, 2004. arXiv:math/0406514 [math.LO].

[42] Ivanov, N. V., Subgroups of Teichmüller Modular Groups. Translations of Mathematical Monographs, 115. Amer. Math. Soc., Providence, RI, 1992.

[43] Kneser, M., Normalteiler ganzzahliger Spingruppen. J. Reine Angew. Math., 311/312 (1979), 191-214.

[44] Koblitz, N., p-adic Numbers, p-adic Analysis, and Zeta-Functions. Graduate Texts in Mathematics, 58. Springer-Verlag, New York, 1984.

[45] Kraft, H. \& Stampfli, I., On automorphisms of the affine Cremona group. Ann. Inst. Fourier (Grenoble), 63 (2013), 1137-1148. 
[46] Lang, S. \& Weil, A., Number of points of varieties in finite fields. Amer. J. Math., 76 (1954), 819-827.

[47] Lazard, M., Groupes analytiques p-adiques. Inst. Hautes Études Sci. Publ. Math., (1965), 389-603.

[48] LECH, C., A note on recurring series. Ark. Mat., 2 (1953), 417-421.

[49] Lubotzky, A., Discrete Groups, Expanding Graphs and Invariant Measures. Progress in Mathematics, 125. Birkhäuser, Basel, 1994.

[50] Macbeath, A. M. \& Singerman, D., Spaces of subgroups and Teichmüller space. Proc. London Math. Soc., 31 (1975), 211-256.

[51] Margulis, G. A., Discrete Subgroups of Semisimple Lie Groups. Ergebnisse der Mathematik und ihrer Grenzgebiete, 17. Springer-Verlag, Berlin, 1991.

[52] MCCARThy, J., Normalizers and centralizers of pseudo-Anosov mapping classes. Preprint, 1982. Revised version, 1994. Available at http://users.math.msu.edu/ users/mccarthy/publications/normcent.pdf.

[53] Millson, J. J., Real vector bundles with discrete structure group. Topology, 18 (1979), 83-89.

[54] Platonov, V. \& Rapinchuk, A., Algebraic Groups and Number Theory. Pure and Applied Mathematics, 139. Academic Press, Boston, MA, 1994.

[55] Poonen, B., p-adic interpolation of iterates. Bull. Lond. Math. Soc., 46 (2014), 525-527.

[56] Raghunathan, M.S., Torsion in cocompact lattices in coverings of $\operatorname{Spin}(2, n)$. Math. Ann., 266 (1984), 403-419. Correction in Math. Ann., 303 (1995), 575-578.

[57] - The congruence subgroup problem, in Proceedings of the Hyderabad Conference on Algebraic Groups (Hyderabad, 1989), pp. 465-494. Manoj Prakashan, Madras, 1991.

[58] Rapinchuk, A. S., Strong approximation for algebraic groups, in Thin Groups and Superstrong Approximation, Math. Sci. Res. Inst. Publ., 61, pp. 269-298. Cambridge Univ. Press, Cambridge, 2014.

[59] Robert, A.M., A Course in p-adic Analysis. Graduate Texts in Mathematics, 198. Springer-Verlag, New York, 2000.

[60] Serre, J. P., Lie Algebras and Lie Groups. Lecture Notes in Mathematics, 1500. SpringerVerlag, Berlin, 2006.

[61] Steinberg, R., Lectures on Chevalley Groups. Yale Univ., New Haven, CT, 1968.

[62] — Some consequences of the elementary relations in $\mathrm{SL}_{n}$, in Finite Groups - Coming of Age (Montreal, QC, 1982), Contemp. Math., 45, pp. 335-350. Amer. Math. Soc., Providence, RI, 1985.

[63] Stuck, G., Low-dimensional actions of semisimple groups. Israel J. Math., 76 (1991), $27-71$.

[64] Tomanov, G., On the congruence-subgroup problem for some anisotropic algebraic groups over number fields. J. Reine Angew. Math., 402 (1989), 138-152.

[65] Vinberg, È. B. (ED.), Lie Groups and Lie Algebras, III. Encyclopaedia of Mathematical Sciences, 41. Springer, Berlin-Heidelberg, 1994.

[66] WeIL, A., On algebraic groups of transformations. Amer. J. Math., 77 (1955), 355-391.

[67] Zimmer, R. J., Actions of semisimple groups and discrete subgroups, in Proceedings of the International Congress of Mathematicians, Vol. 1, 2 (Berkeley, CA, 1986), pp. 12471258. Amer. Math. Soc., Providence, RI, 1987.

[68] - Lattices in semisimple groups and invariant geometric structures on compact manifolds, in Discrete Groups in Geometry and Analysis (New Haven, CT, 1984), Progr. Math., 67, pp. 152-210. Birkhäuser, Boston, MA, 1987. 


\author{
Serge Cantat \\ Institut de recherche mathématique de Rennes \\ Université de Rennes 1 \\ Beaulieu - Bâtiment 22-23 \\ 263, avenue du Général Leclerc \\ FR-35042 Rennes Cedex \\ France \\ serge.cantat@univ-rennes1.fr \\ JunYI XIE \\ Institut de recherche mathématique de Rennes \\ Université de Rennes 1 \\ Beaulieu - Bâtiment 22-23 \\ 263, avenue du Général Leclerc \\ FR-35042 Rennes Cedex \\ France \\ junyi.xie@univ-rennes1.fr
}

Received July 7, 2015

Received in revised form February 3, 2018 